\title{
UNITED AIRCRAFT. CORPORATION
}

RESEARCH LABORATORIES

East Hartford, Connecticut

\author{
UARL Report R953100-22 \\ Semiannual Report \\ Under Contract AT(11-1)-2277 \\ August 1, 1974 \\ through \\ January 31, 1975
}

Project Title: High- $\beta$ Capture and Mirror Confinement of Laser Produced. Plasmas Name of Contractor: United Aircraft Corporation Research Laboratories

Reported by:

Oean Dethent

A. F. Haught

Principal Investigator

\section{NOTICE}

This report was prepared as an account of work This report was prepared States Government. Neither sponsored by the United States United States Energy the United States nor the United States nor any of Research and Development Administration, nor any of their employees, nor any of their contractors, subcontractors, or their employees, makes any warranty, express or implied, or assumes any legal liability or responsibility for the accuracy, completeness or usefulness of any information, apparatus, product or

process disclosed, or represents
infringe privately owned rights.
Date: January 31, 1975

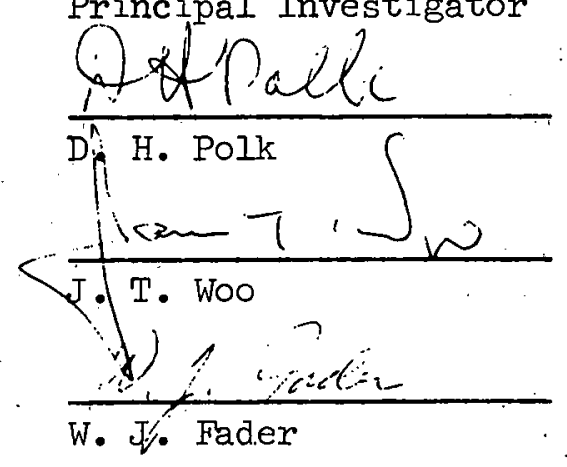

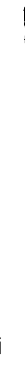

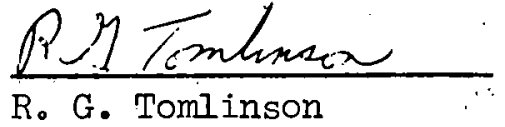

R. G. Tomlinson

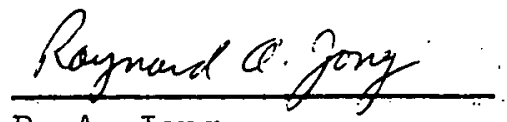

R. A. Jong

is is lece

W. B. Ard.

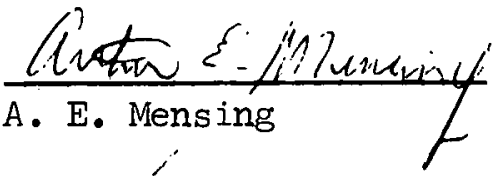

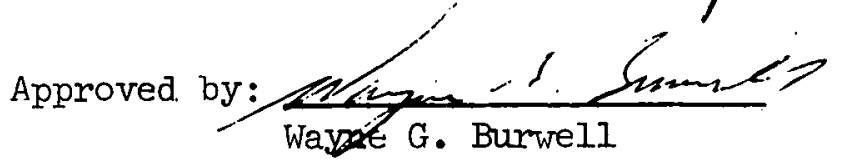

Manager of Energy Research

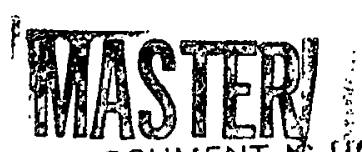




\section{DISCLAIMER}

This report was prepared as an account of work sponsored by an agency of the United States Government. Neither the United States Government nor any agency Thereof, nor any of their employees, makes any warranty, express or implied, or assumes any legal liability or responsibility for the accuracy, completeness, or usefulness of any information, apparatus, product, or process disclosed, or represents that its use would not infringe privately owned rights. Reference herein to any specific commercial product, process, or service by trade name, trademark, manufacturer, or otherwise does not necessarily constitute or imply its endorsement, recommendation, or favoring by the United States Government or any agency thereof. The views and opinions of authors expressed herein do not necessarily state or reflect those of the United States Government or any agency thereof. 


\section{DISCLAIMER}

Portions of this document may be illegible in electronic image products. Images are produced from the best available original document. 
Semiannual Report Under Contract AT(11-1)-2277

For the period August 1, 1974 through January 31, 1975

High- $\beta$ Capture and Mirror Confinement

of Laser Produced Plasmas

TABLE OF CONTENTS

$\underline{\text { Page }}$

I. SUMMARY ......................... 1

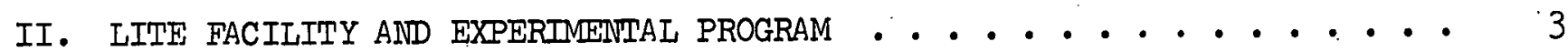

A. LITE Experiment Chamber Facility . . . . . . . . . . . 3

1. Experiment Chamber . . . . . . . . . . . . . . . 3

2. Magnet . . . . . . . . . . . . . . . . . 4

3. Laser System ..................... . . 5

4. Particle Suspension System . . . . . . . . . . . 6

B. Plasma Production Experiments . . . . . . . . . . . . 10

C. LITE Neutral Beam Line Development . . . . . . . . . . . 12

1. Ion Source Tests . . . . . . . . . . . . . . 12

2. Complete Beam Line . . . . . . . . . . . . . 15

a. Beam Line Assembly ................ . . 15

b. Beam Line Tests ................... 16

3. Future Test Program . . . . . . . . . . . . 17

D. Diagnostics . . . . . . . . . . . . . . . 17

1. Fast Atom Energy Analyser . . . . . . . . . . . . . 18

2. Heavy Ion Probe . . . . . . . . . . . . . . 19

3. Microwave Interferometer . . . . . . . . . . . . 20

4. Data Handling and Reduction . . . . . . . . . . . 20

5. Analysis of Plasma Streak Photographs . . . . . . . . . 21

III. FOKKER-PLANCK AND BUILDUP SIMULATION CALCULATIONS • • • • • • • • • 23

A. Fokker-Planck Calculations of LITE Plasma Development. . . . . . 23

1. Modifications to ISOTIONS Code . . . . . . . . . . • 23

a. Iteration Subroutine .............. 23

b. Charge Exchange with Background Neutrals . . . . . . 24

c. Neutral Injection . . . . . . . . . . . . 25

d. Rate Coefficients ................ 26 
TABLE OF CONTENTS Cont.

2. Fokker-Planck Results . . . . . . . . . . . $\frac{\text { Page }}{26}$

a. Target Plasma Decay and. Injection Buildup in LITE . . . . 26

b. Delayed Injection ............... 29

c. Distribution Functions . . . . . . . . . . . 29

d. Initial Conditions of the Mirror Confined Plasma . . . . . 31

B. Target Buildup Simulation . . . . . . . . . . . 34

1. Simulation Model ......................... 34

2. Results of Simulation ............... 39

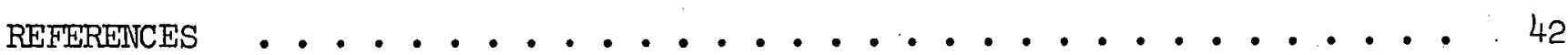

IIST OF FTGURES . . . . . . . . . . . . . . . . . . . 44 
UARL Report R953100-22

Semiannual Report Under Contract AT(11-1)-2277

For the period August 1, 1974 through January 31, 1975

High- $\beta$ Capture and Mirror Confinement

of Laser Produced Plasmas

I. SUMMARY

The Laser Initiated Target Experiment (LITE) at the United Aircraft Research Laboratories is investigating the use of a dense, mirror confined, laser produced plasma as the target for an energetic neutral injection beam to create and sustain a high temperature, high density, mirror confined fusion plasma. For these investigations, the target plasma is produced by laser irradiation of a solid particle of lithium hydride electrically suspended in vacuum within the confinement field. Experimental studies of the target plasma formation have shown that fully ionized, spherically symmetric plasmas containing $10^{16}-10^{17}$ ions and electrons with average energies up to $500-800 \mathrm{eV}$ can be produced by irradiation of $40-80 \mu$ dia lithium hydride particles with $\sim 20$ Joule, I nsec rise, 5 nsec duration laser pulses. Upon expansion within a $15 \mathrm{kG}$ center field, mirror ratio and well depth $=2,32 \mathrm{~cm}$ long minimum-B magnetic field, the resulting plasma is observed to fill the containment volume and is captured and confined at a density above $10^{13} \mathrm{~cm}^{-3}$ within a $400 \mathrm{~cm}^{3}$ tetrahedral volume, $\sim 6 \mathrm{~cm}$ indiameter and extending $\sim 20 \mathrm{~cm}$ along the mirror axis. Fokker-Planck calculations have been carried out examining the evolution of the confined laser produced plasma upon interaction with a $10 \mathrm{keV}$ energetic neutral beam. Charge exchange collisions between the plasma ions and beam neutrals serve to heat the confined plasma to the beam energy, and ionization of the beam neutrals acts to sustain the confined plasma against the losses due to coulomb decay or charge exchange with background neutrals. From the calculations, a $10 \mathrm{keV}, 1.0 \mathrm{~A}$ equivalent neutral beam incident on the $400 \mathrm{~cm}^{3}$ confined laser produced target plasma will result in the formation of a $10^{12} \mathrm{~cm}^{-3}$, $10 \mathrm{keV}$, injection sustained mirror plasma. The primary objective of the UARL Laser Initiated Target Experiment is the investigation of this injection sustained steady state. In addition, studies are being conducted under this program to examine the application of the laser plasma production technique to larger particles and higher energies and to investigate the mechanisms whereby the expanding, laser produced plasma is captured and upon mirror confinement fills the containment region. The objective of these studies is to develop the basis for extending the laser produced target plasma approach to the larger plasma systems of future research and reactor interest. 
During the current report period, primary attention has been placed on the construction of the LITE apparatus. The LITE plasma production and confinement system has been developed from an assemblage of component parts to a tested, operating, experimental facility. The feedback particle suspension system has been incorporated in the confinement chamber, debugged, and refined and is in routine use, suspending particles of up to $100 \mathrm{~m}$ diameter at the chamber center with a position jitter as small as $\sim 20 \mu \mathrm{m}$. Modifications have been made to improve the reproducibility of the laser pulse output, and the system now employs two direction laser irradiation

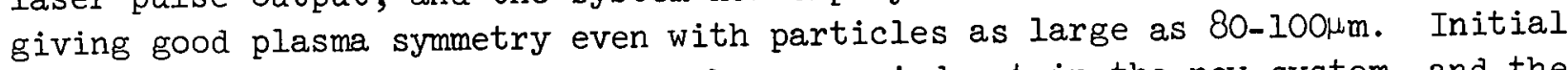
plasma production experiments have been carried out in the new system, and the prepulse and heating conditions established for the generation of $400-600 \mathrm{eV}$ symmetric plasmas with greatly increased reproducibility. Diagnostic instrumentation used for these initial experiments involve charge collector probes, with the data recorded and analyzed by the on-line PDP-ll computer facility for plasma symmetry, total ionization, and energy. Additional diagnostics, including an electrostatic energy analyzer, microwave interferometer, and the heavy ion probe, under development in a cooperative program by Hickok et al at RPI, are being prepared for installation in the experiment chamber for use in the target plasma confinement investigations scheduled for the first part of the next report period. In a parallel effort, studies of the operating characteristics of the MATS III ion source obtained from the Lawrence Livermore Laboratory have been carried out, and the neutralizer, ion deflector, drift tube, and termination tank beam line components have been assembled as a neutral beam line test stand. The vapor spray heads for the neutralizer have been constructed and are ready for installation for beam neutralization investigations. As presently scheduled, the neutral beam development effort is being conducted to produce a $1 \mathrm{~A}, 10 \mathrm{keV}$ neutral hydrogen beam line for incorporation into the LITE facility in March for initial injection experiments during the later half of the next report period. These experiment chamber, plasma production, diagnostic instrumentation, and beam development results are discussed in Sections II of this report. Theoretical investigations during this report period have included further development of the multi-species Fokker-Planck code, calculations of the LITE plasma behavior using this code, and a simulation analysis of the plasma buildup. The ISOTIONS multi-species Fokker-Planck code developed at the Lawrence Livermore Laboratory was modified to eliminate certain numerical instabilities in the iteration scheme, to include the effects of background neutrals and wall reflux on the plasma decay, to incorporate terms modeling the plasma buildup by neutral injection, and to use energy dependent cross-sections averaged over the distribution function. Calculations with the modified code have been performed to determine the effects of the lithium component on the plasma decay, the impact of delayed neutral injection, and the possibility of instabilities occurring during the injection heating phase of the plasma development. A simulation analysis of the plasma buildup has been undertaken to examine the erosion or growth of the plasma boundary through the combined processes of ionization and charge exchange collisions with the beam and interaction with the background neutrals. A diffusive particle transport mechanism associated with the finite ion Larmor orbit size and important at beam energies below $40 \mathrm{keV}$ has been identified through this analysis. These theoretical studies are reported in section III. 


\section{LITE FACIIITY AND EXPERTMENTIAL PROGRAM}

The LITE experimental system, described in detail in Ref. 1 , consists of a neutral beam source, experiment chamber, and beam termination tank, the irradiating laser, and a common, rigid base which is supported on pneumatic vibration isolators. During this report period, the LITE system components have been received, leak checked. and surface finished for ultra high vacuum, and assembled and operated in two parts: the laser and experiment chamber mounted on the support base for plasma production and confinement studies, and the neutral beam source and beam termination tank set up separately for testing of the ion source and beam line components. Assembly and. operation of the experiment chamber and its essential subsystems is described in Section II-A, and two-beam plasma production experiments with this system using feedback particle suspension are presented in Section II-B. In section II-C, beam extraction tests with the MATS III ion source are described along with assembly of the beam line components into a complete neutral beam test facility. Additional diagnostics under development for the LITE experiment and operation of the computer based data handling system are presented in section II-D.

\section{A. LITE Experiment Chamber Facility}

\section{Experiment Chamber}

The experiment chamber, the central component of the LITE facility, serves both as a mounting reference for the confinement magnet, diagnostic instrumentation, and neutral beam line and as a vacuum pumping system to meet the experimental requirement that mirror confined plasma losses by charge exchange with background neutrals be comparable with or smaller than losses by Coulomb scattering. Background neutrals arise from residual gas in the vacuum chamber, from wall bombardment by plasma ion escaping through the magnet mirrors, and, in the case of neutral injection, directly from charge exchange and indirectly by collision with the walls of these fast, randomly directed atoms. Hence, the vacuum chamber must not only provide the necessary initial and. steady state vacuum but also must be of sufficient volume to geometrically attenuate the large transient wall reflux generated by the initial target plasma decay and, with neutral injection, by the initial charge exchange process. To satisfy these requirements, the experiment chamber, show in Figs. 1-4, is built in the form of a vertical cylinder $\sim 120 \mathrm{~cm}$ diameter by $\sim 240 \mathrm{~cm}$ long divided into three sections of approximately equal length. The center section, supported. from the main base and having 48 access ports, is the experiment region in which are mounted the baseball seam magnet (magnetic axis vertical), irradiating optics, diagnostic apparatus and essentially all of the auxiliary systems. The two end chambers, supported directly from the center section for ease of disassembly, increase the chamber volume to $\sim 2 \times 10^{6} \mathrm{~cm}^{3}$ and incorporate liquid nitrogen dewars which provide $>10^{5} \mathrm{~cm}^{2}$ of cryogenic, titanium-coated getter surface for direct pumping of background neutrals. For the initial plasma production and target plasma capture and confinement experiments, a chamber vacuum of $\sim 10^{-7}$ Torr is more than adequate and diffusion pumping rather than dewared titanium gettering is being used 
for convenience. However, the liquid nitrogen dewars have been constructed and are prepared for installation within the experiment chamber to achieve the ultra high ( $3 \times 10^{-9}$ Torr) initial vacuum and sustained pumping required for the neutral injection experiments scheduled to be undertaken during the next report period.

After an initial assembly of the bare experiment chamber proved all the weld. seams and the copper $0-r i n g$ seals used on the large ( 50 in. diameter) main chamber flanges to be leak tight, the chamber was disassembled for final finishing of the intermal surfaces. Although the stainless steel plate from which the chamber is constructed was pre-sanded to a \#4 finish, a clean cut finish similar in structure to a $32 \mathrm{rms}$ lathe finish with a sharp tool, inevitably numerous small pits and. scratches resulted from the fabrication process. These surface defects and any pits or voids encountered in the welds were ground out, providing a clean, void free experiment chamber vacuum surface. While laborious, the surface finishing uncovered and eliminated a number of weld voids which through outgassing would have introduced significant difficulties in achieving ultra high vacuum operation and. may have presented problems even at the $\sim 10^{-7}$ Torr of the plasma production experiments. On the basis of this experience, both to ensure rapid pumpdown and to avoid the necessity for later disassembly and rework, all of the components introduced within the chamber have been designed for ultra high vacuum capability and have had their surfaces similarly treated prior to initial installation. A description of the major subsystems, their assembly on the experiment chamber, and of the operational results obtained to date are given in the following sections.

\section{Magnet}

The baseball seam magnet coil, developed for the IITE program and used for earlier laser plasma capture and. confinement investigations (see Refs. 1-3), is of spherical baseball geometry and provides a $15 \mathrm{kG}$ center field intensity, mirror ratio and well depth of 2 , with a distance between mirror points of $32 \mathrm{~cm}$. The magnet is mounted concentric with the experiment chamber on equatorial supports carried on four of the large (14.5 in. O.D.) flanges on the center chamber midplane (Figs. 2-6). Auxiliary flexible vacuum lines between the magnet vacuum shell and a set of chamber flanges enclose the magnet electrical and $\mathrm{LN}_{2}$ cooling leads, isolating them from the main vacuum chamber. Utilizing the greater space available within the new experiment chamber, the electrical and $\mathrm{LN}_{2}$ cooling isolation lines were improved over the original design used in the polyhedron chamber of the previous experiments, where space limitations led to a cumbersome, delicate, although workable, seal. In this installation, the magnet has been operated satisfactorily with full cooling to $\mathrm{LN}_{2}$ temperature and, using an auxiliary 200 Amp power source, to provide long duration, $<I \mathrm{kG}$ fields for initial beam alignment of the heavy ion probe being developed. for the LITE experiment by R. Hickok, J. Stufflebeam, et al. (Ref. 4,5) of Rensselaer Polytechnic Institute (see Section II-D).

The magnet power system has been modified to eliminate some operational limitations encountered in the earlier experiments. Powering of the magnet at full 
current (2050 Amps) for the maximum design duration of 1 sec was marginal because of the voltage limit of the substation transformer. In addition, for safety and to minimize the possibility of internal voltage breakdown, the magnet was designed to operate with one end of the coil grounded, which created conflicts with other large power users operating off the same transformer. Both problems have been eliminated by installation of a simple, inexpensive, air-cooled, isolation transformer specifically designed for this system with a $5 \mathrm{sec} / \mathrm{hr}$ duty cycle at maximum current. The transformer includes secondary taps providing $1: 1,1: 1.25$, and $1: 1.5$ voltage step-up ratios, thereby providing an increase in open-circuit d.c. voltage from 3400 to a maximum of 5100 volts. The modified power system, without the magnet, has been tested satisfactorily at low voltage and is now connected for full power magnet operation. Full power tests with the magnet, scheduled for the next $\mathrm{LN}_{2}$ magnet cooldown, will complete the preparation of the magnet for plasma capture and confinement experiments.

\section{Laser System}

The laser system, designed to provide two-direction irradiation of the target particle, consists of the laser, the beam transfer optics, the laser focusing lenses, the beam extraction optics, and the laser focal spot locater. The various components of the system, described below, may be seen in the photographs of Figs. 2 , 3,5 , and 6 .

As described in Ref. 1, a number of improvements in the laser have been incorporated to provide greater reliability and to improve the laser pulse parameters. One of the more intractable problems encountered in previous experiments was instability of the laser focal position resulting from thermal distortion of the laser table, introduced by the use of an aluminum laser base plate on a steel table, and changes in the relative position of the separately mounted laser table and experiment chamber. In the LITE facility, the laser has been mounted on an all-aluminum laser table to minimize thermal distortions, and both the laser table and experiment chamber as well as the beam transfer optics are mounted on a common, rigid, vibration-isolated. base. This new system, in tests to date, appears to have effectively eliminated the instability in beam focal position. In addition, new, lower voltage, higher hold-off ratio Pockel's cells with modified power systems for more uniform voltage were installed in the pulse-forming optical switches. Finally, higher-gain amplifier rods replaced those damaged in previous operations. The laser system with these improvements has been used for the plasma production experiments in the new facility as reported. in the section II-B, producing with a conservative amplifier pumping voltage of $\sim 85$ percent of maximum, an output pulse with very good repeatability at an energy of $\sim 30$ Joules, a risetime of $\lesssim 2.5 \mathrm{nsec}$, and a half-wid.th of $\sim 7 \mathrm{nsec}$.

As shown in Figs. 2 and 3 , the beam transfer optics for two-direction irradiation of the target particles consist of a mirror on the laser table at the output of the last stage amplifier followed by a 50 percent beam splitter located near the experiment chamber equidistant from the two final turning mirrors which are mourted on the experiment chamber and which direct the beams onto the focusing lenses. Since the final turning mirrors are equally spaced from their respective focal lenses, the 
two beams which irradiate the target particle from opposite sides travel equal path lengths and hit the particle simultaneously, reducing any unbalanced forces on the particle from vapor blowoff reaction.

The laser focusing lenses are $20 \mathrm{~cm}$ nominal focal length, plano-convex lenses of BK-7 glass. having a clear aperture of $4.5 \mathrm{~cm}$ diameter. As show in Figs. 5 and 6 , each lens is mounted in the inner end of a bellows-sealed tube so that the lens also comprises the vacuum window. By means of an external threaded sleeve, each lens may be moved axially to position the minimum laser beam focus on the target particle. Since the feedback particle suspension system provides considerable freedom in the choice of suspended particle position, it was possible to eliminate the bulky apparatus previously required for transverse adjustment of the laser focusing lens. Instead, the beam transfer optics are installed with the laser beam nominally coaxial with the lens, and the subsequent small transverse displacements of the beam required to position both foci at the same point are obtained by small angular adjustments of the final beam turning mirrors. As described in Ref. 1 , the axes of the focusing lens are at an angle of $137^{\circ}$ to prevent direct feedback into the laser. If the transmitted beam. were allowed to impinge on the chamber walls it could. produce a large gas efflux and, as a result, directly opposite each focusing lens is a fixed lens of $27 \mathrm{~cm}$ focal length (Figs. 5 and 6) which collects transmitted. radiation and directs it out of the chamber through a vacuum window and into an extermal Iight dump.

The location of the laser focal volume for each lens must be determined to within $\sim 50 \mu$, and is measured directly by placing a flat surface at the lens focus and examining the position of the burm spot produced by the irradiating laser beam. The mechanism shown in Figs. 5 and 6 is incorporated in the vacuum chamber for these measurements and allows locating and checking the focal spot location at full vacuum as part of the operating routine, without disturbing the experimental set-up. The device holds a supply of mylar tape which is stretched across an open fork in a manner that permits the two beams to be separately or simultaneously focused on opposite sides of the active area of the tape. By means of a 4-bar linkage actuated by a rotary vacuum feedthrough, the tape holder can be inserted from a park position well outside the magnet to the laser focus position at the magnet center. Using two auxiliary optical viewing systems of 40X magnification looking along the laser beam extraction axes (Fig. 2), reticle circles of $\sim 100 \mu$ diameter define the unique point in space at which the target particle must be suspended to be in the laser focal volume.

\section{Particle Suspension System}

The target particle suspension system includes the particle injector, the electron gun for electrically charging the particles, the suspension electrodes, the suspension voltage power source, and the vidissector particle detector/tracker system that provides the particle position sensing required for feedback-controlled suspension and positioning of the particle. 
The particle injector is a rotary vacuum feedthrough controlled, spring actuated piston that throws several particles up into the capture volume from a powder storage cup mounted below the magnet, as show in Fig. 6. The electron gun (Fig. 6), inserted from a park position outside the magnet by means of a 4-bar linkage device, irradiates the particle with an electron beam of 50-100 V energy, and produces, by secondary emission, a positively charged particle which can then be captured by the electric fields produced by the suspension electrodes. The suspension electrodes, unchanged from those used in previous experiments, are plates covering the interior surfaces of the magnet, contoured to fit the magnet surfaces so that the electrodes introduce no significant constriction of the plasma confinement volume. Functionally, the electrodes produce essentially the same field. patterm as a cubical electrode array - a rotating electric field in the three-phase a.c. operational mode or three mutually perpendicular d.c. fields in the feedback controlled mode.

The feedback controlled particle suspension system comprised of the suspension voltage source and particle detector/tracker system is operational and is being used in plasma production experiments. In the LITE system, electric fields are used to capture, hold, and position a charged, isolated, lithium hydride particle in vacuum in the center of the magnetic confinement field at the focus of the irradiating laser beam. In past experiments, particle suspension has been accomplished by a passive system of superimposed. d.c. and three phase a.c. fields which required the use of a low pressure ( $5 \times 10^{-4}$ Torr) background gas to damp the excess kinetic energy of the captured particle. Although this gas was pumped out prior to plasma production by laser heating of the particle, such operation is incompatible with the ultra high vacuum and surface gettering requirements of a neutral beam sustained target plasma. As a result, a radically different, active feedback controlled suspension system has been developed for LITE. The design of the system and the details of its components are discussed in Ref. 1. In the feedback suspensjon system, the voltages applied to the suspension electrodes are derived from the position and. velocity of the particle, providing both a reference center and electronic damping so that the particle is precisely positioned at high vacuum within the focal region of the high power laser beam.

A 40-100 $\mu \mathrm{m}$ diameter lithium hydride particle from which the plasma is generated. is charged by an electron gun and mechanically projected toward the center of the evacuated experiment chamber. The position of the particle in space is observed. electro-optically by three image dissector photomultipliers (vidissectors) which produce output voltages proportional to particle image location on the tube photocathode. The resulting particle position signals, electronically transformed. to the coordinate frame of the suspension electrodes, are fed to a lead/lag circuit in the control network which produces and sums voltages proportional to the particle position and velocity. The dynamic control voltages thereby generated are then increased in six wide-band, bi-polar, high voltage amplifiers and fed to the six suspension electrodes producing up to $\pm 5 \mathrm{kV}$ on any plate. Provision is made in the system for switching to passive a.c. operation by driving the high voltage 
amplifiers from a three phase oscillator. Such operation was incorporated to facilitate initial alignment and debugging of. the system and to separate the problems of particle capture from those of particle confinement.

Successful feedback control of a suspended particle was first achieved in October, 1974. In these and subsequent tests, particle capture was effected using the three phase, a.c. operating mode. Following localization of the particle orbit within the field of view of the vidissector detector/trackers, the feedback loop was closed, switching the suspension system from the a.c. mode to active feedback operation. The position voltages from the three detector/trackers during the closing of the feedback loop on an orbiting particle are show in Fig. 7. The particle, whose trajectory in the capture mode was a $17 \mathrm{~Hz}$ circular orbit slightly greater than one millimeter in diameter, was clamped at the position defined by the detector/trackers in less than $100 \mathrm{msec}$ following closure of the feedback loop. Oscilloscope pictures made using two of the position voltages to produce $x-y$ plots are show in Fig. 8. The exposure in Fig. 8 was $1 / 4 \mathrm{sec}$, sufficiently long to display nearly four full orbits of the a.c. suspended particle and reveals a jitter comparable to the one millimeter diameter particle orbit. The particle jitter, after closure of the feedback loop, is too small to be seen in Fig. 8. The fluctuations of the feedback controlled particle about its central position are observed. in the position signals displayed at high gain in Fig. 9. The rms value of these fluctuations was noted to vary significantly from particle to particle, being only a few microns rms in the best case and somewhat larger than 100 microns in the worst case.

The suspended particles are illuminated by an argon-ion laser beam at an intensity of 20 watts $/ \mathrm{cm}^{2}$. The measured signal levels for a $60 \mu \mathrm{m}$ diameter particle correspond to $\sim 50$ nanowatts in the optical image at the vidissector photocathodes in good agreement with the image power calculated assuming an effective particle reflectivity of ten percent. The measured signal level is well above the one nanowatt minimum power level which bench tests with the vidissectors had established as the threshold for achieving usable signal to noise ratio outputs. Position signals are generated by scanning the vidissector photocathode at $50 \mathrm{kHz}$. If the vidissector sees a signal greater than a value pre-set in a comparator, it triggers a multivibrator circuit producing a fixed amplitude, fixed width pulse (Fig. 10a). The train of pulses generated as the scan sweeps back and forth across the image is Fourier analysed to produce a position discriminant. For a comparator setting which is too low, photocathode noise can cause the generation of extraneous pulses, introducing jitter in the derived particle position. For a comparator setting which is too high, variations in the optical signal amplitude result in "dropouts" from the pulse train where the multivibrator is not triggered, again producing jitter in the electronically sensed particle position. The comparator was initially pre-set at the one nanowatt minimum usable signal level; however, the background level was found. to be much higher, approximately 10 percent of the $\sim 50 \mathrm{nW}$ peak signal, apparently as a result either of wall reflections of particle scattered light or of internal

scattering within the detection optics. Even with the comparator threshold. set well above the noise background and sufficiently below the peak signal so that no 
significant number of "dropouts" occurred, some particles still exhibited considerable jitter in position. The lithium hydride particles are seen under high magnification to exhibit a wide variety of shapes, some of which are quite irregular. This irregularity leads to a signal amplitude modulation (Fig. 10b) which alters the point at which the multivibrator is fired (Fig. 10c) and, as a result, shifts the relative position of the pulses in the train. As a consequence the particle exhibits a signal-amplitude-induced jitter. For each given particle size and. geometry there is a comparator setting which gives maximum particle stability. The. need to vary the comparator setting for different geometry particles was not anticipated in the original design of the feedback controlled suspension system and no ready access to the comparator threshold setting was provided. A circuit modification is now being incorporated into the signal processing electronics to detect the peak and average vidissector signals and to adjust the comparator threshold automatically to compensate for changing background levels. Since a low threshold. setting is desired to facilitate operation over the widest range of conditions during particle capture and a high threshold setting is necessary for particle confinement with maximum particle stability, the comparator threshold relative to the peak to background ratio will be variable by means of a front mounted manual control.

As a consequence of the small latitude between the high background level and. the modulated signal amplitude, the inflexible comparator threshold setting initially provided has prevented particle control over a large spatial volume. The total signal level decreases as the particle moves away from the center of the chamber due to a combination of image defocusing and decreasing particle illumination. Since the comparator threshold had to be pre-set above the background noise with the particle at the center position, only a few millimeters displacement of the particle could be tolerated before the general decrease in signal intensity produced excessive "dropouts", and particle control was lost. With this small "box size" over which particle control was possible, only particles entering the box with very low velocities could be decelerated and captured within the box by the suspension fields, and the current particle injection and charging does not incorporate this degree of precision in introducing particles. However, the automatic electronic adjustment of the comparator threshold should permit operation over a greatly increased "box size"; and a second circuit modification, to ensure positive control, will delay application of the control voltages until the particle is well within the field of view of all three vidissectors. These modifications are anticipated to allow particle capture with no requirement for more accurate particle injection, and additional work will be undertaken during the next contract period on feedback controlled particle capture as well as confinement. Such operation will decrease the experiment cycle time during the target plasma development phase and will be required for ultra high vacuum operation during the neutral injection heating experiments. In its current operating mode, the feedback controlled suspension system has eliminated uncertainty and variability in the positioning of the particle in the laser focal region, greatly improving the reproducibility of the plasma production and making possible the rapid. progress of the target plasma development, reported in the next section. 


\section{B. Plasma Production Experiments}

Plasma production experiments are currently in progress to determine the optical and laser parameters required to generate symmetric, fully ionized, energetic plasmas and to evaluate the reproducibility obtained by two-direction laser irradiation of LiH particles suspended in the laser focal volume by the feedback controlled suspension system (Section II-A). Since no magnetic fields are used in these tests, the plasma expand.s freely, and the experiment chamber vacuum need only be sufficient to yield. plasma ion and electron mean free paths that are large compared to the dimensions of the chamber. Hence, the chamber vacuum in these experiments is $\sim 10^{-6}$ Torr, limited by temporary Plexiglass windows and rubber gasket flange seals used to provide maximum visual access and easy access for rapid adjustments and modifications. The plasma diagnostic being used in these initial tests is an array of apertured. ion charge collectors (Ref. 6) of $1 \mathrm{~cm}$ diameter aperture located at $50 \mathrm{~cm}$ from the plasma origin, giving a collector aperture solid angle of $8 \pi \times 10^{-5}$ steradian. With the charge collectors at a distance of $50 \mathrm{~cm}$, the peak ion currents for plasmas generated from 60-80 $\mu \mathrm{m}$ diameter $\mathrm{LiH}$ particles are of the order of $100 \mathrm{~mA}$ or less, and the expansion times of the plasma from origin to collector is of the order of microsecond.s. Thus, a measurement of the time-resolved ion current on the collector plate, using the laser pulse to provide the zero time reference, provides the data required to infer both plasma quantity and energy. To determine symmetry, six collectors are used: (1) two collectors (East and West) viewing plasma expanding nearly (within 20 degrees) along the direction of the two laser beams and (2) four collectors (North, South, Top, and Bottom) viewing plasma expanding nearly normal to the laser beams.

The results of previous experiments showed that for one-direction irradiation with laser pulses of 1-2 nanosecond risetime, it was not possible to fully vaporize and ionize particles larger than $\sim 40$ m diameter unless a low power prepulse was used to initially vaporize the particle. For larger particles, the total ionization was low and little, if any, plasma could be observed on the shadowed side of the particle. Using a long duration (20-40 nsec) low power ( $10 \mathrm{MW}$ peak) prepulse resulted in increased ionization and improved symmetry, but, especially for larger particles, substantial improvements in ionization and symmetry were obtained only by using so much prepulse that the plasma energy was greatly reduced. Both measurement and visual observations indicated that one-direction irradiation resulted in an unvaporized portion of the particle being prematurely forced out of the laser focal volume by the reaction to unbalanced vapor expansion forces on the irradiated. side. These results were the basis for the incorporation of two-direction irradiation in the new experiment chamber.

From the initial plasma production experiments using the new experiment facility, these effects are clearly apparent. As shown in Figs. 11 and 12, irradiation of $\sim 60 \mu \mathrm{m}$ diameter particles with a single $2.5 \mathrm{nsec}$ risetime laser pulse yield.s low total ionization, and little plasma is observed on the shadowed side of the particle. With two-direction irradiation (Fig. 13), the quantity of plasma is increased, and. 
the distribution is more nearly spherical. However, since no prepulse was used. for the cases shown, the total quantity of plasma is still somewhat less than expected for an $\sim 60 \mu \mathrm{m}$ diameter spherical particle, and the quantity of plasma expanding back into the laser beams (East and West) is greater than that expanding in the transverse directions. It appears from these results, therefore, that even with two direction irradiation a laser prepulse to vaporize the particle and. partially expand the vapor is necessary to achieve full ionization and symetry.

The effects of a prepulse on the properties of the plasma produced by irradiating particles are more highly specific and less readily generalized than in the case of massive targets (Ref. 7). Since the functional dependence of these effects on parameters such as the ratio of particle size to focal spot size, the laser intensity distribution, and the laser pulse time history, etc. are not quantitatively known, they mist be empirically determined for each experimental situation. In the current experiments, the prepulse has a risetime of $20-30 \mathrm{nsec}$ to maximum amplitude just prior to the start of the high power pulse, and, as the amplitude of the prepulse is increased, the degree of ionization is increased, the energy distribution is broadened, and the average energy of the plasma is decreased slightly. This is show by comparison of the data of Fig. 13 for two beams with no prepulse and Fig. 14 for two beams with peak prepulse intensity estimated to be $\lesssim 5$ percent of the main pulse intensity.

The variation of average plasma energy $\left(\bar{\epsilon}=E /\left(N_{e}+N_{i}\right)\right.$, where $E$ is total plasma energy, $N_{e}$ and $N_{i}$ are the total number of electrons and ions -- both determined. from the charge collector data of plasma current as a function of time) with prepulse intensity is shown in Fig. 15. Because absolute measurements of prepulse amplitude have not yet been made, the amplitude is given in terms of the controlling experimental variable which is the angular rotation of the Glan-Laser polarizer prism at the output end of the optical switch used to shape the pulse risetime. However, the oscilloscope records of the pulse amplitude-time history indicate a maximum prepulse amplitude of $<5$ percent of the main pulse amplitude corresponding to the maximum rotation angle of $\approx 22$ degrees. These initial results have been very reproducible, and, for prepulse amplitudes obtained at rotation angles $>10$ degrees, the plasmas have been spherically symmetric in density within \pm 25 percent and in energy within \pm 20 percent.

With no prepulse, observed. average plasma energies of $\sim 600 \mathrm{eV}$ (Fig. 15) are obtained. With 20-30 Joule (10-15 Joules/beam), $\sim 2.5$ nsec risetime, $\sim 7$ nsec duration laser pulses, although, as noted above, particle ionization appeared to be incomplete and the plasma expansion was asymmetric. For these laser parameters, the similarity model of the plasma heating process (Ref. 6), which assumes an initially fully ionized, cold plasma that absorbs laser energy uniformly, predicts an average plasma energy of 600-700 eV. This agreement in energy would be expected because, as shown in the calculations of the time history of the plasma heating process, essentially all the laser energy absorption takes place in the very short time interval just before the plasma expands to transparency. In the experimental situation, although the total ionization and symmetry are strongly dependent on the actual process by which the solid is transformed into an ionized gas, the process of energy absorption 
in the fraction of the solid that is vaporized. would be expected to be essentially that of the model; viz., uniform absorption. just prior to expansion to transparency. This suggests that not only a prepulse but simply a high power pulse of longer duration could be used to achieve full ionization with high energy. For the prepuise used in these experiments, the increased ionization is obtained, but as the prepulse amplitude increases to the maximum value used, the plasma average energy drops $\sim 30$ percent. However, a drop in average energy, observed also in previous experiments, is not surprising since pre-expansion of the vapor makes it very likely that some of the vapor expands beyond the region in which it can be irradiated by the most intense part of the high power pulse. The reduction or elimination of vapor expansion reaction forces through the use of two-direction irradiation may well make it possible to achieve full ionization without energy loss by using a long duration, high power pulse; however, the data of Figs. 11-13 suggests that, without prepulse vaporization, the plasma would be asymmetric.

The full range of variables on focal properties, laser pulse parameters, and. particle sizes has not yet been explored, and the system is presently being set up with larger particles (75-105 $\mathrm{\mu m}$ diameter) to extend the results to larger quantities of plasma, and later, by variation of laser parameters, to high energies. The results obtained so far, however, confirm the expectations that the new apparatus, using two-direction irradiation of the target provides reproducible, spherically symmetric plasmas of at least $500 \mathrm{eV}$ average energy with particles up to $80 \mu \mathrm{m}$ diameter.

\section{LITE Neutral Beam Line Development}

The energetic neutral beam line for the LITE experiment was designed so that it could be assembled as an independent beam line facility, permitting the development of the neutral beam concurrently with the plasma production and confinement experiments being carried out in the experiment chamber. Parallel development of the neutral beam with plasma production and confinement will permit a developed neutral beam line to be coupled with the experiment chamber approximately midway through the next reporting period. The neutral beam line facility, design was previously discussed (Ref. 1), and a schematic of the design is show in Fig. 16. During this reporting period, significant progress has been made in establishing the neutral beam line facility. The ion source was received and tested at UARL, and fabrication of aII the beam line components (viz., the neutralizer, deflection magnet, drift tube, and beam termination chamber and their respective $\mathrm{IN}_{2}$ dewars) was completed. The component chambers (without the dewars) were polished, leak checked and assembled into a complete neutral beam line as shown in the photograph of Fig. 17. The following subsections will discuss the ion source tests conducted to date and the fabrication and assembly of the entire beam line.

\section{Ion Source Tests}

The ion source is a MATS III source supplied by the Lawrence Livermore Laboratory. Preliminary tests of this ion source on the test stand at the Lawrence Livermore Laboratory were performed with the assistance of UARL personnel (see Ref. 1 ). The data obtained during these tests were presented in Ref. 1 and are also shown in Fig. 18. Initial familiarization tests of the MATS III source to determine its 
operating characteristics using the power supplies available at UARL were conducted with the ion source connected directly to the beam termination tank as shown in Fig. 19. The arc, magnet, and high voltage power supplies were originally obtained under Air Force Contract F-44620-67-C-0082 and subsequently transferred to the ERDA contract. The arc power supply (a NWL $50 \mathrm{Amp}, 500 \mathrm{~V}$, 3-phase, fully rectified, d.c. supply), ballasted with $1.1 \Omega$ in series with the arc, required some modification to prevent the destruction of diodes in the power supply output resulting from high voltage feedback due to ion source fluctuations. The source and shaping magnet power supplies appear adequate for the present operation of the MATS III source. However, the high voltage accelerating power supply is limited to $500 \mathrm{~mA}$, maximum current drain. Since fluctuations in the MATS III source occasionally cause increased current drain, the safe, steady-state operating limit for this power supply is between 350 and $400 \mathrm{~mA}$. A new high voltage d.c. power supply capable of producing 5 Amps at $25 \mathrm{kV}$ has been purchased and is expected early in February, 1975.

In the ion source tests conducted. with the source attached to the beam termination chamber, the extraction grid.s were apertured so that a somewhat larger accelerating voltage could be applied. without exceeding the current limit of the power supply. The aperture covered twenty-six of the original sixty-three $0.46 \mathrm{~cm}$ diameter holes in the MATS III grid, leaving an extraction area $75 \%$ of the original. With this aperturing, tests were conducted up to an accelerating voltage of $10 \mathrm{kV}$. The ion beam was terminated on a 6-inch diameter water-cooled target $170 \mathrm{~cm}$ from the acceleration electrode. Calorimetric measurements of the beam power deposited in the target were made; and the average target current density, corrected for aperturing, is shown in Fig. 18 along with equivalent results obtained at the Lawrence Livermore Laboratory. The data obtained at UARL are in excellent agreement with the LLL results. Parameters of interest in the UARL tests are given in Table $I$.

Table I

$\begin{array}{ll}\mathrm{H}_{2} \text { Flow Rate } & 4.0 \times 10^{-5} \mathrm{gm} / \mathrm{sec} \\ \text { Arc Power } & 4.1 \mathrm{~kW} \\ \text { High Voltage Current (@ 10 kV) } & 385 \mathrm{~mA} \\ \text { Decelerating Voltage } & 1.2 \mathrm{kV} \\ \text { Decelerating Current } & 22 \mathrm{~mA} \\ \text { Pressure* } & 6.0 \times 10^{-5} \text { Torr }\end{array}$

*measured in beam termination chamber

Although the tests at ILL showed that a focusing magnet significantly increased the current density on target (see Fig. 18), in the UARL tests, a beam focusing magnet was not used and this is reflected in the data presented in Fig. 18. A focusing magnet was fabricated. at UARL which provided an axial field of approximately 600 Gauss at the centerline. However, when the magnet was turned on during beam 
operation, excessive current drain occurred at the decel electrode. After consultation with Dr. John Osher of LLL, it was concluded that stray magnetic fields from the focusing magnet were causing an anomalous discharge in the extraction region. Elimination of these stray field.s can be achieved by moving the magnet further away from the extraction electrodes or by employing an iron shunt around the field. coils to provide a return path for the magnetic field lines in close proximity to the coil. Further consideration (and redesign) of the focusing magnet will await additional tests with the complete beam line in operation.

Tests at UARL with accelerating voltages greater than $10 \mathrm{kV}$ were not possible without additional aperturing of the extraction electrode due to the current limit on the existing UARL power supply. It was felt that additional aperturing might distort the data and provide operating conditions that would not be representative when the full electrode extraction area was available. However, as a check on the capability of the experimental setup to withstand higher voltages, the ion source (with no arc and, hence, no beam extraction) was operated to $17.5 \mathrm{kV}$. At this point voltage breakdowns occurred across water lines feeding the high voltage and ground. portions of the ion source. The water lines have been rerouted to eliminate as much as possible the close proximity of lines of different voltages.

Tests to measure the rapidity with which the neutral beam could be turned on were also performed at this time. The length of this "turn on" time is of major importance for the target plasma experiments that will be carried out later because of the possible destabilizing effects of the beam on the suspended LiH particle. While movement of the particle due to beam bombardment is calculated to be small and well within the holding capability of the feedback suspension system, excessive heating and perhaps vaporization of the particle by the neutral beam could occur if the neutral beam. is turned on and incident on the particle for too long a period. before the particle is vaporized by the laser. On the other hand, as discussed in Section III-A of this report, the beam must be turned on within 10 msec after the target plasma has been established if the beam is to overcome the plasma losses. One method that has been employed to rapidly switch on a beam is to switch the arc, leaving the accelerating voltage on at all times (see Ref. 8). This method. was tried with the MATS III source at UARL. Fig. 20 shows the time dependent traces of a) the arc power supply, and b) the ion beam. The arc power supply responds in several milliseconds; however, because of the time required to stabilize the arc, nearly $500 \mathrm{msec}$ was required to establish a steady state ion beam. In the first $300 \mathrm{msec}$ there is no evidence of a beam, while an additional $\sim 150 \mathrm{msec}$ are required for the beam to reach full power. Since the temperature of a $70 \mu$ diameter particle is calculated to rise about $500^{\circ} \mathrm{C}$ in $150 \mathrm{msec}$ for beam currents of approximately $5 \mathrm{~mA} / \mathrm{cm}^{2}$, this method of beam turn-on could be employed in low beam current experiments. Other methods of more rapid beam turn-on are being investigated for use with higher beam intensities. 


\section{Complete Beam Line \\ a. Beam Line Assembly}

As discussed above, the ion source tests were limited by the high voltage power supply restriction. Rather than wait for the new power supply to be delivered. to continue ion source tests, it was decided to assemble the complete beam line and. launch into complete beam line assembly and tests even though the latter may be limited in beam current for a short period of time.

The modular design of the beam line permitted each of the components to be fabricated and inspected separately before being assembled into a complete line. Each of the individual component chambers was fabricated of 300 series stainless steel having a commercial grade number 4 finish. The interior surfaces were closely inspected after being received from the vendor, and any surface imperfections, material voids, dents or scratches and. all welded seams were ground and polished. The close inspection and. surface preparation are necessary to permit the achievement of the high vacuum requirements of the neutral injection experiments. Similar care was taken with the $\mathrm{LN}_{2}$ dewars, also fabricated of stainless steel with a number 4 finish. All flanges $14^{2}$ inches diameter and less are knife edges sealing against a copper gasket, while the larger flanges employ copper "O" ring seals similar to those employed in the experiment chamber. Each of the component beam line chambers were individually leak checked to a leak rate less than $1 \times 10^{-9}$ std. cc helium $/ \mathrm{min}$.

The beam line components were assembled as shown in Fig. 17, and the entire beam line leak checked to the same standard as the individual components. Three 10-inch diffusion pumps and two fore pumps (the neutralizer and drift tube diffusion pump outlets are manifolded together) are employed as shown. Fabrication and assembly of the $L N_{2}$ dewars in the dump tank, drift tube and neutralizer, as well as the water vapor spray heads in the neutralizer have been completed and both the spray heads and. dewars for the neutralizer are presently being installed. (See Fig. 21 for pictorial of the neutralizer.) A photograph of the spray heads installed in the central dewars is shown in Fig. 22. The spray heads previously described in Ref. 1 have 28 in. square glass mosaics, each containing $4 \times 10^{4} 0.1 \mathrm{~mm}$ diameter holes. The glass mosaics, each $2 \mathrm{~mm}$ thick, are arranged. in 7 rows of 4 each (see Fig. 22). The glass has been gold coated ( $\sim 1 \mu$ thick) and grounded to the support structure in order to prevent charge build-up on the glass surface. The neutralizer outriggers and their respective dewars, the final major components of the beam line to be fabricated, have been received and are undergoing final polishing and cleaning. Four additional 10-inch diffusion pumps, one in each of the neutralizer outriggers and one on each side of the drift tube will be added at a later date. The three neutralizer diffusion pump outlets will be manifolded together to be pumped by one 2800 liters/min forepump. A similar arrangement will be used for the three drift tube pumps.

The neutralizer will employ water vapor to charge exchange with the ions to produce a neutral beam. (see Refs. I and 3). An estimated. water vapor flow rate of 
approximately $0.1 \mathrm{gm} / \mathrm{sec}$ will be necessary for neutralization to be effective (Ref. 1). Bench tests were conducted to demonstrate that this amount of water vapor could. be generated. In these tests, a copper cylinder ( 3 in. diameter by 5 in. long) was partially filled with distilled water and the outlet connected to a flowmeter. A throttle valve was located. downstream of the flowmeter, and unheated 3/8 in. O.D. copper tubing connected the throttle valve through a cold trap to a vacuum pump. The distilled water chamber, flowmeter and throttle valve were immersed in a constant temperature water bath maintained between 45 to $50^{\circ} \mathrm{C}$. No liquid water was condensed. in the unheated. line upstream of the cold trap while water vapor flow rates up to $0.12 \mathrm{gm} / \mathrm{sec}$ were measured. with the pressure d.ownstream of the throttle valve remaining about 2 Torr. This system will be employed in initial neutralizer tests, but provisions for remotely monitoring and controlling the water vapor flow will be incorporated. when the neutral beam is coupled to the experiment chamber.

\section{b. Beam Line Tests}

Preliminary ion source tests have been made with the assembled beam line, primarily to test the effectiveness of the deflection magnet in removing the ions from the main neutral beam before they enter the experiment chamber and to insure that the stray fields from the magnet would have no adverse effect on the ion source operation. The deflection magnet pole pieces are $11.4 \mathrm{~cm}$ square, and $9 \mathrm{~cm}$ apart. A $10 \mathrm{~cm}$ diameter Armco iron shunt containing two coils connects the pole pieces. The measured maximum field between the pole pieces was limited to 1600 Gauss by saturation of the iron shunt.

Figure 23 is a photograph of the beam (with no deflecting field applied.) passing through the drift tube and impinging on a water cooled. copper target located at the end of the drift tube. The beam, operating at $9 \mathrm{kV}$, is apertured to $9 \mathrm{~cm}$ diameter prior to entering the deflection magnet. As can be seen, the beam has a small divergence and is also slightly displaced from the center of the target. When a small deflecting magnetic field is applied. (approximately 300 Gauss), the unneutralized. portions of the beam are spread. Although the clarity of the black and. white reproduction is somewhat obscure (Fig. 24), four discreet beams representing the different compositions were observed, viz., I) the undeflected neutrals ( $\mathrm{H}$ and $\mathrm{H}_{2}$ ), 2) the least deflected $\left.\left(\mathrm{H}_{3}{ }^{+}\right), 3\right)$ the intermediate deflected $\left(\mathrm{H}_{2}{ }^{+}\right)$, and 4$)$ the greatest deflected. $\left(\mathrm{H}^{+}\right)$. The relatively large concentration of ions present in the drift tube results from insufficient neutralization. No water vapor spray was employed. in these preliminary tests, but a small amount (approximately $1.0 \times 10^{-3} \mathrm{gm} / \mathrm{sec}$ ) of nitrogen was bled into the neutralizer. Applying a deflecting field of 1500 Gauss resulted. in large deflections of the ions, but had no significant effect on the ion source operation. Additional tests to determine quantitatively the relative ion beam compositions will be made by deflecting each ion beam component to a water cooled. target located well away from the centerline. The effectiveness of the water vapor neutralizer will also be determined by measuring that fraction of the neutralized. beam which can be deflected. 


\section{Future Test Program}

The completed fabrication of all the major beam line components and the receipt of the new high voltage power supply will permit full beam line tests to be conducted early in the next report period. Initially these tests will be made to determine 1) the effectiveness of the water vapor in neutralizing the ion beam, 2) the amount of streaming flux (both water vapor and hydrogen), and 3) the operating limits of the present MATS III source. Because the required density and flow rate of water vapor (i.e., $10^{14} \mathrm{~cm}^{-3}$ and $0.1 \mathrm{gm} / \mathrm{sec}$, respectively) are large, cryopumping on the $\mathrm{LN}_{2}$ dewars of the neutralizer must be relied upon to remove the water vapor from the system. To determine the amount of water vapor streaming flux, a mass analyzer located in the beam termination chamber will measure the partial pressure of the water vapor at that location. Later experiments with the mass analyser mounted to the experiment chamber will permit measurements to be made not only of the water vapor and hydrogen streaming flux, but of the wall reflux as well.

Consultations with personnel at LLL have suggested that substantial increases in beam power might be achievable by pulsing the arc rather than running it steady state. By operating for periods of less than approximately $2 \mathrm{sec}$ with a duty cycle no greater than 10\%, one limit on the MATS III source imposed by excessive heating of the arc anode and zwischen can be circumvented, and arc currents between 50 and 100 Amps using the existing power supplies, should be attainable. Consequently, plans are currently underway to increase the current output of the MATS III source by running the arc in a pulsed. mode, with each pulse having a duration of up to 2 sec. The increased arc current and a corresponding increase in arc gas flow should permit greater neutral beam currents than previously attained at LLL using the MATS III source.

Although the primary function of the neutral beam is to heat and sustain the target plasma, the beam can also be used. as a target plasma diagnostic. Absorption of the beam by the plasma and scattering of the high energy neutrals are two techniques that could. provide valuable information about the plasma density and would complement the microwave data. A further description of these techniques and their implementation are presented in Section II-D.

\section{Diagnostics}

The first experiments in the LITE program will concentrate on the confinement of laser produced target plasma under high vacuum conditions. In order to determine whether or not the confinement is classical, particular emphasis will be placed on measurements of the plasma density and energy as a function of time so that these parameters can be compared. with the improved. Fokker-Planck calculations of these plasma properties.

Results from the earlier experiments have shown that in order to reduce uncertainties in the comparison between the experiment and theory, plasma density measurements should be improved and diagnostics should be added to measure the energy 
of the confined ions and the space potential. of the plasma. Two new diagnostics which will measure the ion energy and plasma potential are being added to the experiment - a fast atom energy analyser and a heavy ion beam probe. These diagnostics are discussed below. Analysis of microwave interferometer measurements (Ref. 3) has indicated that refraction of the microwave beam by the plasma was producing large effects at higher plasma densities so that interpretation of the measurements depended strongly on the geometry of the plasma. In an attempt to reduce the effects of refraction, the antenna system for the interferometer has been improved. These improvements are discussed below in more detail.

With the addition of the neutral beam to the experiment, two additional method.s of measuring the properties of the target plasma become available. By monitoring the transmission of the neutral beam current through the system during the formation and decay of the target plasma, the line density profile of the target can be measured from the decrease in beam transmission due to trapping in the target plasma when the plasma density is above $10^{13} / \mathrm{cc}$ and several percent of the beam is trapped. Over longer time scales, the density of trapped energetic ions in the plasma can be measured. by measuring the charge exchange flux of energetic neutrals. For the condition that $\tau_{\text {target }}<T$ cx HOT is satisfied, the hot ion density should reach a value at time $t$ proportional to . $_{0}^{t} \mathrm{n}+$ (target) d.t. While the large orbits and drifts of the hot ions in the plasma make it difficult to obtain detailed information about the target density, this measurement is a direct measurement of the quality of the laser produced. plasma as a target for neutral injection.

The computer based data handling system discussed in Ref. I has been installed. in the LITE control room and has been employed in acquiring and processing data from the initial experiments. Since all of the required fast interfaces to the computer have yet to be completed, the number of diagnostics that can be handled simultaneously is limited; however, these interfaces are expected to be completed in time for the magnetic confinement experiment. Results of the application of the system to the initial experiments are discussed below. Also discussed below is a more detailed. analysis of streak photographs of the laser produced plasma taken in earlier experiments. From this analysis it appears that this diagnostic could prove useful in determining the time evolution of the geometry of the plasma.

\section{Fast Atom Energy Analyser}

An energy analyser for determining the energy of fast neutral atoms leaving the confined plasma by charge exchange reactions has been constmucted and is being installed in the IITE facility. Figure 25 is a diagram of the energy analyser and. its vacuum system. The analyser is a parallel plate electrostatic analyser producing a $90^{\circ}$ deflection of the re-ionized atoms into four equally spaced particle detectors. The four energy channels cover an energy range of about 1:3. Re-ionization of the fast atoms is produced in a gas cell at the entrance to the analyser. The analyser and gas cell are contained in a vacuum system separate from the main chamber. The 
gas cell is located about $150 \mathrm{~cm}$ from the center of the plasma and the vacuum connection between the two systems is made through a $1 \mathrm{~cm}$ diameter pipe about $50 \mathrm{~cm}$ long in order to reduce the gas conductivity between the two vacuum systems and to provide differential pumping between the gas cell and the main high vacuum system.

Figure 26 is a schematic of the particle detection system. The detectors are channel electron multipliers with $1 \mathrm{~cm}$ diameter apertures. Pulses from the electron multipliers are amplified and fed. into single channel pulse height

analysers. The outputs of the analysers are fed into four 0-15 scalers that actually comprise a 16-bit input buffer to the computer. The count rate from the four energy channels as a function of time during the decay is determined by having the computer periodically store and clear the contents of the input buffer.

\section{Heavy Ion Probe}

A heavy ion probe diagnostic system is being developed to measure the spatially resolved plasma potential and space and time resolved plasma density of both the decaying and the injection heated LITE plasma. In the heavy ion probe a primary beam of energetic charged ions is directed across the plasma where some of the ions experience ionizing collisions with plasma electrons. The resulting doubly charged. energetic ions are separated from the primary beam by the magnetic field, following trajectories which are a function of the field geometry, beam energy, ionization point within the plasma, and plasma potential. For the LITE system, measurements of the secondary beam intensity and energy will be used to determine the plasma density and space potential. Heavy ion probes have been developed for uniform magnetic field devices and the ST Tokamak, a toroidal system. In a cooperative program with UARL, Rensselaer Polytechnic Institute is presently developing the ion beam diagnostic for the LITE facility under ERDA contract AT(11-1)-2229. The minimum B magnetic field. of the LITE magnet has to be treated three-dimensionally and possesses strong spatial gradients. This presents challenges to the technology of beam probing, as the ion orbits are more complicated in the three dimensional field and the gradients cause defocusing of the primary beam with the resultant loss of spatial resolution. Computational methods have been used to select a proper set of ion orbits, and the defocusing effect will be compensated by a linear field. lens to focus the primary ion beam. The diagnostic system will be capable of mapping density and space potential of the confined plasma with spatial resolution of $0.1 \mathrm{~cm}^{3}$, and time resolution $\sim 5-10 \mu \mathrm{sec}(\operatorname{Ref} .4)$.

Figure 27 is a photograph of the fabricated beam probe system attached to the LITE vacuum chamber. The large beam probe chamber with high voltage feed.throughs contains the ion gun and can be isolated. from the LITE vacuum chamber for modification or adjustment by the metal gasket valve provided. The small vacuum chamber contains sweep plates to electronically sweep the primary ion beam, and the ion beam line is attached to the IITE vacuum chamber with a gimbal mount to provide for the necessary adjustment of the beam probe input trajectory. Preliminary alignment of the primary ion beam with the baseball coil magnetic field using scaled values of the beam energy 
and magnetic field strength has been accomplished and final alignment tests are now being run at full magnetic field (Ref. 5). An electrostatic energy analyser, which is the detector for the ion beam probe system, is scheduled for installation in February, and initial measurements will involve density diagnostics following the decay time history of the confined target plasma.

\section{Microwave Interferometer}

The $4 \mathrm{~mm}$ microwave interferometer used in the earlier experiments has been modified for use in the LITE facility. The main modification is a change in the horm-lens system in order to achieve a more tightly focused microwave beam at the plasma. The tightly focused. system has been chosen in order to reduce the effects of refraction on the transmission between the horns. A schematic representation of the system is shown in Fig. 28 . The $7.6 \mathrm{~cm}$ diameter, $15 \mathrm{~cm}$ focal length transmitting antenna produces a focus at the center of the plasma colum about $1 \mathrm{~cm}$ in diameter. Since the wave front of the microwave at the plasma is approximately concentric with the plasma surface and the focal spot size is considerably smaller than the plasma radius, changes in the wave front of the beam by the plasma should be minimal. The receiving antenna has a $5 \mathrm{~cm}$ diameter and a $20 \mathrm{~cm}$ focal length. The larger $f$ number receiver results in a loss of some transmitted power $(\approx 3 \mathrm{db})$ but does not reduce the spatial resolution of the system. This sacrifice in power was made in order to avoid. a conflict between the microwave antenna and the heavy ion probe and will permit their simultaneous use in any experimental shot, thus giving two independent measurements of the density profile.

\section{Data Handling and Reduction}

The LITE computer based data handling system is currently being used to acquire and analyse the data from current probes in the free expansion plasma production experiments. In these experiments the output from the current probes is fed into transient recorders that digitize and store the data at the rate of 10 points per microsecond from each probe. While waiting for the laser to fire, the computer monitors and stores the output from the particle position detectors. When the laser fires, the position of the particle just prior to the laser firing is displayed. on the scope and the data from the transient recorders is transferred to the computer core. The probe data is then saved in a permanent data file on the disk and reduced. for display on the scope. The data reduction involves integrating the current signal to give the total charge collected, and converting the current vs. time information to a charge vs. energy curve and integrating to obtain the average energy. Figure 29 shows the scope display of the data from the current probes. Although the reduction of this data is straightforward, the fact that the reduced data is immediately available aid.s in aligning the laser beam and positioning the particle for optimum plasma production. The plasma parameters to be optimized, total ionization produced, symmetry of current and energy collected by the different probes, and total energy coupled into the plasma, are all displayed immediately after each shot. 
The LITE data handling system is basically designed to handle large throughput rates of data through the extensive use of fast transient recorders. The system's ability to handle large amounts of data and quickly transfer large blocks of data into magnetic disk storage enabled the system to be used in acquiring the data from the particle position detectors during the shakedown phase of operating the particle suspension system. In this application the computer was used to monitor the vidissector outputs on a time scale fast relative to the motion of the particle and yet retain a history of the particle motion over a period of several seconds. It proved to be important to be able to see the behavior of the position detectors over the whole period of time while the feedback system was attempting to gain control and stabilize the particle. This information permitted the tracking of the particle if it was lost by the suspension system and helped in determining the reasons for these premature losses.

\section{Analysis of Plasma Streak Photographs}

Further analyses have been made of the two streak photographs of the mid-plane luminosity of the mirror confined plasmas, which have been reported in Ref. 1 . One of the streak photographs has been reproduced as a figure in that report, and the method of densitometer scanning of the film to measure the transmission of the photographic image is also described there. The transmission data are preserved. as traces on densitometer recorder strip charts. A computer program was written to facilitate the reduction of this data to determine the radial dependence of the optical emission source density at the plasma mid-plane. The scale of the transmission $\mathrm{T}$ is adjusted for a range of 0 (complete opacity) to 100 (complete transmission). The film density $D$ is computed from the transmission as

$$
D=\ln \frac{100}{T} \text {. }
$$

In the absence of exposure calibrations for the films, a relation between film density and exposure $\mathrm{E}$ was assumed, namely

$$
E=10\left(10^{D}-1\right)
$$

so that

$$
D=\log _{10}\left(\frac{E}{10}+1\right)
$$

On the further assumptions that the plasma is axisymmetric at the mid-plane and optically thin, the radial dependence of the optical emission rate is obtained by an Abel inversion of the radial-dependent film exposure. The $20 \times 20$ matrix of transformation coefficients, published in Ref. 9, is used for this computation.

The radial emission distributions calculated. from the densitometer data for the two streak photographs are shown in Figs. 30a and 30b. The two sets of curves differ in both initial power density and in the way they evolve with time. Figure $30 \mathrm{~b}$ correspond.s to the streak photograph discussed in Ref. 1. Since these are the only 
streak photographs that have been made, it is not possible to say which behavior is typical of the LITE mirror confined plasmas. The emission rates of plasma ions are proportional to the product of the electron and ion densities, while the emission rates of excited background neutrals are proportional to the product of the electron and neutral densities. Hence, time-resolved spectral measurements of the optical emission will be required before the plasma density distributions can be inferred. However, the tendency of the curves in Fig. $30 \mathrm{a}$ to flatten for times up to $75 \mu \mathrm{sec}$ is noteworthy, since that is the behavior expected when plasma losses are dominated. by scattering into the loss cones. Since scattering losses are proportional to the square of the plasma density, the radial profile at the plasma mid-plane should. tend. to a uniform density. For elapsed times of $87 \mu \mathrm{sec}$ and larger, the plasma loss rate in the wings of the radial profile appears to be larger than the loss rate at the center. This behavior might be explained by a sudden large increase in the vacuum background density, leading to enhanced charge exchange loss. 


\section{FOKKER-PLANCK AND BUILDUP STMULATION CALCULATIONS}

\section{A. Fokker-Planck Calculations of LITE Plasma Development}

\section{Modifications of the ISOTIONS Code}

ISOTIONS (Ref. 10), a one-dimensional, multispecies Fokker-Planck code developed at the Lawrence Livermore Laboratory for the calculation of particle distribution functions of mirror confined plasmas has been adapted for use on the UARL UNIVAC 1110. This code permits the inclusion of lithium ions in calculations of the time-behavior of the LITE target plasma. During this report period additional modifications have been incorporated in the ISOTIONS code for the IITE calculation. In particular, a) the iteration subroutines have been modified to eliminate negative values of the distribution functions, b) a subroutine which models the plasma losses by charge exchange with the neutral background and the effects of wall reflux of fast neutrals escaping from the plasma has been added, c) new source terms for calculating plasma buildup by injection of energetic neutral beams have been included, and d.) subroutines for the calculation of energy-dependent cross sections and distribution and source averaged rate coefficients have been added. to the code. Each of these modifications is discussed in a subsection below.

\section{a. Iteration Subroutine}

A source of difficulty in the ISOTIONS code was discovered in calculations of electron-ion energy equilibration. Near equilibrium, negative values of the distribution functions were generated and the calculations became unstable. This problem is discussed, in another context, in a paper by Chang and Cooper (Ref. 11) who propose an alternative iteration scheme for numerical solution of Fokker-Planck equations. Their difference equations are of the same form as those used in the ISOTIONS code, but the coefficients are modified in a way that guarantees nonnegative solutions. In subroutine ITERATE of the ISOTIONS code, the calculation of the coefficients $\alpha_{j}, R_{j}$ and $\gamma_{j}$ (cf. Ref. 10) was modified to conform with the Chang-Cooper method. Thus

$$
\begin{aligned}
\alpha_{j}= & \frac{\rho}{x^{2} \Delta x}\left\{\left(1-\eta_{j}\right) A_{j+\frac{1}{2}}^{n+1}+\frac{B_{j}^{n+1}+\frac{1}{2}}{\Delta x}\right\} \\
\beta_{j}= & \frac{1}{\Delta t^{n+1}}-\frac{\rho}{x^{2} \Delta x}\left\{\eta_{j} A_{j+\frac{1}{2}}^{n+1}-\left(1-\eta_{j-1}\right) A_{j-\frac{1}{2}}^{n+1}\right. \\
& \left.-\frac{B_{j}-\frac{1}{2}+B_{j+\frac{1}{2}}^{n+1}}{\Delta x}\right\}+\frac{\rho C_{j}^{n+1}}{x^{2}}
\end{aligned}
$$




$$
\gamma_{j}=\frac{\rho}{x^{2} \Delta x}\left\{\frac{B_{j-\frac{1}{2}}^{n+1}}{\Delta x}-\eta_{j-1} A_{j-\frac{1}{2}}^{n+1}\right\}
$$

in which

$$
\eta_{j}=\frac{B_{j+\frac{1}{2}}^{n+1}}{\Delta \times A_{j+\frac{1}{2}}^{n+1}}-\frac{1}{\exp \left\{\Delta \times A_{j+\frac{1}{2}}^{n+1} / B_{j+\frac{1}{2}}^{n+1}\right\}-1}
$$

With these changes, the equilibration calculations were stabilized and negative values of the distribution functions were eliminated, as promised in Ref. $l l$.

\section{b. Charge Exchange with Background Neutrals}

A new subroutine; VACBAK, for the calculation of the densities of neutrals in the vacuum background and the plasma volume, was added to the ISOTIONS code to permit a self-consistent calculation of charge exchange plasma losses and wall reflux. When charge exchange occurs between a plasma $\mathrm{H}^{+}$ion and a background neutral, the resulting cold ion is ejected into the loss cone while the plasma ion escapes as a neutral across the field lines to join the background neutral population. For the purpose of this calculation, two vacuum regions are distinguished: an interior region between the plasma and the plasma and the baseball coil, and an outer vacuum region between the coil and the vacuum chamber walls. The outer vacuum region is assumed to contain only cold neutrals, i.e., molecular hydrogen at the ambient temperature. Three species of neutral particles are considered in the inner vacuum region: fast $H$ atoms, Franck-Condon neutrals, and cold neutrals. This model is essentially the same as that added to the single species GAIN code, for the same purpose and as it has been discussed in detail in Ref. 3, the discussion will not be repeated here.

After exiting from the subroutine VACBAK with values of the plasma volume neutral densities, a charge exchange loss coefficient is calculated as

$$
H I(v)=v \sigma_{c x}(v)\left(n_{f}+n_{f e}\right)+v \sigma_{H 2}(v) n_{0} .
$$

The cross sections $\sigma_{\mathrm{cx}}$ and $\sigma_{\mathrm{H}_{2}}$ are for charge exchange of plasma $\mathrm{H}^{+}$ions with neutral $\mathrm{H}$ and $\mathrm{H}_{2}$. The energy dependence of $\sigma_{\mathrm{cx}}$ is obtained from Ref. 12, while the value of $\sigma_{\mathrm{H} \text { p }}$ is twice the per-atom cross section given in Ref. 13. Both cross sections are plotted versus energy in Fig. 31. In calculating coefficients for the next time step $\mathrm{Hl}$ is included with the loss cone term. Since the time step is originally chosen to be small relative to the loss time for scattering into the loss cone, a reduction in the time step is made if necessary when charge exchange loss rates become comparable with or larger than the classical scattering loss rates. 


\section{c. Neutral Injection}

The interaction of an energetic neutral beam with the target plasma involves both charge exchange and ionization. Since the target ions have energies of approximately $1 \mathrm{keV}$, charge exchange replaces a $1 \mathrm{keV}$ ion with an ion of about $10 \mathrm{keV}$ energy. Thus charge exchange tends to raise the mean value of the plasma energy, slowing the plasma decay, but does not contribute to the density buildup. only charge exchange with protons in the LiH plasma is considered, although charge exchange with the $\mathrm{Li}^{3+}$ ions may also contribute to plasma buildup. Ionization of the beam atoms occurs in collisions with the plasma ions and electrons. This process adds to the plasma population and, if it balances the classical scattering and charge exchange losses, a steady state is achieved.

The terms which have been added to the plasma Fokker-Planck equation may be written schematically as follows. A charge exchange source corresponding to the addition of an ion from the source distribution

$$
S_{c x}(v)=\frac{I \lambda}{v}(1-\exp (-L / \lambda)) k_{s} f_{s}(v) n_{i} \frac{\left\langle\sigma_{c x} v\right\rangle}{\langle v\rangle_{s}},
$$

a charge exchange loss term, representing the loss of a target ion

$$
L_{c x}(v)=\frac{I \lambda}{v}(1-\exp (-L / \lambda)) k_{i} f_{i}(v) \frac{\left\langle\sigma_{c x} v\right\rangle}{\langle v\rangle_{s}},
$$

and ionization source terms for protons and electrons

$$
\begin{aligned}
& S_{i}(v)=\frac{I \lambda}{v}(1-\exp (-L / \lambda)) k_{s} f_{s}(v)\left\{\frac{n_{i}<\sigma_{i i} v>+n_{e}<\sigma_{i e} v>}{\langle v\rangle_{s}}\right\} \\
& S_{e}(v)=S_{i}(v)
\end{aligned}
$$

Here, I is the neutral particle current in the beam, $I$ is the mean chord length of the beam through the plasma, $\lambda$ is the mean free path of beam neutrals in the plasma, $f_{i}(v)$ and $f_{S}(v)$ are the distribution functions of plasma target ions and of the ions replacing them while $K_{i}$ and $K_{S}$ are the corresponding normalizing factors. The source distribution function $f_{S}(v)$ may be a delta function or a Gaussian function multiplied by a factor proportional to $\mathrm{v}^{-2}$. Although the beam may be collimated, the ions emerging from charge exchange or ionization are assumed to have the same distribution over directions as the plasma loss cone distribution. 
The $\mathrm{v}^{-2}$ weighting factor accounts for the increase in differential volume element in velocity space with increasing $v$. The quantity $\langle v\rangle_{S}$ is the mean speed of the source distribution. The mean free path $\lambda$ is calculated as

$$
\lambda=\left\langle v_{\xi} /\left\{\left\langle\sigma_{c x} v\right\rangle n_{i}+\left\langle\sigma_{i i} v>n_{i}+\left\langle\sigma_{i e} v>n_{e}\right\}\right.\right.\right.
$$

in which $\sigma_{c x}$ is the $\left(\mathrm{H}^{+}, \mathrm{H}\right)$ charge exchange cross section, while $\sigma_{i j}$ and $\sigma_{i e}$ are cross sections for collisional ionization of $\mathrm{H}$ atoms by ions and electrons. Their energy dependences, obtained from Ref. 12, are displayed in Fig. 31.

\section{d. Rate Coefficients}

When the rate coefficients $\langle\sigma v\rangle$ were calculated by integrating the cross section and relative speed of the beam atoms and plasma particles over both velocity distributions, using the subroutines EVALS and GETFG of the ISOTIONS code, the calculation was found to be impractically time-consuming. Alternatively the rate coefficients arecalculated as averages over the distribution of the particle that dominates the relative speed of the reacting pair. Thus, the rate coefficients $\left\langle\sigma_{c x} v\right\rangle$ and $\left\langle\sigma_{i i} v\right\rangle$ are averaged over the source distribution while $\left\langle\sigma_{i e} v\right\rangle$ is an average over the plasma electron distribution.

\section{Fokker-Planck Results}

The values of the LITE plasma and vacuum system parameters, used in calculating the results to be discussed in this section, are summarized in Table II. Unless specified otherwise, the neutral beam was monoenergetic with an energy of $10 \mathrm{keV}$. In most of the calculations completed to date, three plasma species are included, namely, $\mathrm{H}^{+}$ions, electrons and $\mathrm{Li}^{3+}$ ions, and the injected ions are ad.ded to the $\mathrm{H}^{+}$ population. In the four species calculations, the fourth species was the injected ion population added to the plasma by ionization of the neutral beam and charge exchange with the target plasma $\mathrm{H}^{+}$ions. This was done to more conveniently follow the replacement of the target ions by charge exchange.

\section{a. Target Plasma Decay and Injection Buildup in LITE}

For the four species calculations, all three species of the target plasma are assumed to have initial average energies of $500 \mathrm{eV}$. The plasma initial density, $10^{14}$ electrons $/ \mathrm{cm}^{3}$, was chosen so that the bounce frequency of $\mathrm{H}^{+}$ions is higher than the collision frequency; this seems a necessary condition for mirror confinement. The calculated time-dependence of the confined ion and electron densities are shown in Fig. 32 for a $10 \mathrm{ma} / \mathrm{cm}^{2}$ ( $1 \mathrm{Amp}$ distributed over the $100 \mathrm{~cm}^{2} \mathrm{cross}$ section of the LITE target plasma) $10 \mathrm{keV}$ neutral injection beam. The density of injected ions trapped within the plasma is labeled $\mathrm{H}_{\mathrm{B}}{ }^{+}$. During the first 100 $\mu s e c$, the target plasma density decay is not affected by the low density of injected $10 \mathrm{keV}$ ions. The density of $\mathrm{Li}^{3+}$ ions is about 10 percent of the target $\mathrm{H}^{+}$ion density and, since the $\mathrm{Li}^{3+}$ ions are assumed not to interact with the beam, they have a negligible influence on subsequent developments. After $1 \mathrm{msec}$, the depletion 
TABLE II

LITE PARAMETERS

\section{Dimensions}

Plasma

$I=4 \mathrm{~cm}$

$\mathrm{V}=384 \mathrm{~cm}^{3}$

$\mathrm{S}=410 \mathrm{~cm}^{2}$
Interior Vacuum Region

$S_{I}=1.41 \times 10^{3} \mathrm{~cm}_{3}^{2}$

$V_{I}=1.41 \times 10^{4} \mathrm{~cm}^{3}$

\section{Outer Vacuum Region}

$$
\begin{aligned}
& \mathrm{S}_{\mathrm{v}}=1.08 \times 10^{5} \mathrm{~cm}^{2} \\
& \mathrm{~V}_{\mathrm{v}}=2.49 \times 10^{6} \mathrm{~cm}^{3}
\end{aligned}
$$

Surface Area of Interface between Inner and Outer Vacuum Regions: $S_{F}=1410 \mathrm{~cm}^{2}$

Currents: Fast Neutrals $-I=6.26 \times 10^{18} I^{\prime}, I^{\prime}$ in Amperes; Cold Neutrals $-I_{0}=I / 1000$

Sticking Probabilities: Slow $-b_{0}=0.3$; Intermediate $-\dot{b}_{f c}=1.0$; Fast $-b_{f}=0.7$

$$
\text { Slow }-b_{v}=0.3 \text { at vacuum chamber wall }
$$

Knock-Off Probabilities:

Interior Region - Slow: $C_{\text {of }}=0.5, C_{\text {ofc }}=1.0$ (produced by fast and intermed neutrals) Slow: $a_{0}=0$ (produced by plasma ions)

Outer Region - Slow: $a_{1}=0.5$ (produced by plasma ions)

Fraction of Fast Beam Atoms Scattered in the Interior Region: $a_{m}=0$

Initial Densities: Background $-\mathrm{N}_{0}(0)=10^{8} ; \mathrm{N}_{f c}(0)=0 ; \mathrm{N}_{f}(0)=0 ; \mathrm{N}_{\mathrm{v}}(0)=\mathrm{N}_{0}(0)$ 
of the target $\mathrm{H}^{+}$ion density by charge exchange with the neutral beam is noticeable. The target $\mathrm{H}^{+}$and injected $\mathrm{H}_{\mathrm{B}}{ }^{+}$ion densities become equal at $5 \mathrm{msec}$, and, after 30 msec, the plasma ions consist almost entirely of injected $\mathrm{H}_{\mathrm{B}}^{+}$, which build up to $\mathrm{a}$. steady state density of $1.5 \times 10^{12} \mathrm{~cm}^{3}$. The effects of target ion heating by collisional energy transfer from injected ions may be discerned from the energy curves of Fig. 33 at times greater than $3 \mathrm{msec}$. In the steady state, the mean energies of the various plasma species are also coupled to the energy of the $10 \mathrm{keV}$ injected ions through the plasma potential $\varphi$. The positive plasma potential is adjusted to equalize the loss rates of electrons and positive charges. $\mathrm{H}^{+}$ions with energies less than $e \varphi$ cannot be mirror confined and are excluded from the plasma. Similarly, $\mathrm{Li}^{3+}$ ions must have energies larger than 3 e $\varphi$ for mirror confinement. On the other hand, the mean energy of the electrons must be less than e $\varphi$, since electrons with less energy cannot escape from the plasma. The steady state values of the energies of $\mathrm{Li}^{3+}$ and target $\mathrm{H}^{+}$ions are, however, of no practical interest, since their densities are many orders of magnitude smaller than the injected ion density after $0.1 \mathrm{sec}$.

As an altermative to the use of LiH, the LITE target plasma may also be formed by laser irradiation of a lithium deuteride particle. The larger mass of the deuteron should result in a slower decay of the target plasma, and the mass difference may also allow the target ions to be distinguished from injected ions by suitable diagnostics. Results of a calculation of the injection of a beam of $\mathrm{H}$ atoms into a LiD plasma are shown in Fig. 34. On comparison with Fig. 32, it is seen that the $\mathrm{D}^{+}$component of the target plasms is longer-lived than the $\mathrm{H}^{+}$of the $\mathrm{LiH}$ target. As a result, the plasma density does not drop below $10^{12} \mathrm{~cm}^{-3}$ as before, although the subsequent buildup to the steady state density is not remarkably different.

The time-dependent neutral densities in the plasma volume and vacuum regions for the case considered in Fig. 32 are presented in Fig. 35, showing the transient buildup of neutral densities and their decay to a steady state. The initial values of the cold neutral densities, $N_{0}$ and $N_{v}$, were $10^{8} \mathrm{~cm}^{-3}$, while the initial values of fast and Franck-Condon neutrals were zero. The buildup in the density $\mathrm{N}_{\mathrm{V}}$ of the vacuum region outside the baseball coil is caused by loss cone ions from the decaying target plasma. The buildup in the density $\mathrm{N}_{0}$ in the inner vacuum region between the plasma and the baseball coil is due chiefly to those neutrals streaming in from the outer vacuum region. Our main concern is that the density $\mathbb{N}_{0}$ does not grow to values large enough for charge exchange losses to wipe out the target plasma. The pumping capacity of gettered surface areas in the outer vacuum region reduces the transient background neutral densities to their steady state values within $10 \mathrm{msec}$. The mean density of cold neutrals in the plasma volume is $\mathrm{n}_{\mathrm{O}}$ and its ratio to $\mathrm{N}_{\mathrm{O}}$ is the shielding factor. It is apparent from $\mathrm{Fig}$. 35 that for the conditions considered the plasma is well shielded from cold neutrals. The densities of fast neutrals $n_{f}$ and of Franck-Condons $n_{f c}$ in the plasma volume are essentially the same as the corresponding densities $N_{f}$ and $N_{f c}$ in the adjacent vacuum region, 
because their mean free paths in the plasma are of the order of the plasma dimensions and much larger than those of the cold neutrals. Since the charge exchange rate coefficients are approximately the same for all three neutral species, their contributions to plasma charge exchange losses are nearly proportional to their densities in the plasma volume. These results suggest that virtually all of the charge exchange losses are attributable to cold neutrals.

The calculated steady state densities of plasma and neutral background are plotted against neutral beam current in Fig. 36. Between $1 \mathrm{Amp}$ and 16 Amps, the steady state plasma density rises linearly with neutral beam current. The steady state values of $n_{0}$ have a similar dependence on beam current, but $N_{0}$ increases as the product of the current and the plasma density. This is in accord with the expectation that at high density, the shielding factor $\left(\mathrm{n}_{\mathrm{O}} / \mathrm{N}_{0}\right)$ is inversely proportional to plasma density. For the highest beam currents, $\mathbb{N}_{\mathrm{v}}$ is proportional to $\mathrm{N}_{0}$, because in the steady state the strongest source of neutrals in the vacuum region is the streaming of cold neutrals from the inner vacuum region. For beam currents smaller than $1 \mathrm{Amp}$, the plasma is not as effectively shielded from the neutral background, and the charge exchange losses become comparable with the buildup rate of injected ions; for beam currents less than $0.5 \mathrm{Amp}$, no steady state was found.

\section{b. Delayed Injection}

For all of the preceding results, the neutral beam was assumed to be switched on at $t=0$. As discussed in Section II, there may be compelling reasons for delaying the neutral beam for a short time after plasma production. It is clear from examination of the density curves in Fig. 32 that a delay of $100 \mu \mathrm{sec}$ should have no effect on the target plasms decay of the subsequent plasma buildup. On the other hand, a delay as long as $30 \mathrm{msec}$ could result in loss of the target plasma before neutral injection can be effective. Accordingly, in order to determine the effect of a delay on the plasma behavior, two calculations were made in which the beam current was zero for delay times of $1 \mathrm{msec}$ and $10 \mathrm{msec}$. The results of these three species calculations are shown in Fig. 37. The solid curves are the $\mathrm{H}^{+}$, electron and $\mathrm{Li}^{+}$densities for the I msec delay; they differ imperceptibly from the corresponding curves obtained for no delay. The $10 \mathrm{msec}$ delay, although producing the same steady state condition, results in a substantially reduced value of the minimum plasma density. If the minimum value of plasma density during the injection buildup is critical for plasma stability, it would appear that injection delay time should be no larger than $1 \mathrm{msec}$.

\section{c. Distribution Functions}

Initially, the distributions of the ions and electrons are Maxwellians with mean energies of $500 \mathrm{eV}$. The ion distributions rapidly evolve to loss cone distributions, while the electron distribution functions remain nearly Maxwellian because of the plasma potential. Electrons with energies less than e $\varphi$ are trapped and only 
the higher energy electrons can be scattered out of the plasma volume. The $\mathrm{Li}^{3+}$ density is rapidly depleted early in the target plasma decay so that only the $\mathrm{H}^{+}$. ion distribution functions are of concern to us here. As the target plasma decays and the injected $\mathrm{H}^{+}$ion density builds up, the total $\mathrm{H}^{+}$ion distribution evolves very quickly from the loss cone distribution into two distinct groups. It maintains this double-hump shape for tens of milliseconds before relaxing to the final loss cone shape characteristic of the high energy ions. Figure 38 shows the double - hump distribution function calculated at $6.2 \mathrm{msec}$ in the case of monoenergetic beam injected after $1 \mathrm{msec}$ delay. The presence of this double humped velocity distribution can lead to electrostatic instabilities (Ref. 14-16) which have been demonstrated experimentally (Ref. 17) in minimum-B mirror devices.

The double hump instability results from the interaction between the cold and the hot ions. The cold ions act as a dissipative medium for the energy of the hot ions, with the electrostatic instabilities serving as the energy transfer mechanism. The result of the interaction is the rapid cooling of the hot ions. The increased scatter due to this cooling of the hot ions and the presence of the microinstability results in an increased loss of ions through the mirror loss cones. Analysis (Ref. 14) has shown that these instabilities have a lower threshold density than for the drift cone mode (Ref. 18) and hence, cöuld be of concern in any neutral beam heated target experiment.

For the LITE parameters and $\epsilon_{i}=\frac{\omega_{p i}{ }^{2}}{\omega_{c i}} \gg 1$, the double hump modes of interest are those at the higher density described in Ref. 15. High frequency, nonresonant instabilities are predicted for flute type modes if the hot to cold ion density ratio exceeds a threshold,

$$
\frac{n_{H O T}}{n_{\operatorname{COLO}}}>\left(\frac{2 N^{3}}{\pi}\right)\left[\left(N+\frac{1}{2}\right)^{2}-1\right]^{-2}
$$

where $\mathbb{N}$ is the cyclotron harmonic number. The instability occurs at the lower hybrid frequency given by

$$
\omega_{l h}^{2}=\left|\omega_{c i} \omega_{c e}\right|\left\{1+\left|\omega_{c i} \omega_{c e}\right| /\left(\omega_{p i}{ }^{2}+\omega_{c i}{ }^{2}\right)\right\}
$$

with growth rates, $\frac{1}{2} \omega_{c i} N \Delta$, where $\Delta$. is the fractional density of hot ions. If the inequality is reversed, then a sharply resonant instability occurs at multiples of the ion gyrofrequency when the lower hybrid frequency is a multiple of the ion gyrof requency

$$
\omega_{l h}=N \omega_{c i} \quad N=2,3 \ldots
$$

The growth rates for these resonant modes are given by $\omega_{\mathrm{ci}}\left(\frac{\mathrm{N} \Delta}{2 \mathrm{~T}}\right)^{\frac{1}{2}}$. 
From the calculated LITE plasma densities at the times from .I to 10 milliseconds into the plasma decay (see Fig. 32), the instability criterion of equation (14) is satisfied, predicting nonresonant instabilities at the lower hybrid frequency with estimated growth rates initially near $.5 \omega_{c i}$ and decreasing with time to .I $w_{c i}$. Because these lower hybrid modes have such large growth rates, they could severely limit the plasma lifetime if the unstable oscillations can be supported in the plasma. In addition to having large growth rates, the fact that double-hump modes can occur at very low density suggest that the doublehump modes are potentially more dangerous than the drift cone modes which have also been predicted although not yet observed in mirror devices.

This preliminary analysis of the double-hump instability suggests that mirror devices could be subject to such modes and consequently a closer examination of these instabilities - their development, impact on the plasma, decay, and how they would manifest themselves - has been initiated. Moreover, since neutral beam heated target plasmas will always contain both hot and cold ions at some time during the plasma decay, anticipating the impact of the double-hump instability, it may be of value to find means for smoothing the distribution functions.

Rapid modulation of the beam energy suggests itself as ane approach for smoothing the distribution and has been examined in a series of Fokker-Planck calculations. The results of imposing an energy spread of $2 \mathrm{keV}$ at the halfmaximum of the beam distribution function are shown in Fig. 39. The peak-to-valley ratio at $6 \mathrm{msec}$ is somewhat smaller than in Fig. 38, but the distribution is far from smooth. Further calculations shown in Fig. 40 suggest that the neutral beam energy must be modulated by $\pm 50 \%$ of the mean energy in order to reduce the peakto-valley ratio to 2. Rapid modulation of the beam over that large energy range, however, may be impractical. Additional calculations examining the effects of a slow sweep of the energy of one or more monoenergetic beams will be carried out during the next contract period to explore this more tractable approach for smoothing the confined plasma distribution.

\section{d. Initial Conditions of the Mirror Confined Plasma}

As discussed in connection with the initial conditions for the Fokker-Planck calculations, a necessary condition for mirror confinement of a plasma is that the bounce time, or thetime of travel between mirror points, be less than the average time for scattering into the mirror loss cones. When the ion energies are specified, this condition imposes an upper limit on the initial density for mirror confinement calculations. An attempt has been made to model the transition from magnetic field capture, at $\beta=1$, to mirror-confinement.

The cross-field motion of the expanding plasma is stopped at radius $R(0)$ after a few tens of nanoseconds (Ref. 19). Meanwhile, the expansion of the plasma parallel to field may be assumed to continue until the plasma density arops to values at which the bounce time is less than the mean scattering time into the loss cones and the plasma is mirror confined. As the density decreases, so does the plasma 
magnetization and the field in the plasma volume can rise toward its vacuum value. Hence, the plasma radius may become larger than its $\beta=1$ value, $R(0)$, but this feature is not included in the present model. Rather, the plasma is assumed to expand along the axis of the field occupying a volume equal to that of a cylinder of radius $R(0)$ and a length equal to the product of the free expansion boundary speed and the time elapsed after $\beta=I$ trapping. During this time, the Fokker-Planck model allows energy exchange among the ions and electrons, but with the loss cone scattering term set equal to zero. A formula for the time-dependent density of each plasma species can be derived for this one-dimensional expansion by the methods of Ref. 20 as

$$
n(t)=n(0) / h(t)
$$

where

$$
\begin{aligned}
h(t)= & \left\{\left[1+\frac{3}{2} \omega^{2} \uparrow^{2}+\frac{1}{2}\left(\left(4+3 \omega^{2} t^{2}\right)^{2}-4\left(4+3 \omega^{2} t^{2}\right)\right)^{1 / 2}\right]^{1 / 3}\right. \\
& \left.+\left[1+\frac{3}{2} \omega^{2} \uparrow^{2}-\frac{1}{2}\left(\left(4+3 \omega^{2} \uparrow^{2}\right)^{2}-4\left(4+3 \omega^{2} \uparrow^{2}\right)\right)^{1 / 2}\right]^{1 / 3}-1\right\}^{3 / 2}
\end{aligned}
$$

and

$$
\omega=\dot{R}(0) / R(0)
$$

The velocity $R(0)$ is the plasma free boundary expansion speed in the absence of a magnetic field. The plasma densities are scaled in this way, until the bounce time becomes less than the loss cone scatter time, whereupon the density scaling is stopped and the loss cone scatter term is switched on.

The initial conditions on the densities and energies of the ions and electrons are the $B=1$ stopping conditions. Specification of the magnetic field $B$ and the mean energy per plasma particle $\bar{\epsilon}$ is sufficient to determine the densities of the electrons and ions. The hydrogen and lithium densities are equal and in the fully ionized plasma the electron density is four times the hydrogen density $\mathrm{n}_{\mathrm{H}}$ so that, for $\beta=1$,

$$
6 n_{H} \bar{\epsilon}=\frac{B^{2}}{8 \pi}
$$

The initial energies of the ions and electrons are those of the free plasma expansion in which all of the plasma energy is in the form of kinetic energy of expansion (Ref. 20). The expanding plasma is homogeneous and the electrons and two ion species move with the same velocities. Hence their mean energies are proportional to their masses, so that the initial energies are, in terms of $\bar{\varepsilon}$ and the ion masses $m_{i}$ and electron mass, $m_{e}$,

$$
E_{i}=\frac{6 m_{i} \vec{\epsilon}}{m_{e}+\sum_{i} m_{i}}
$$


and for electrons,

$$
E_{e}=\frac{6 m_{e} \bar{\epsilon}}{m_{e}+\sum_{i} m_{i}}
$$

The value of the stopping radius $R(0)$ may be chosen independently of these initial values, but its choice fixes the values of the total number of ions in the laser produced plasma and the LiH solid particle size, as well as the total energy input to the plasma by the laser pulse. The other parameter required for the calculation of $\omega$ is the free boundary expansion speed $\stackrel{R}{R}(0)$. It is related to the energy per unit mass, Ei, in cgs units by (c.f. Ref. 20, Eq. 37)

$$
\dot{R}(0)=\left(10 E_{1}\right)^{1 / 2} \quad .
$$

The calculated time-dependent densities and energies are presented in Figs. 41 and 42 for a target plasma with $\bar{\epsilon}=500 \mathrm{eV}$, and a $\beta=1$ stopping radius of $R(0)=1 \mathrm{~cm}$ in a magnetic field of 10 kilogauss. For the assumed stopping radius, the equivalent solid particle radius is $54 \mu$ and the laser energy input is 2.5 Joules. The axial expansion without mirror losses lasts for about $0.3 \mu \mathrm{sec}$. During this time the electron energy rises from its initial $0.2 \mathrm{eV}$ value to about $50 \mathrm{eV}$, by collisions with the much hotter ions, but the rapid decrease in plasma density limits the energy transfer. After $0.3 \mu \mathrm{sec}$, the plasma is mirror-confined. The electron density at that time is slightly higher than the $10^{14} \mathrm{~cm}^{-3}$ value of the initial. density assumed for the other calculations discussed in this section. But the mean energies of the plasma species are different: $E_{H}=458 \mathrm{eV}, E_{e}=55 \mathrm{eV}$ and $\mathrm{E}_{\mathrm{Li}}=2350 \mathrm{eV}$, instead of $500 \mathrm{eV}$ for all. Since the energy of the lithium ions is now about five times the energy of the hydrogen ions, the latter are scattered out faster than the lithium. Between $10 \mu \mathrm{sec}$ and $300 \mathrm{\mu sec}$, the ratio of densities of lithium to hydrogen is about 5, in contrast to the situation shown in Fig. 36, where this ratio is about 0.I. In spite of this, the hydrogen ion density recovers after about $1 \mathrm{msec}$, as the result of neutral injection. The density rises to the steady state value of $1.5 \times 10^{12} \mathrm{~cm}^{-3}$ obtained for a 1.0 Ampere neutral beam in the calculations reported earlier in this section. Hence, the differences between these results and those discussed in the preceding sections occur during the decay of the target plasma. They arise from differences in assumptions about the rate of energy equilibration among the ions and electrons. It had been assumed, previously, that the equilibration time is very short compared with the transition time from $\beta=1$ to mirror confinement. Diagnostic measurements of the relative abundances of lithium and hydrogen ions in the target plasma will permit a comparison with the density ratios of 0.1 and 5.0 calculated for the two assumptions to determine which is more nearly correct. 


\section{B. Target Buildup Simulation}

\section{Simulation Model}

In the steady state operation of a mirror system sustained by energetic neutral injection, the confined plasma, isolated from material walls by the magnetic field, is enveloped by a background gas which continuously interacts with the plasma by the atomic processes of dissociation, ionization and charge exchange. The charge exchange process represents a net loss of hot ions. since the cold neutral transfers its electron to the hot ion in the plasma, producing a high energy neutral and a low energy ion which is not confined by the magnetic mirror in the presence of the self-consistent space charge potential. Therefore, in order to sustain a dense plasma by energetic neutral injection, it is essential that charge exchange with the background gas be kept to a tolerable level.

It was shown by Simon (Ref. 2l) that for a plasma of sufficiently high line density, the neutral gas flux would be severely attenuated by interactions near the plasma. This phenomena is commonly referred as "burnout". For conditions of controlled fusion interest, however, the mean-free-path. for the cold background neutrals into the hot plasma is very short and all the charge exchange losses occur in a thin layer at the surface. Unless this loss is suitably balanced, the plasma will be eroded away, layer by layer. The sole source of hot ions to balance the losses is ionization of the injected energetic neutrals. Moreover, because the ions are tied down by the confinement magnetic field lines to motion bounded by drift surfaces, only those which are ionized within a layer on the order of a gyro-diameter of the surface can be effective in replenishing the charge exchange losses at the surface. A rough criteria for sustenance, therefore, is that the rate of ionization within a gyro-diameter at the surface exceed the incident flux of the background neutrals times the ratio of the charge exchange crossection to the total crossection for their interaction with the plasma (Ref. 22).

The rate of ionization, however, is dependent on the plasma density at the surface where the scale length for the density gradient is on the order of the iongyro-diameter. Therefore, in order to quantatively determine the condition for sustenance, it is necessary to consider variation over distances comparable to a gyro-diameter as well as the orbital motion of the hot ions.

In a magnetic confinement system, the orbital or gyration time is much less than the time at which the particle distribution evolves due to collisions. Therefore, on the time scale of interest (the collision time), the position of each particle will be smeared out and must be represented by a probability function, which can be calculated from the particle equation of motion about some reference point. In a uniform magnetic field, the obvious choice for the reference point is the position of the guiding center. More generally, in a multi-dimensionally inhomogeneous magnetic field, it is possible to introduce a drift center about 
which the guiding center also oscillates. The time evolution of the distribution of drift centers, which contains the information of interest can then be followed by consideration of the interaction of the probability functions. Conceptually, this may be visualized as the representation of a point particle in three dimensional space by a cloud image formed by time lapse photography in an abstract one dimensional space formed by axial projection to a plane and azimuthal folding to a radial line. Formally, this approach is equivalent to the expansion of the guiding center distribution into a power series in the small parameter $\nu / \omega$, the ratio of the collision frequency to the frequency of orbital motion, and solving for the perturbation of the distribution function resulting from collisions by considering the known probability functions associated with the zero order distributions.

The above discussion serves as the basis for the construction of a computational model to simulate the decay and neutral injection buildup of a magnetically confined plasma in the presence of a background gas. As shown in Fig. 43 a uniform confinement magnetic field is applied in the $z$-direction of a Cartesian coordinate system. The plasma is assumed to be a slab with inhomogeneities only in the $x$ direction and is bounded by two plane walls parallel to the $y-z$ plane. Initially, the plasma slab is made up of space charge neutralized target ions only and is uniformly irradiated from both sides along the $x$-axis by a beam of energetic neutrals as well as a flux of molecular hydrogren at ambient temperature. As the energetic neutrals are trapped by charge exchange and by ionization, a hot plasma population is built up with an energy given by the energy of the injected neutrals. Therefore, the plasma density is made up of two groups of ions, one at the target temperature and one at the beam energy. According to the previous discussion, it is necessary to distinguish between the distribution of density of drift centers which is used for accounting purposesand the distribution of actual density by which the physical interactions occur. The density distribution $n_{j}(x, t)$ is related to the guiding center distribution $n_{j g}(x, t)$ by the expression

$$
n_{j}(x, t)=\int_{-r_{i}}^{r_{i}} n_{j g}\left(x^{\prime}, t\right) w\left(x-x^{\prime}\right) d x^{\prime}
$$

where the subscript $j$ denotes ions of the $j$ th group ( $j=t$ for target plasma and $p$ for the beam created hot plasma) and $W_{j}\left(x-x^{\prime}\right)$ is a weighting function which expresses the probability that a particle whose drift center is at $x^{\prime}$ will be found at $x$. The integral is evaluated in the range within which the excursion of a particle about its drift center can reach $x$. In the case of a homogeneous magnetic field, the particles are tied to the field lines and the drift center is given by the guiding center with the range limited to a gyro-radius about the guiding center.

Since the plasma is immersed in a background of molecular hydrogen gas, it is uniformly irradiated by a gas flux $F_{g}\left(x_{W}, t\right)$ whose intensity is specified by wall conditions at $x_{\mathrm{W}}$ and is attenuated by the plasma. The spatial attenuation of the gas flux is given by 


$$
\Delta F_{g}(x, t)=F_{g}(x, t) \exp \left\{-\int_{x}\left[n_{t}\left(x^{\prime}, t\right) \sigma_{g t}+n_{p}\left(x^{\prime}, t\right) \sigma_{g p}\right] d x^{\prime}\right\}
$$

where $\sigma_{\alpha \beta}$ denotes the effective cross section for the destruction of a projectile of the $\alpha$-species in a gas of $\beta$-species (in the case where the velocity of the projectile is much less than the average velocity of the particles in the host gas, the effective cross section is the actual crossection at the relative energy of interaction enhanced by the ratio of the velocity of the host particle to that of the projectile.

The molecular hydrogen incident on the plasma is assumed to be destroyed by charge exchange and by ionization, resulting in the creation of an $\mathrm{H}_{2}{ }^{+}$which dissociates into a hydrogen ion and a Franck-Condon neutral, each with an energy of about $10 \mathrm{eV}$. Because of the very low energy, the hydrogen ion is accelerated out of the confinement region by the space charge potential and is not contained. The Franck-Condon neutral, on the other hand, will further interact with the plasma by the charge exchange and ionization processes. Since there is a correspondence between the destruction of the molecular hydrogen and the creation of a Franck-Condon neutral, the source function for the Franck-Condon neutrals is given by the attenuation furiction for the molecular hydrogen gas

$$
\int_{x} S_{f} d x^{\prime}=\Delta F_{g}
$$

where the subscript $f$ denotes the Franck-Condon neutrals.

This flux of Franck-Condon neutrals is attenuated by interaction with the plasma according to

$$
\Delta F_{f}=\int_{x} \frac{1}{2} d x^{\prime} S_{f}\left(x^{\prime}, t\right) \exp \left\{-\int_{x^{\prime}}\left[n_{f}\left(x^{\prime \prime}, t\right) \sigma_{f t}+n_{p}\left(x^{\prime \prime}, t\right) \sigma_{f p}\right] d x^{\prime \prime}\right\}
$$

where the factor $\frac{1}{2}$ in the source function arises from the assumption that the product of the dissociation process is directed in either of the two directions with equal probability. The plasma is also irradiated by a beam of energetic neutrals whose intensity $\mathrm{J}\left(\mathrm{x}_{\mathrm{W}}, \mathrm{t}\right)$ can be specified at the wall and is attenuated by the plasma according to

$$
\Delta J(x, t)=J(x, t) \exp \left\{-\int_{x}\left[n_{t}\left(x^{\prime}, t\right) \sigma_{b t}+n_{p}\left(x^{\prime}, t\right) \sigma_{b p}\right] d x^{\prime}\right\}
$$

where the subscript $b$ denotes projectiles of the energetic neutral beam. 
In addition to interaction with the background neutral and the energetic beam, the charged particles also interact among themselves by Coulomb forces, leading to rapid randomization of the velocity vector, and on a slower time scale, equi-partition of energy. In a mirror system, however, scattering of the velocity vector leads to the escape of the particle through the mirror loss cones and therefore, the dominant effect of the Coulomb interaction is a loss process characterized by a mirror loss frequency $\nu_{\alpha \beta}\left(\mathrm{E}_{\alpha \beta}, n_{\beta}(x, t)\right)$ for loss of ions of $\alpha$-species in a plasma of $\beta$-species. The frequency is a function of the relative energy of interaction, $\mathrm{F}_{\alpha \beta}$ and the density of the host particle. Scattering of the velocity vector also leads to particle transport by diffusion. However, because of the mirror loss, this process is insignificant and has been neglected. The relaxation in energy, although it occurs on a slower time scale, may be important in determining the microstability of the system. The generalization of a two group model to a detailed energy distribution and incorporation of energy relaxation by the use of a suitable collision model is straight forward, although computationally complex, Since the microstability of the system is not the subject of concern here, the energy relaxation has also been neglected.

Equations (25-28) together with the mirror loss frequencies account for the physical processes incorporated into the computational model which describes the time evolution of the charged particle density distribution. For the target plasma, the density distribution satisfies the equation

$$
\begin{aligned}
\frac{d}{d \dagger} \int_{x} n_{f}\left(x^{\prime}, t\right) d x^{\prime}= & -\left\{\left[\left(\frac{\sigma_{c x g \dagger}}{\sigma_{g t}}\right) \Delta F g(x, t)+\left(\frac{\sigma_{g x f t}}{\sigma_{f \dagger}}\right) \Delta F_{f}(x, t)\right] \frac{n_{f} v_{f}}{\left(n_{f} v_{f}+n_{p} v_{p}\right)}\right. \\
& +\frac{\sigma_{c x b t}}{\sigma_{b \dagger}} \frac{n_{f}}{\left(n_{\dagger}+n_{p}\right)} \Delta J(x, \dagger) \\
& \left.+\int_{x} n_{f}\left(x^{\prime}, t\right)\left[\nu_{f \dagger}\left(x^{\prime} ; t\right)+\nu_{t p}\left(x^{\prime}, t\right)\right] d x^{\prime}\right\}
\end{aligned}
$$

where $\sigma_{\operatorname{cx} \alpha \beta}$ is the charge exchange cross section for projectiles of species $\alpha$ in a density of species $\beta$ and the cross section ratio accounts for the fact that in the attenuation of the neutrals, only the charge exchange process leads to direct loss of the charged particles. The ratios $n_{t} v_{t} /\left(n_{t} v_{t}+n_{p} v_{p}\right)$ in the first term and $n_{t} /\left(n_{t}+n_{p}\right)$ in the second term of $E q .(29)$ account for the fraction of neutrals destroyed by charge exchange due to interactions with the target ions. For the beam created hot plasma, the guiding center density is built up locally by the trapping of the energetic neutrals and is given directly by. $\Delta J(x, t)$ from Eq. (28; as 


$$
\frac{d}{d t} \int n_{p g}\left(x^{\prime} t\right) d x^{\prime}=\Delta J(x, t)
$$

The equation for the loss of the hot plasma density is completely analogous to Eq. (29) and is given by

$$
\begin{aligned}
\frac{d}{d t} \int_{x} n_{p}\left(x^{\prime}, t\right) d x^{\prime}= & -\left\{\left[\frac{\sigma_{c x g p}}{\sigma_{g p}} \Delta F_{g}(x, t)+\frac{\sigma_{C x f p}}{\sigma_{f p}} \Delta F_{f}(x, t)\right] \frac{n_{p}}{\left(n_{p}+n_{f}\right)}\right. \\
& +\frac{\sigma_{C x b t}}{\sigma_{b t}} \frac{n_{p}}{\left(n_{p}+n_{f}\right)} \Delta J(x, t) \\
& \left.+\int_{x} n_{p}(x, t)\left[u_{p t}\left(x^{\prime}, t\right)+u_{p p}\left(x^{\prime}, t\right)\right] d x^{\prime}\right\}
\end{aligned}
$$

It should be noted that the trapping of energetic neutrals by charge exchange and ionization results in the creation of a hot ion guiding center at the coordinate position where the interaction occurred. In the charge exchange process however, a hot ion is also lost and this loss process is accounted for by the second term in Eq. (30). This gain and loss is treated separately because the guiding center of the particle that was neutralized could lie in a range within a gyro-radius to either side of the site of interaction and there is a diffusive particle transport associated with such charge exchanges which could be important. The mechanism for this transport process can be understood by referring to Fig. 44. Consider an energetic neutral that undergoes a charge exchange interaction with a plasma ion at $x_{0}$ and becomes trapped as a charged particle with its guiding center at $x_{0}$. The ion which became neutralized in the charge exchange process must have been located at $x_{0}$ at the time of interaction, but its guiding center must have lain on a circle of radius $r_{i}$ equal to its gyro-radius about the point of interaction $x_{0}$. Since the ion is now neutralized, it is no longer confined and its guiding center is lost to the system. There-. fore, the charge exchange process, occurring at $x_{0}$, tends to concentrate guiding centers which are originally distributed in the range $x_{0}-r_{i}$ to $x_{0}+r_{i}$ about $x_{0}$. If the density of guiding centers is inhomogeneous, then the ion which participated in the interaction is more likely to have had its guiding center on the high density side. On balance, then, there is a net transport of guiding centers from the high density region to the low density region, and the rate of transport is proportional to the density gradient and the frequency of charge exchange interaction. For atomic hydrogen, the charge exchange process is the dominant interaction at energy below $40 \mathrm{keV}$; in this energy range, therefore this transport process is important in an inhomogeneous system. 
The computational model for the time evolution of the guiding center distribution is now complete. To carry out the simulation, one chooses a spatial interval $\Delta x$ that is small compared to the ion gyro-radius $r_{i}$ and a time step $\Delta \tau$ which is in the range $1 / \omega \ll \Delta T \ll 1 / \nu$ where $\omega$ in this case, is the ion gyro-frequency. Using the weighting function, the density distribution is computed from the initial guiding center distributions. To proceed in time, the recurring computational steps are:

(1) Calculate the local change of plasma density due to the loss processes,

(2) Attribute the density change to changes in guiding center distribution,

(3) Calculate the buildup of guiding centers due to trapping of the beam neutrals,

(4) Update the zero order guiding center distribution,

(5) Recompute the density distribution from the guiding center distribution and march onto the next time step by repeating the computational procedure.

\section{Results of Simulation}

Using the computational code described above, calculations have been carried out to examine the effect of the background gas and the energetic neutral beam on a mirror confined target plasma. For the sample calculations, we consider the infinite plasma slab bounded by two absorbing plane parallel walls of Fig. 43. The plasma is irradiated from both sides by incident background molecular hydrogen gas and energetic atomic hydrogen beam so that the problem is symmetric about the midplane. The computation and the results are displayed for the half-plane only. It should be noted that in a real system, which is more or less cylindrical, the guiding centers will execute azimuthal drift associated with the inhomogeneous magnetic field. However, this drift time is much less than the collision times and therefore, on the time scale of the computation, there is rapid azimuthal mixing of the plasma (but not radial) and therefore, the infinite slab model can be a satisfactory representation of the cylindrical plasma.

For the series of calculations, we consider a system whose full width is $11.6 \mathrm{~cm}$ and is filled with a $500 \mathrm{eV}$ target plasma localized within the center half of the system. As a point of reference, the full width of the system corresponds to 8 gyroradii for $10 \mathrm{keV} \mathrm{H}{ }^{+}$ions in a $10 \mathrm{kilogauss}$ magnetic field, parameters which are typical of the present generation of mirror experiments. Some of the basic physical processes that are contained in the calculations and their effects are shown in Fig. 45. In Fig. 45a, the $500 \mathrm{eV}$ target plasma which filled half the system at an initial density of $10^{14} \mathrm{~cm}^{-3}$ is allowed to decay in a perfect vacuum. The curves shown are the density as a function of position computed from the guiding center distribution with time as a parameter. Because of the finite gyro-radius effect, the density is finite in a region one gyro-radius away from the edge of the guiding center distribution. As noted before, radial transport across the magnetic field is negligible in comparison to axial losses due to Coulomb scattering into the mirror loss cones and the former process is neglected in the code. Therefore, in the Coulomb 
decay of the target plasma shown in Fig. 45a, there is no spatial diffusion. At the edge of the plasma, the density distribution develops a ripple in time. This. can be simply explained. Although the target plasma guiding center distribution is assumed initially, to be a step function, because of the finite gyro-radius, the density gradient is developed over a gyro-radius. Since loss rates are determined by the local density, the particles whose guiding centers are on the edge suffer fewer collisions than those in the interior because they spend a significant fraction of their time in the low density boundary layer. Consequently, as the plasma undergoes Coulomb decay in time, the guiding center distribution develops a negative gradient towards the interior of the plasma. When the density distribution is computed from the guiding center distribution by weighting according to Eq. (24), the effect is smoothed out and the ripple results. The amplitude of the ripple can be reduced by the use of finer spatial grids; however, this effect is not particularly significant as long as it is recognized and understood.

In Fig. 45b the same target plasma is allowed to decay in a background gas of molecular hydrogen which envelopes the plasma with an incident flux intensity of 1016 molecules per $\mathrm{cm}^{2} \mathrm{sec}$, corresponding to a background pressure of about $3 \times 10^{-6}$ torr. The molecular hydrogen dissociates into Franck-Condon neutrals which further interact with the plasma according to the model described previously. As illustrated by the curve in Fig. $45 \mathrm{~b}$ at $\mathrm{t}=.152 \times 10^{-3} \mathrm{sec}$, the Coulomb decay of the target plasma is virtually unaffected. by the presence of the background gas on a millisecond. time scale, but on longer time scales erosion of the plasma surface begins to appear. While the plasma is opaque to the molecular hydrogen gas at room temperature, it is relatively thin to the more energetic Franck-Condon neutrals. As a result, the decay of the plasma density is enhanced throughout the entire plasma volume in comparison to the case shown in Fig. $45 \mathrm{a}$ where the plasma decay occurs in perfect vacuum. It is worth noting that in most actual experiments where the density decay is of interest, the actual quantity that is measured is usually the line density $\int \mathrm{ndx}$ and the interpretation of a density decay is not unique. Calculations such as those displayed in Fig. 45 are of value because they can be used either to predict the line density signal for comparison with the measurements or to relate the measured line density to the actual density profile.

In Fig. $45 \mathrm{C}$ is calculated the injection of $10 \mathrm{keV}$ atomic hydrogen neutrals onto the $500 \mathrm{eV}$ target plasma in the same molecular hydrogen gas background as the case shown in Fig. 45b. The density profiles of the target plasma which has an initial density of $10^{13} \mathrm{~cm}^{-3}$ are shown as dashed curves while the density profiles of the hot plasma created by trapping of the energetic neutral beam are shown as the solid curves. The intensity of the injected energetic neutral beam is $10^{17}$ particles per $\mathrm{cm}^{2}$ per second which corresponds to an equivalent beam current of $16 \mathrm{~mA} / \mathrm{cm}^{2}$. It is seen that the buildup of the energetic plasma due to trapping of the injected beam on the target plasma is very rapid and in this case, attains a maximum value at $.154 \times 10^{-3} \mathrm{sec}$. Because the gyro-radius of the energetic particles is much greater than that of the target plasma, the density of the energetic plasma reaches out beyond that of the target plasma. As a result, it is exposed to the unattenuated 
background gas flux and is preferentially eroded in comparison to the target plasma. Consequently, in this case the effect of energetic neutral injection is only transitory and eventually, both the target plasma and the beam created plasma are eroded away by the background neutrals.

In order to examine sustenance, the beam intensity was increased by an order of magnitude and another series of calculations were carried out. Using the same target as before and in perfect vacuum, the result of injection is shown in Fig. 46 . The density profile for various times is shown in Fig. 46a and in this case, an equilibrium profile is established in about $0.15 \mathrm{sec}$. In this case since there is no background gas the beam created plasma is seen to fill up the entire volume and vanishes at the wall where the boundary condition is that any guiding center whose orbit intercepts the wall is absorbed. The buildup of the hot plasma as a function of time at the center of the plasma is shown in Fig. 46b. The initial rise to an intermediate level and subsequent equilibrium is characteristic of this type of nonlinear system and its shape is primarily a function of the beam intensity and energy.

To examine the effect of a background gas on the beam sustained plasma, a molecular hydrogen gas flux of intensity $10^{12} \# / \mathrm{cm}^{2}-\mathrm{sec}$ was added onto the system and the results, shown in Fig. 47 are seen to be virtually identical to the perfect vacuum case of Fig. 46. To continue the investigation, the background gas flux intensity was increased to $1015 \mathrm{H} / \mathrm{cm}^{2}-\mathrm{sec}$, corresponding to a background pressure of $3 \times 10^{-7}$ torr and the results are shown in Fig. 48. In this case, as can be seen from the density profile in Fig. $48 \mathrm{a}$ the initial buildup was vertually identical to the high. vacuum case, however, referring to Fig. 48b, the buildup attains only the intermediate level before erosion by the background gas becomes dominant and as a result, no sustenance was achieved. As noted before, because the plasma is relatively transparent to the more energetic Franck-Condon neutrals, erosion occurs more or less as a volume process rather than a surface process.

In conclusion, the computation code discribed above can be used to simulate mirror confined plasma decay and neutral injection sustenance experiments. It is useful for the prediction and interpretation of experimental results and for design studies of future systems. A number of refinements to the code are readily feasible. By suitable modification of the weighting function defined by Eq. (24) the code can treat spatially inhomogeneous two or three dimensional problems without the need of actually calculating at each grid point on a multi-dimensional grid. The extension of the two group distribution function to a multi-group distribution is also straight forward and by the incorporation of a collision operator, the energy relaxation of the injected particles in an inhomogeneous medium can be studied in detail. Thus, the essential features of injection experiments can be incorporated into the computation and the code therefore serves as a highly convenient and controlled means for investigating a variety of confinement experiments. 


\section{REFERENVES}

1. Haught, A. F., D. H. Polk, J. T. Woo, W. J. Fader, R. G. Tomlinson, R. A. Jong, W. B. Ard, A. E. Mensing: High Beta Capture and Mirror Confinement of Laser Produced. Plasmas, Semiannual Report, July, 1974, UARL N951514-21, C00-2277-4, prepared under Contract AT(11-1)-2277 for the U. S. Atomic Energy Commission.

2. Haught, A. F., D. H. Polk, J. T. Woo, W. J. Fader, R. G. Tomlinson, R. A. Jong, W. B. Ard: High Beta Capture and Mirror Confinement of Laser Produced. Plasmas, Semiannual Report, July, 1973, UARL M921514-19, C00-2277-2, prepared. under Contract $A T(11-1)-2277$ for the U. S. Atomic Energy Commission.

3. Haught, A. F., D. H. Polk, J. T. Woo, W. J. Fader, R. G. Tomlinson, R. A. Jong, W. B. Ard: High Beta Capture and Mirror Confinement of Laser Produced Plasmas, Semiannual Report, January, 1974, UARU N921514-20, C00-2277-3, prepared under Contract $A T(I I-1)-2277$ for the U. S. Atomic Energy Comission.

4. Stufflebeam, J. H., "Ion Beam Probe System for the UARL Baseball Device", RPDL Report No. T3-7, November 1973.

5. Stufflebeam, J. H., W. C. Jennings, and R. L. Hickok, "An Ion Beam Probe Diagnostic System for the LITE Experiment", RPDI Report No. 74-7, November, 1974.

6. Haught, A. F. and. D. H. Polk, Phys. Fluid.s, 13, 2825 (1970).

7. Goldman, E. B., W. Leising, A. Brauer, and M. J. Lubin, J. Applied Phys., 45, $1158(1974)$.

8. Davis, R. C., et al., Oak Ridge National Laboratory Report ORNL TM-4657.

9. Bockasten, K., J. Optical Soc. of Americ, 21, 943 (1961).

10. Mirin, A. A., Lawrence Livermore Laboratory Report UCRL-51616 (1974).

11. Chang, J. S., and. G. Cooper, J. Computational Physics, 6, I (1970).

12. Riviere, A. C., Nuclear Fusion, 11, 363 (1971).

13. Tawara, H., and. A. Russek, Rev. Mod. Phys., 45, 178 (1973).

14. Hall, L. S.,W. Heckrotte and T. Kammash, Phys. Rev., 139, Al117 (1965).

15. Pearlstein, L. D., M. N. Rosenbluth, and. D. B. Chang, Phys. F1., 2, 953 (1966).

16. Fowler, T. K., Nuclear Fusion, 2, 3 (1969). 


\section{REFEREINCES Cont.}

17. Perkins, W. A., and W. L. Barr, Phys. FI., 11, 388 (1968).

18. Callen, J. D., Center for Space Research Report, No. CSR T-68-3, Massachusetts Institute of Technology, 1968.

19. Haught, A. F., D. H. Polk, and W. J. Fader, Phys. of Fluids, 13, 2842 (1970).

20. Fader, W. J., Phys. Fluid.s, 11, 2200 (1968).

21. Simon, A., Phys. Fluids, I, 495 (1958).

22. Fowler, T. K., "Charge Exchange at the Surface of Mirror Confined Plasmas", to be published in Plasma Physics. 
FIGURE 1. Heavy Ion Beam Pulse Diagnostic System

FIGURE 2. Experiment Chamber Facility - Southwest View

FIGURE 3. Experiment Chamber Facility - Northwest View

FIGURE 4. Experiment Chamber Facility - Northeast View

FIGURE 5. Experiment Chamber Interior - Viewed From Above

FIGURE 6. Experiment Chamber Interior - Viewed From Below

FIGURE 7. Position Signals During Capture of Orbiting Particle

FIGURE 8. Oscilloscope Display of Particle Position

FIGURE 9. Feedback Controlled Particle Position

FIGURE 10. . Vidissector Signals

FIGURE 11. Charge Collector Current - One Direction Irradiation

FIGURE 12. Charge Collector Current - One Direction Irradiation

FIGURE 13.

FIGURE 14.

Charge Collector Current - Two Direction Irradiation - No Prepulse

FIGURE 15.

Charge Collector Current - Two Direction Irradiation - Maximum Prepulse

Effect of Prepulse on Plasma Energy

FIGURE 16. Elevation View of Lite Energetic Neutral Beam Line For Beam Evaluation Without Experiment Chamber

FIGURE 17. Lite Neutral Beam Line Without Experiment Chamber

FIGURE 18. Measured Performance of Mats-III Ion Source - UARL Copy

FIGURE 19. Elevation View of Ion Source Coupled to Beam Termination Chamber

FIGURE 20. Time Dependent Response of Arc Power Supply and Ion Beam

FIGURE 21. Illustration of Cross-Beam Neutralizer

FIGURE 22. Assembled Neutralizer Spray Heads and Dewars

FIGURE 23. Undeflected Beam in Drift Tube

FIGURE 24. Beam Deflected By Magnet

FIGURE 25. Schematic of Fast Atom Energy Analyser

FIGURE 26. . Block Diagram of Fast Atom Energy Analyser Electronics

FIGURE 27. Heavy Ion Beam Pulse Diagnostic System

FIGURE 28. Microwave Interferometer

FIGURE 29. Computer Display of Data from Current Collectors

FIGURE 30. Radial Dependence of the Optical Emission at the Plasma Midplane

FIGURE 31. Ionization and Charge Exchange Cross Sections

FIGURE 32. Lite Plasma Densities

FIGURE 33. Lite Plasma Energies

FIGURE 34. Lite Plasma Densities-LiD

FIGURE 35. Lite Neutral Densities

FIGURE 36. Lite Steady State Densities vs Current

FIGURE 37. Lite Plasma Densities

FIGURE 38. Proton Distribution Functions

FIGURE 39. Proton Distribution Functions

FIGURE 40. Proton Distribution Functions 


\section{LIST OF FIGURES (Cont'd)}

FIGURE 41. Lite Plasma Densities

FIGURE 42: Lite Plasma Energies

FIGURE 43. Computational Model for Neutral Injection into Target Plasma

FIGURE 44. Mechanism for Charge Exchange Diffusion Process

FIGURE 45. Simulation of Basic Processes in Plasma Decay and Injection Sustenance

FIGURE 46. Sustenance in Perfect Vacuum

FIGURE 47. Sustenance in High Vacuum

FIGURE 48. Injection into Target Plasma in Poor Vacuum 


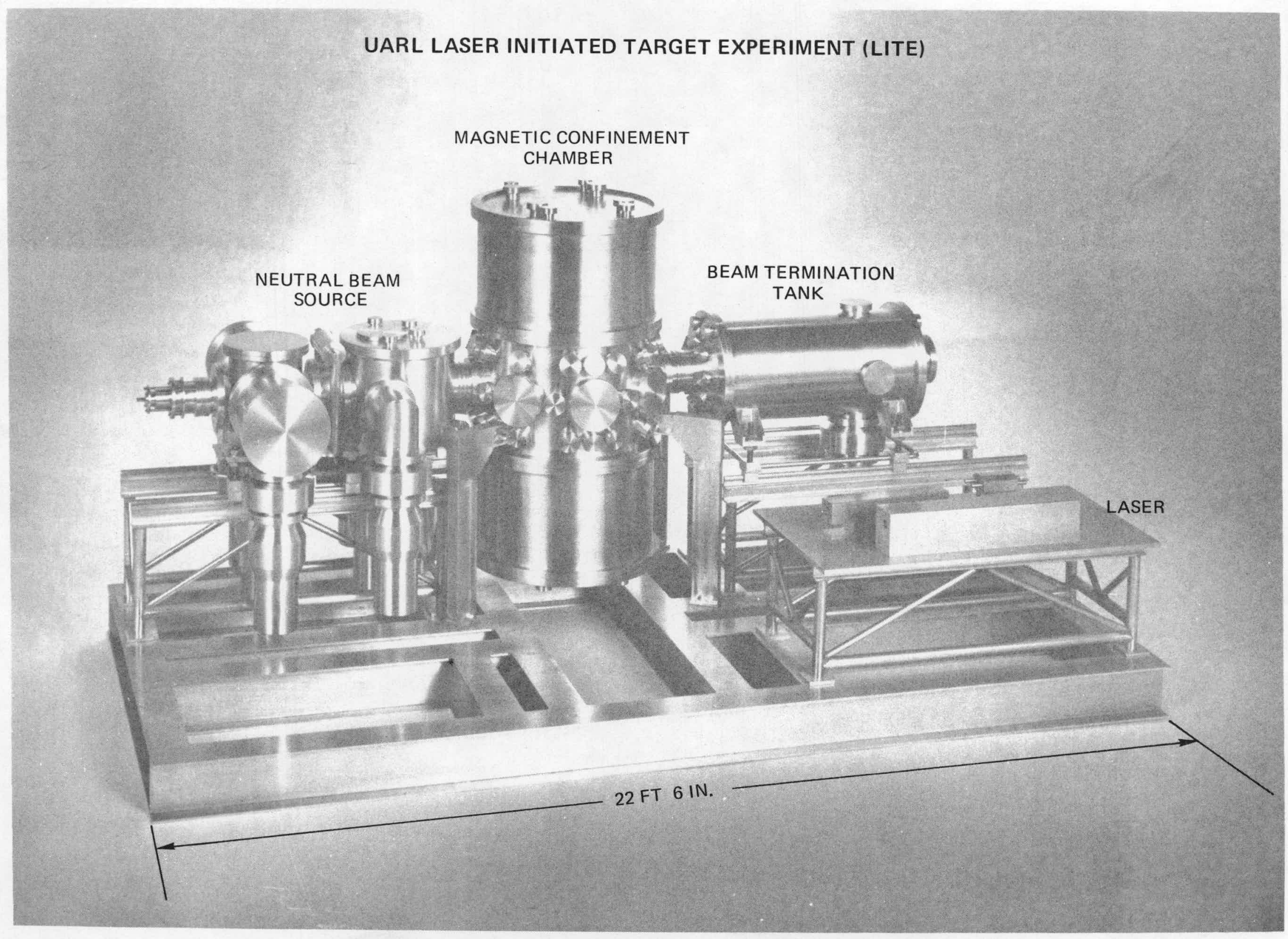




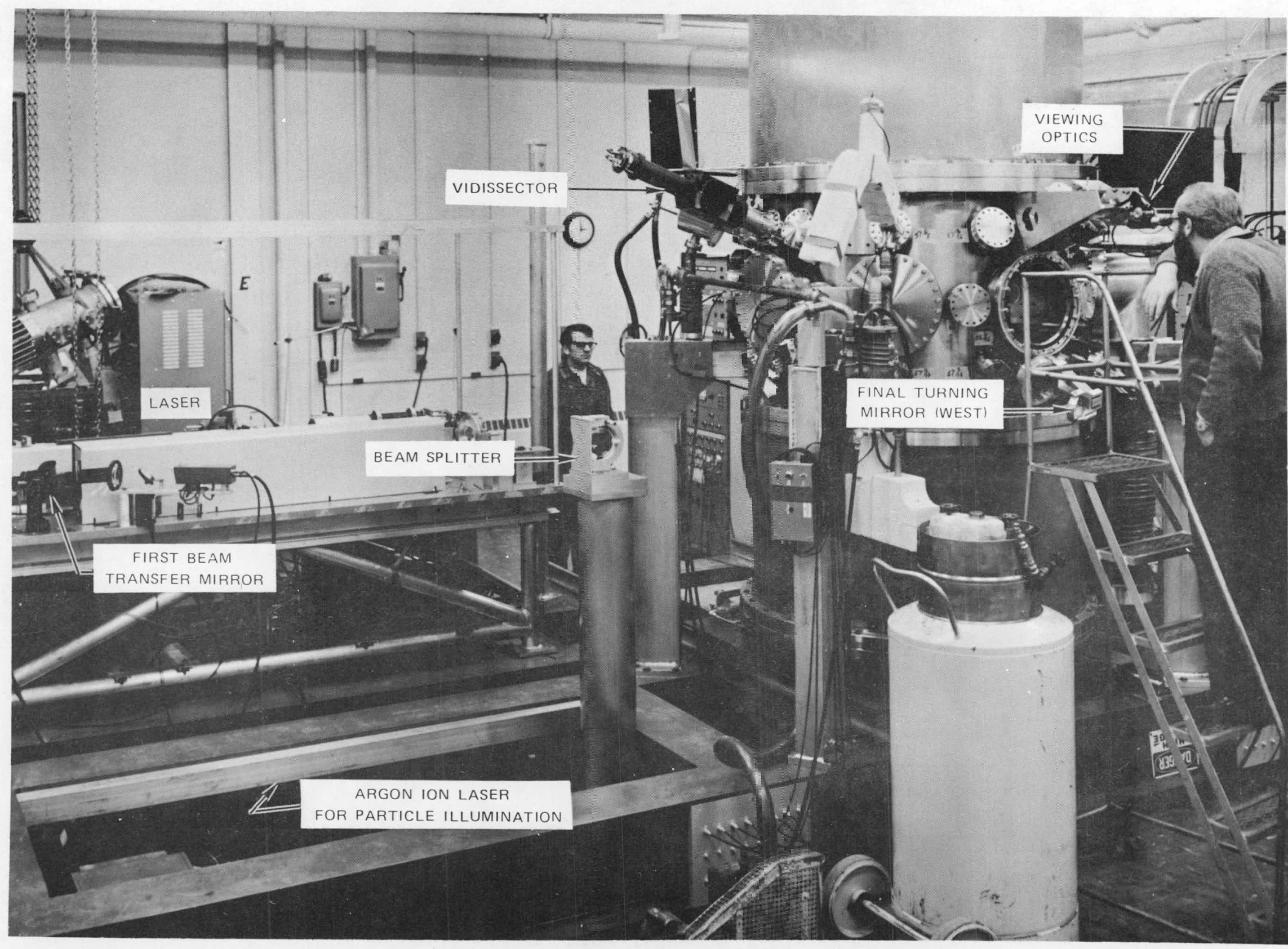




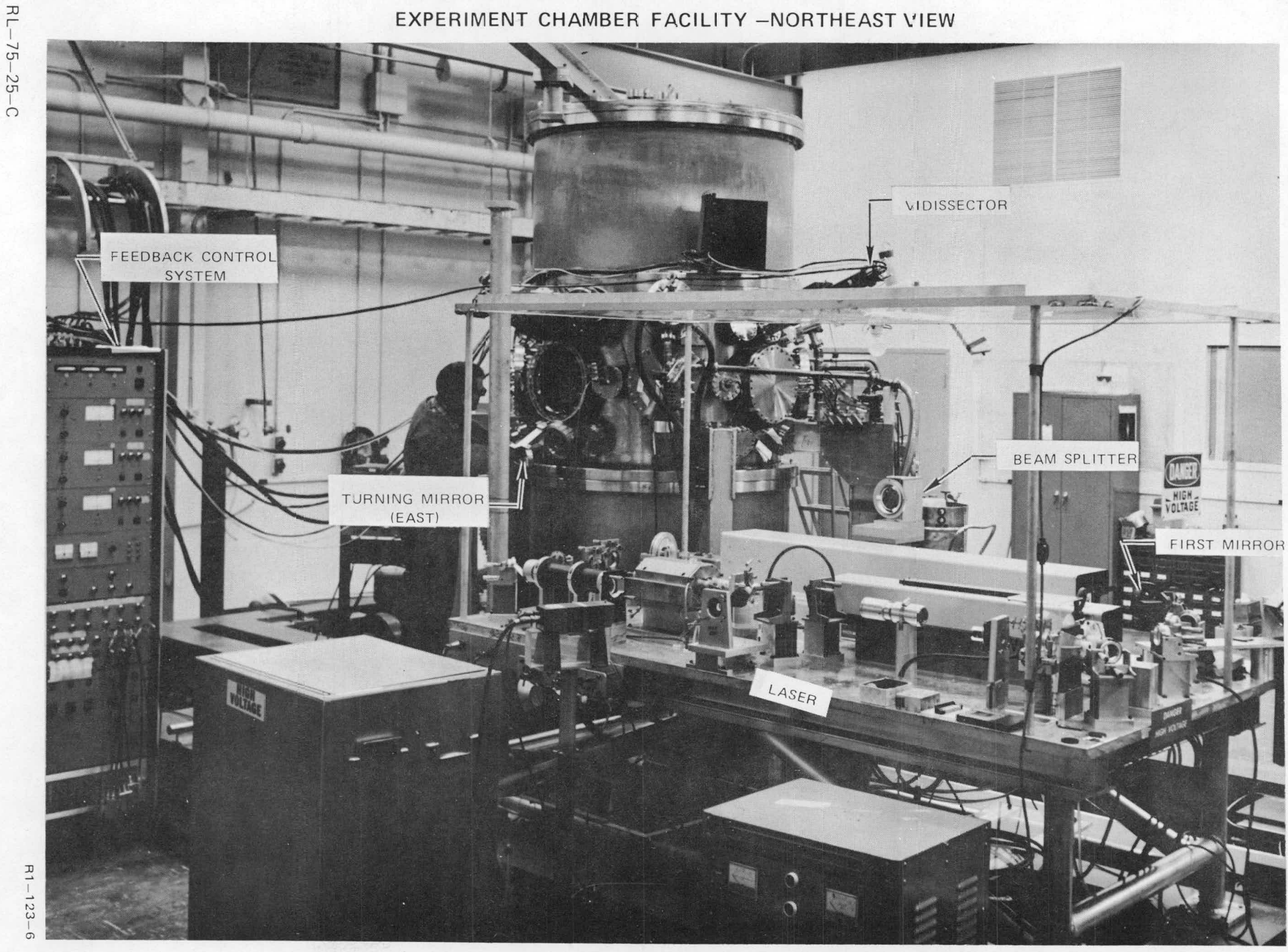




\section{EXPERIMENT CHAMBER FACILITY - SOUTHWEST VIEW}

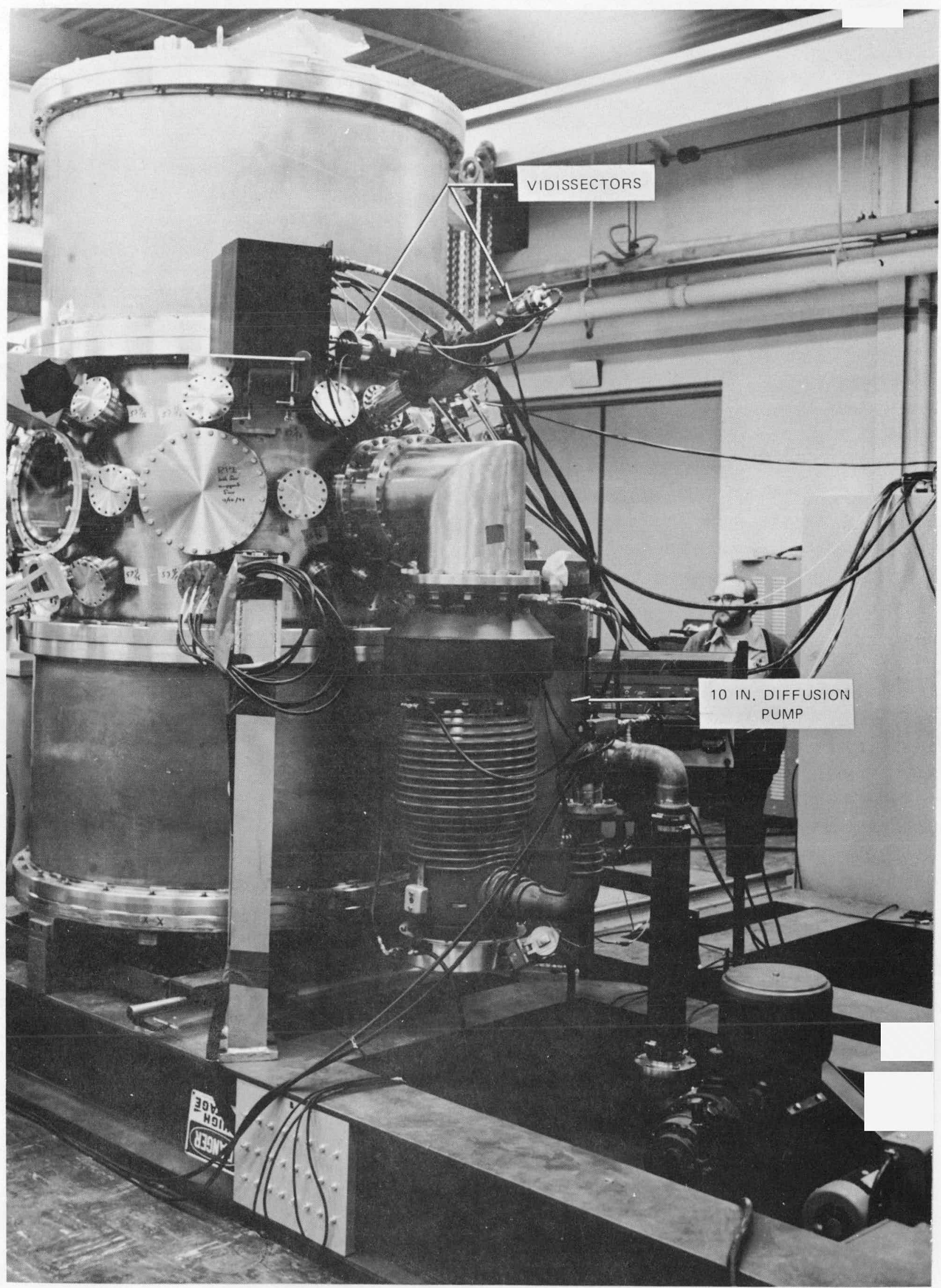




\section{EXPERIMENT CHAMBER INTERIOR -VIEWED FROM ABOVE}

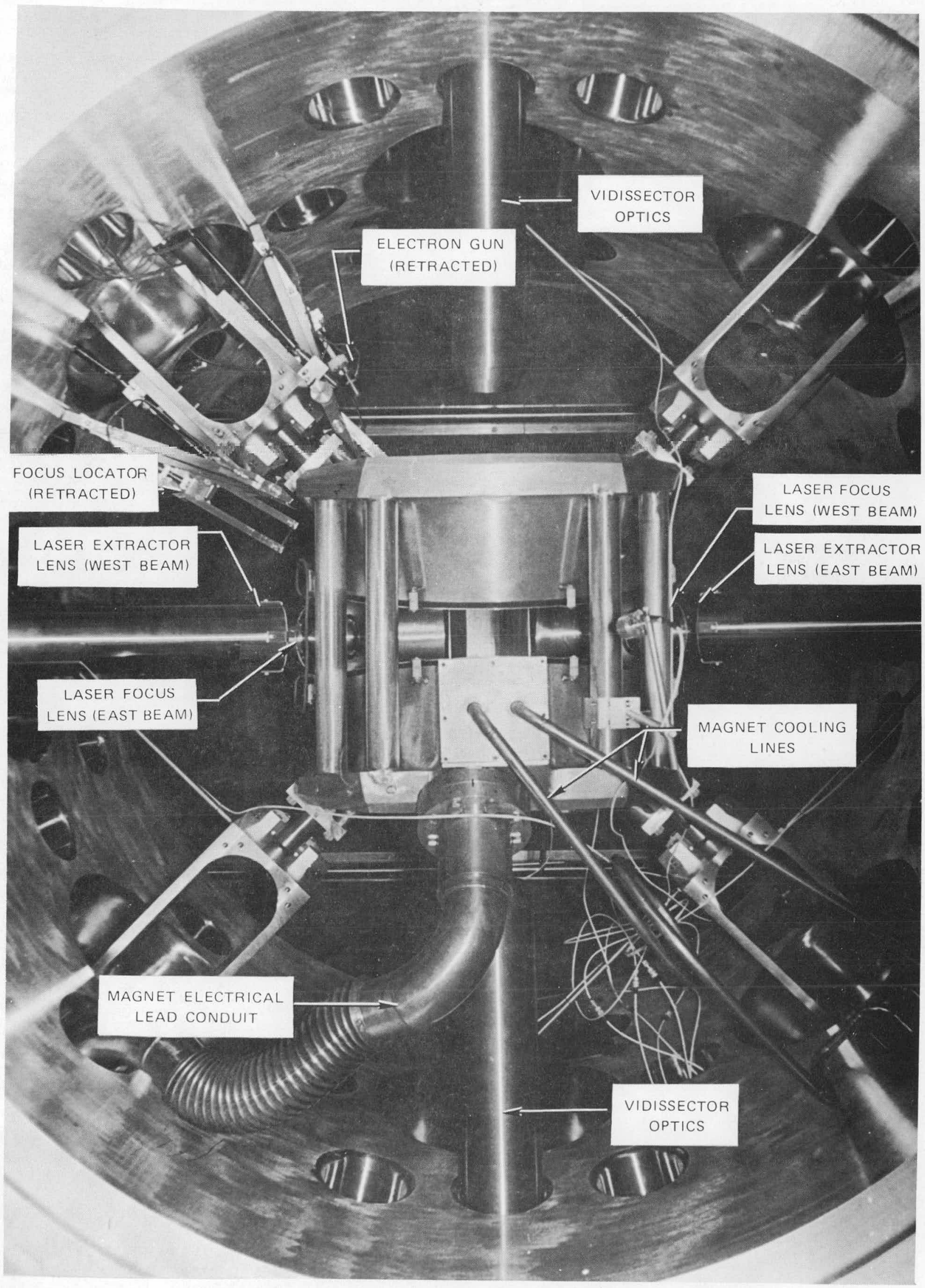




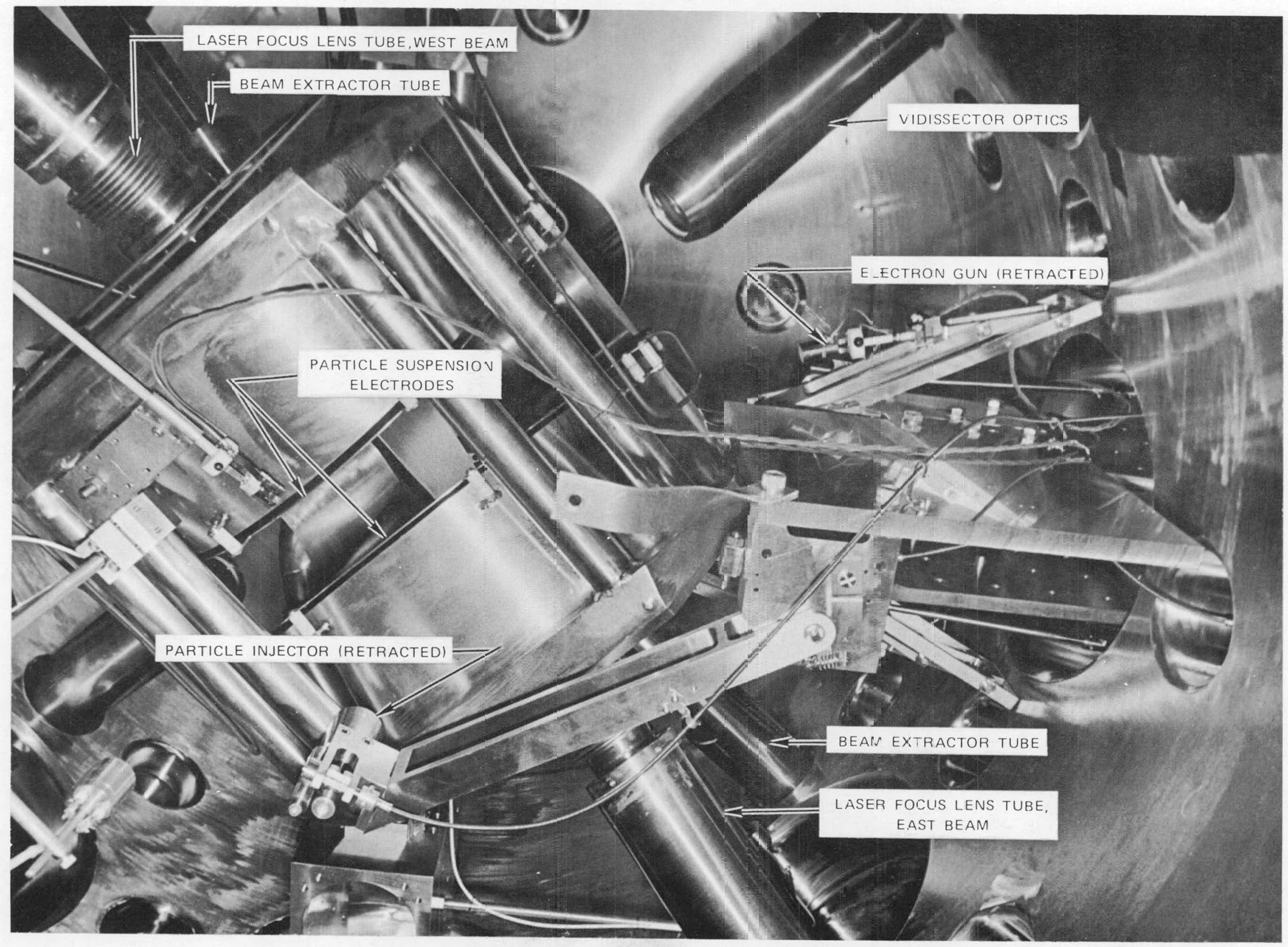




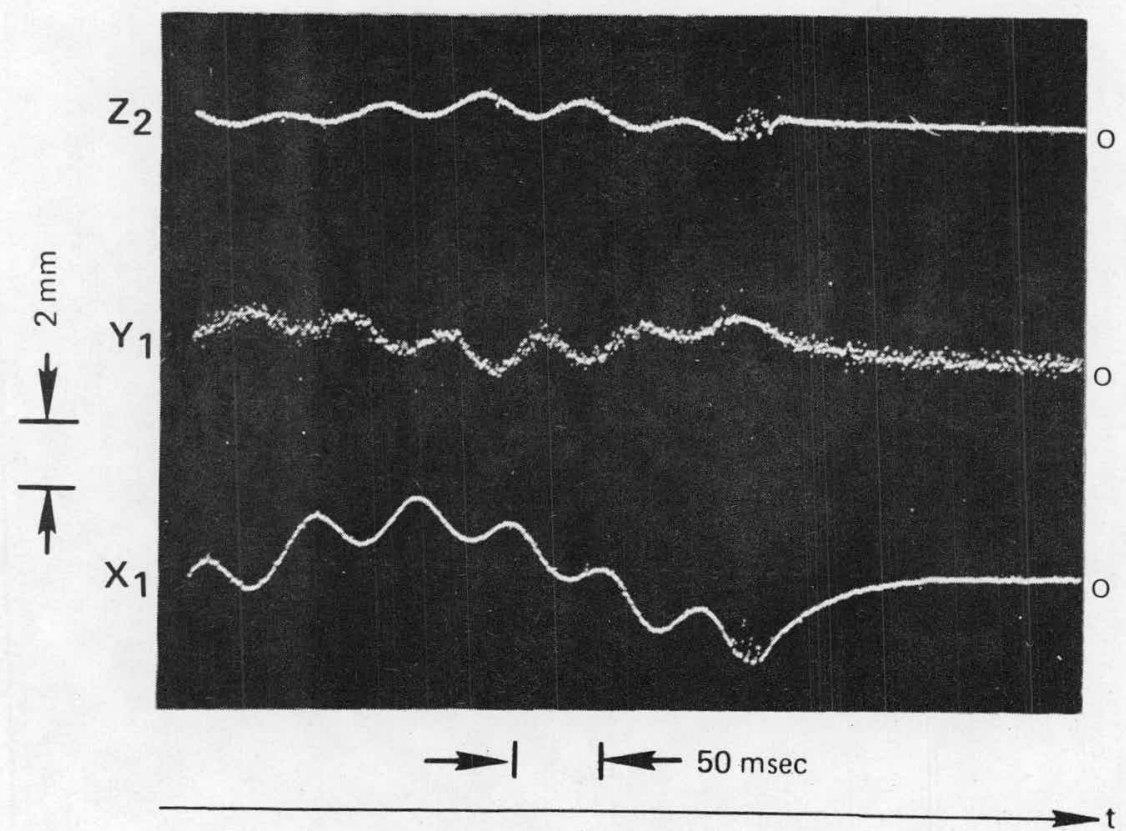




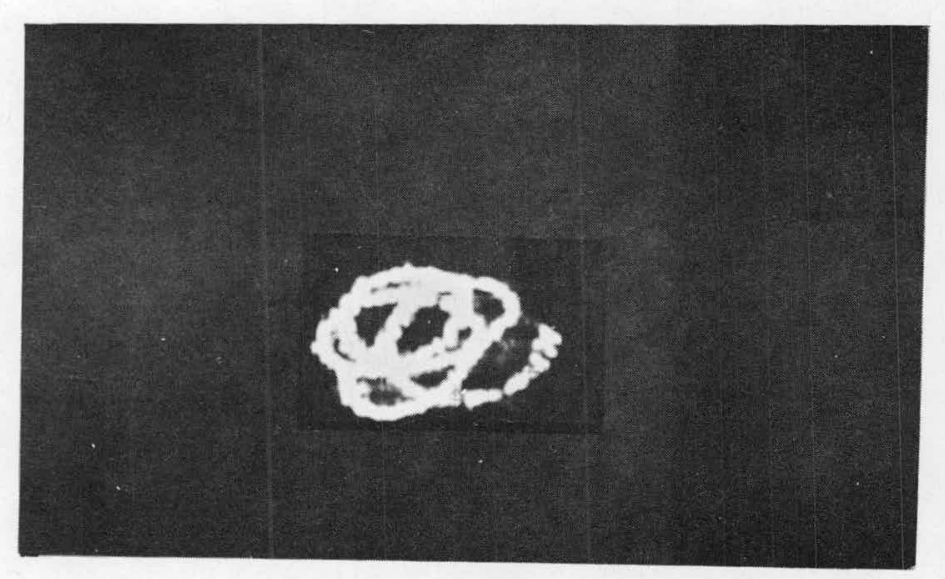

$17 \mathrm{~Hz}$ ac

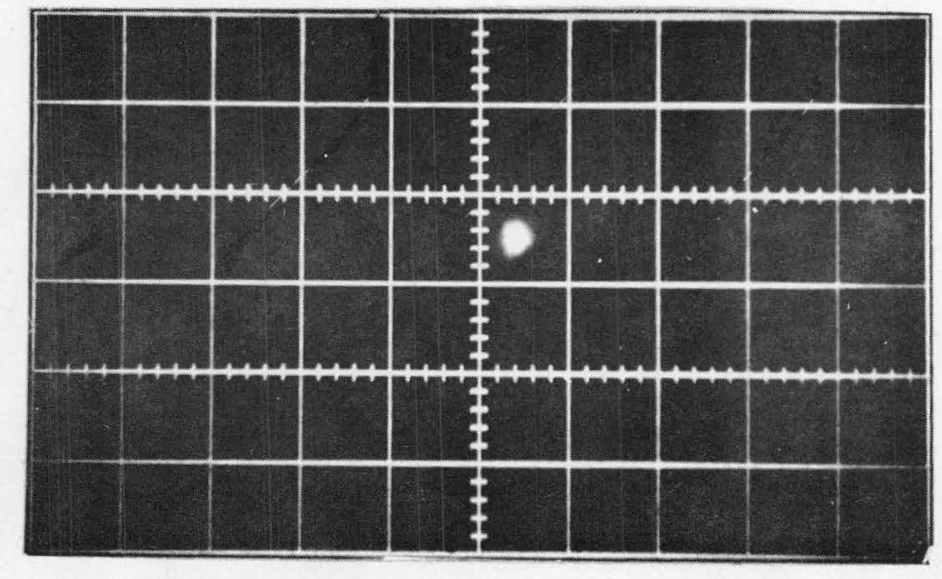

FEEDBACK CONTROLLED 

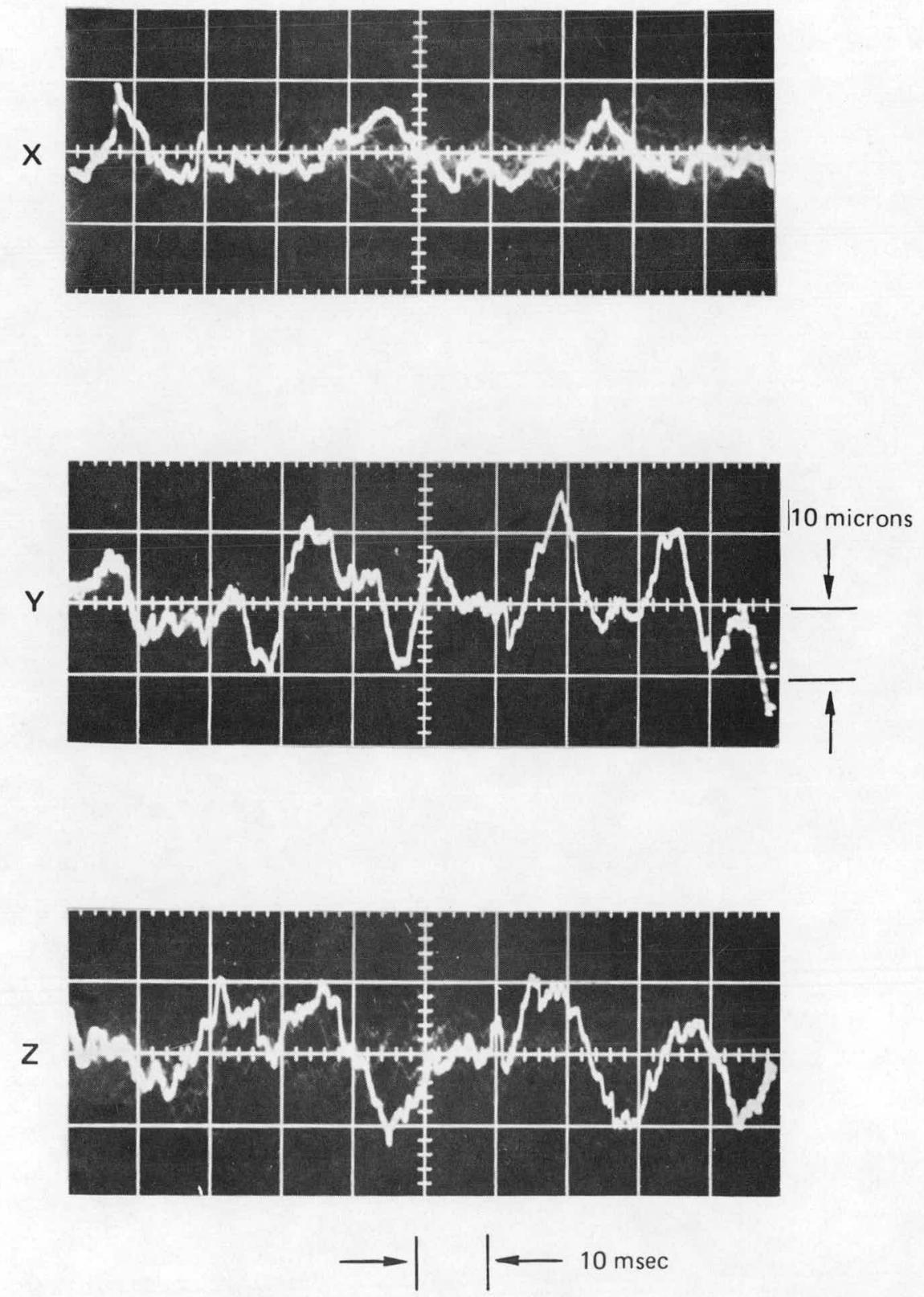
VIDISSECTOR SIGNALS

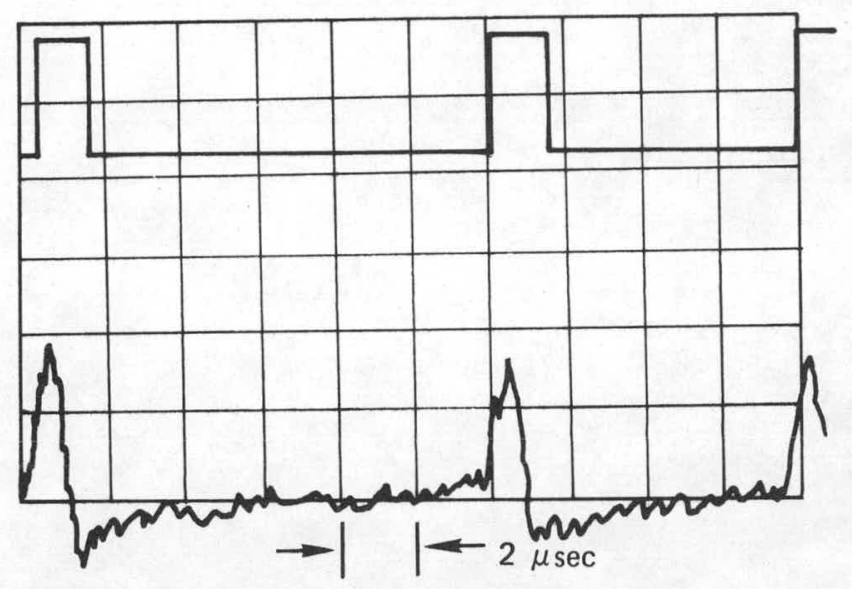

a. VIDISSECTOR SIGNALS AND CORRESPONDING MULTIVIBRATOR PULSES

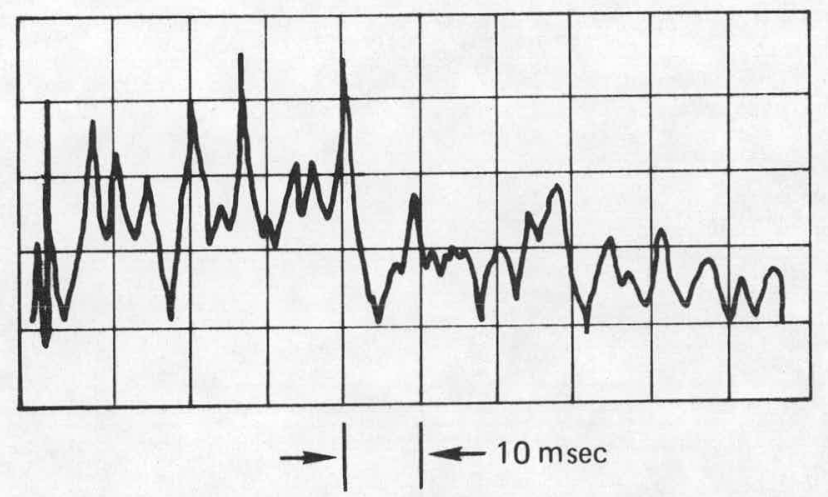

b. MODULATION OF VIDISSECTOR SIGNALS

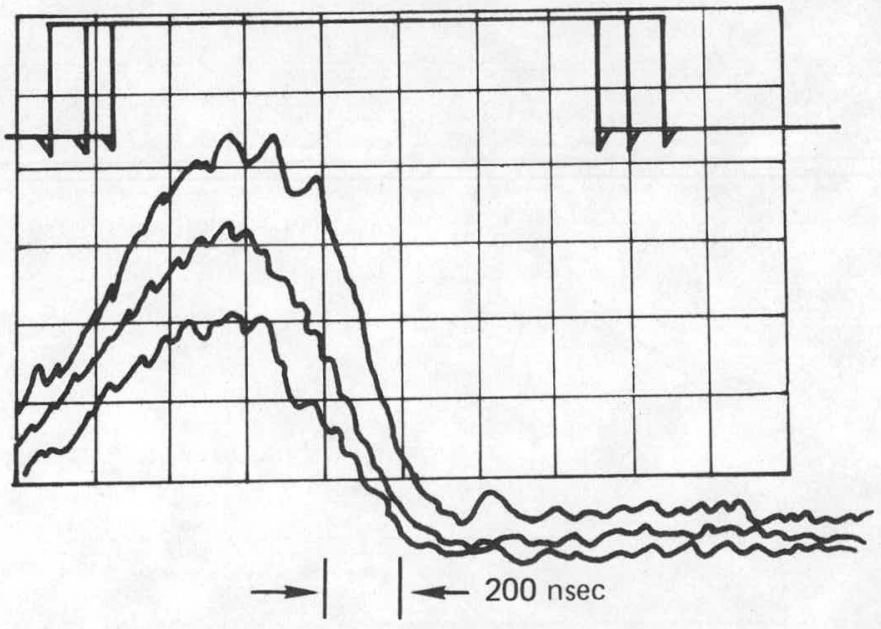

c. VIDISSECTOR SIGNALS ON EXPANDED SWEEP (MULTIPLE TRACE) 
CHARGE COLLECTOR CURRENT - ONE DIRECTION IRRADIATION NO PREPULSE EAST BEAM ONLY
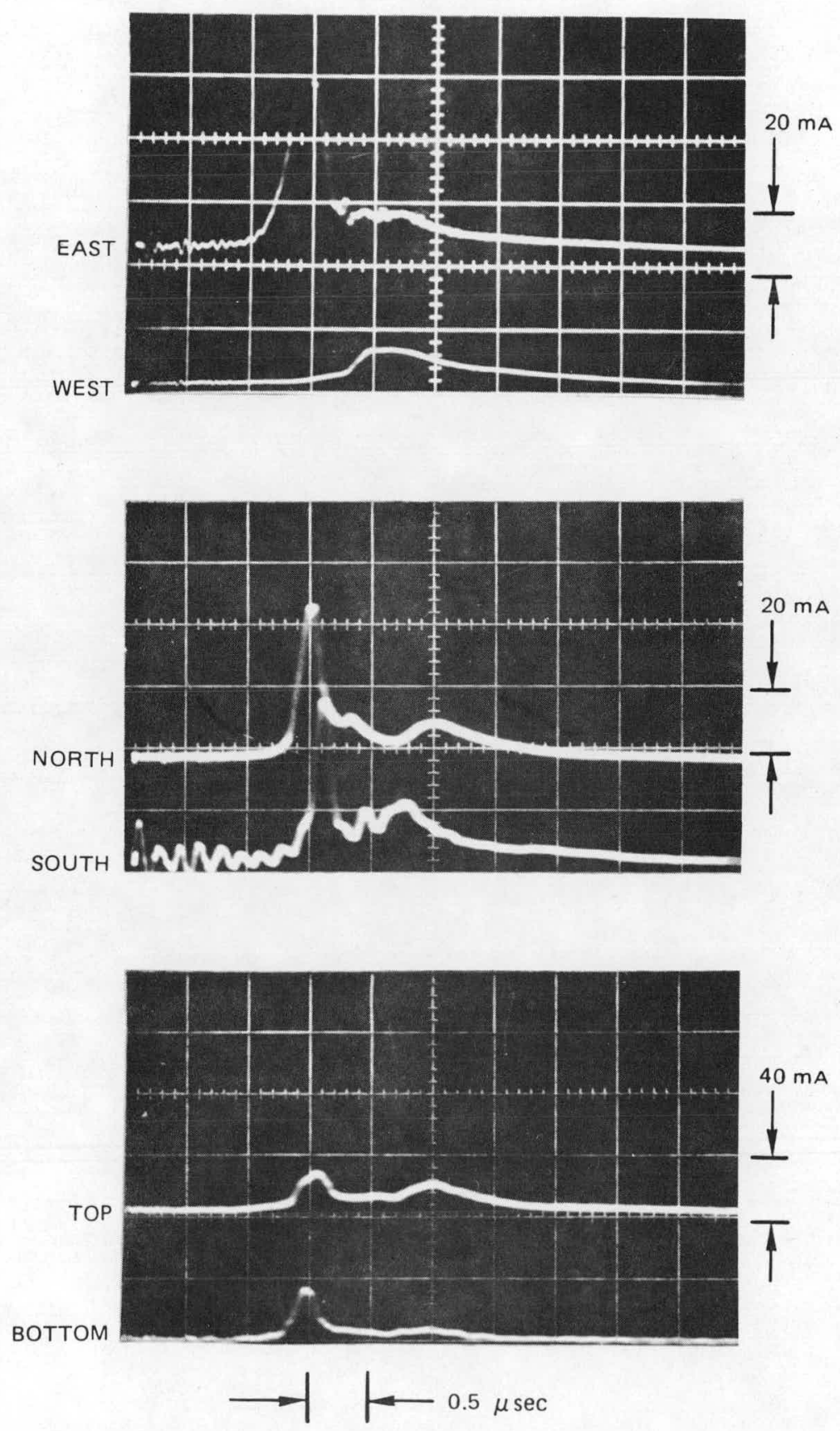
CHARGE COLLECTOR CURRENT - ONE DIRECTION IRRADIATION NO PREPULSE WEST BEAM ONLY
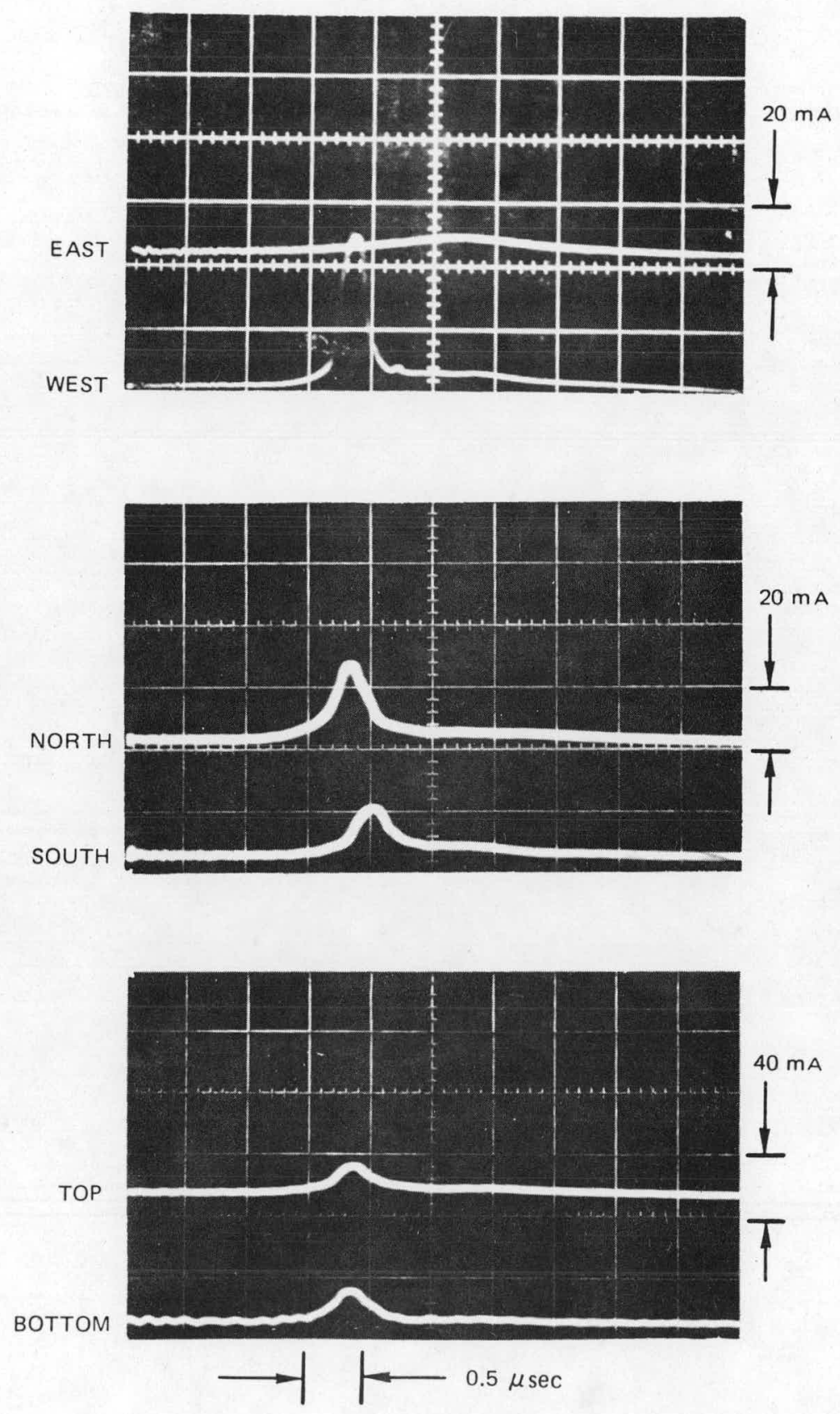


\section{CHARGE COLLECTOR CURRENT - TWO DIRECTION IRRADIATION} NO PREPULSE
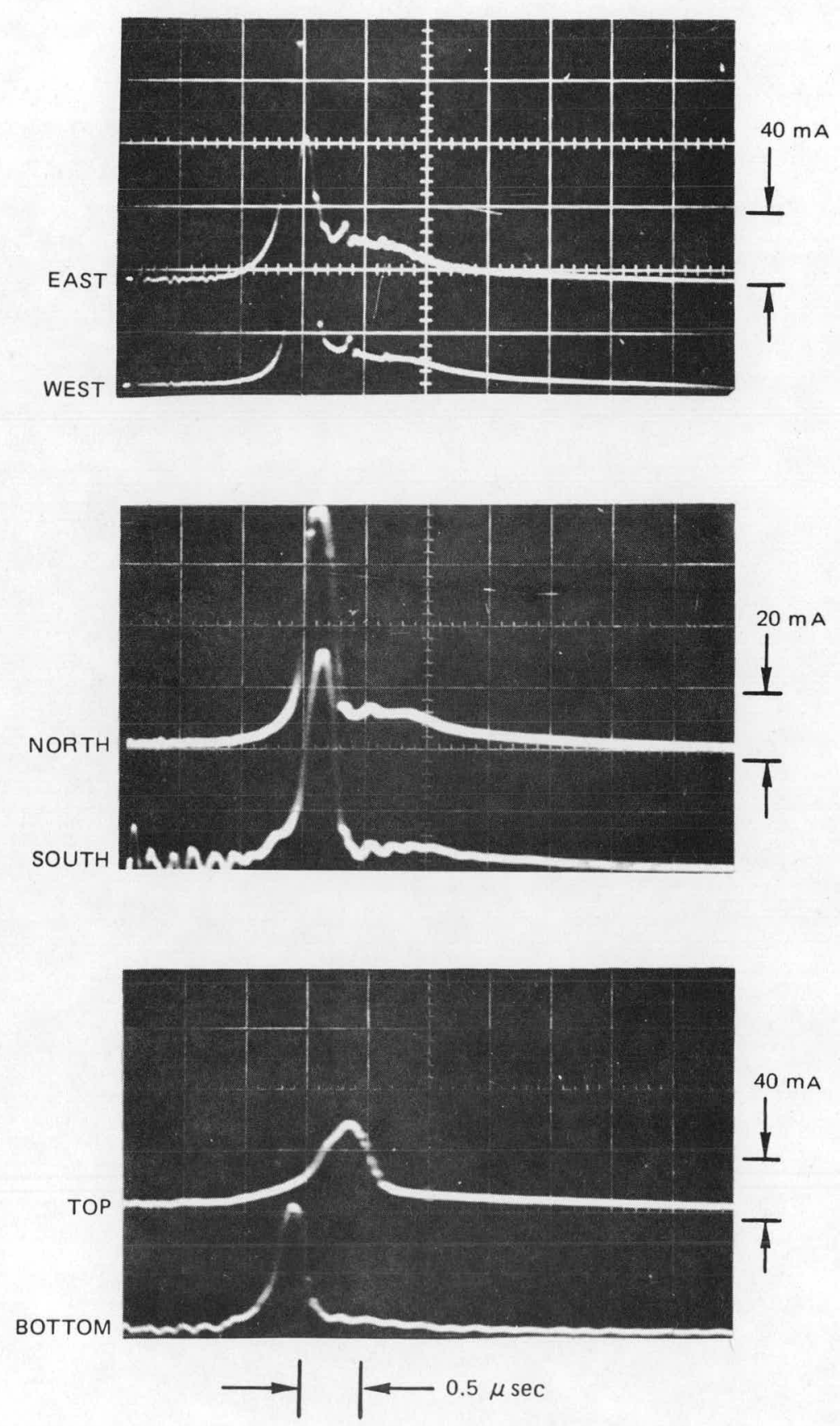


\section{CHARGE COLLECTOR CURRENT - TWO DIRECTION IRRADIATION} MAXIMUM PREPULSE
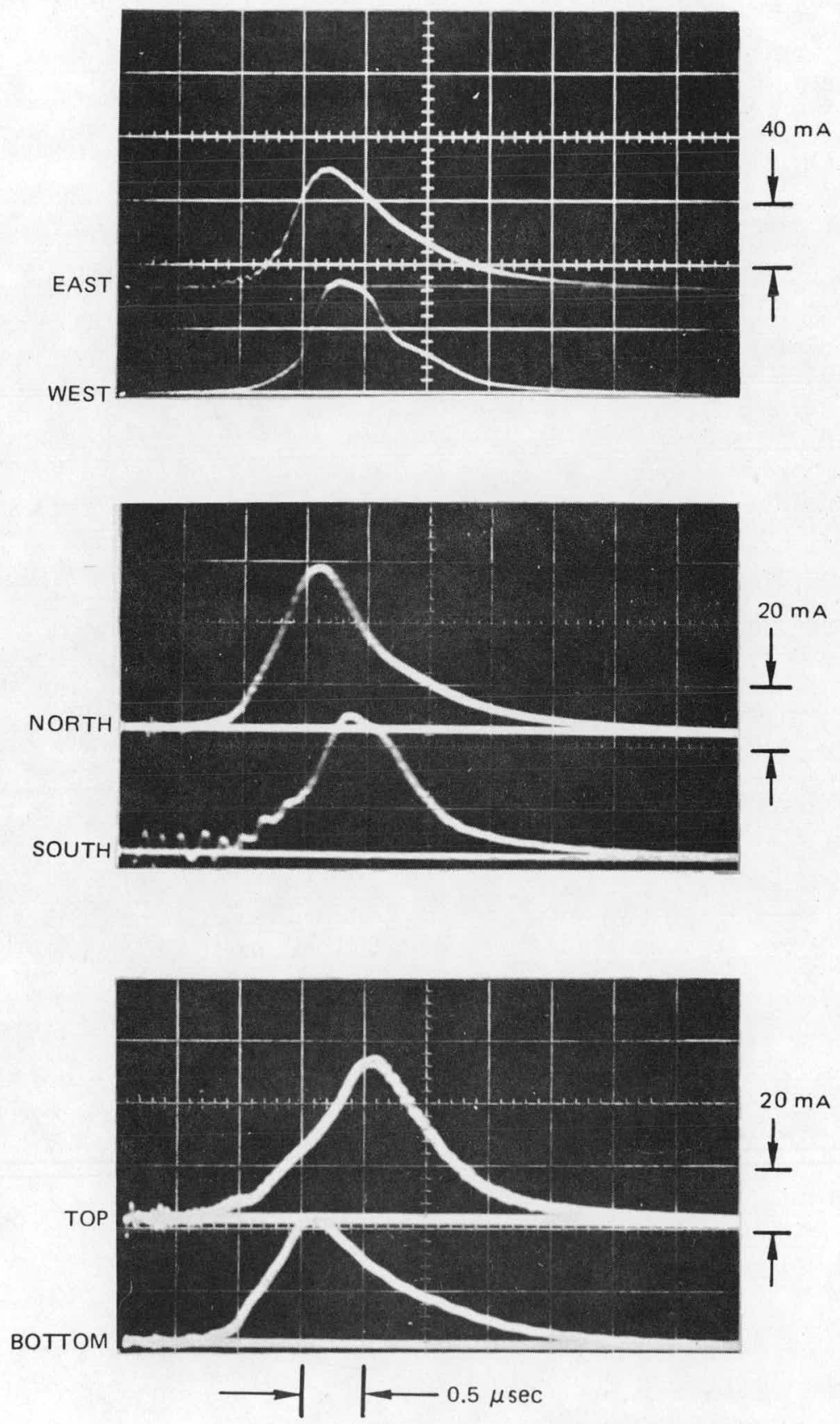
FIG. 15

\section{EFFECT OF PREPULSE ON PLASMA ENERGY}

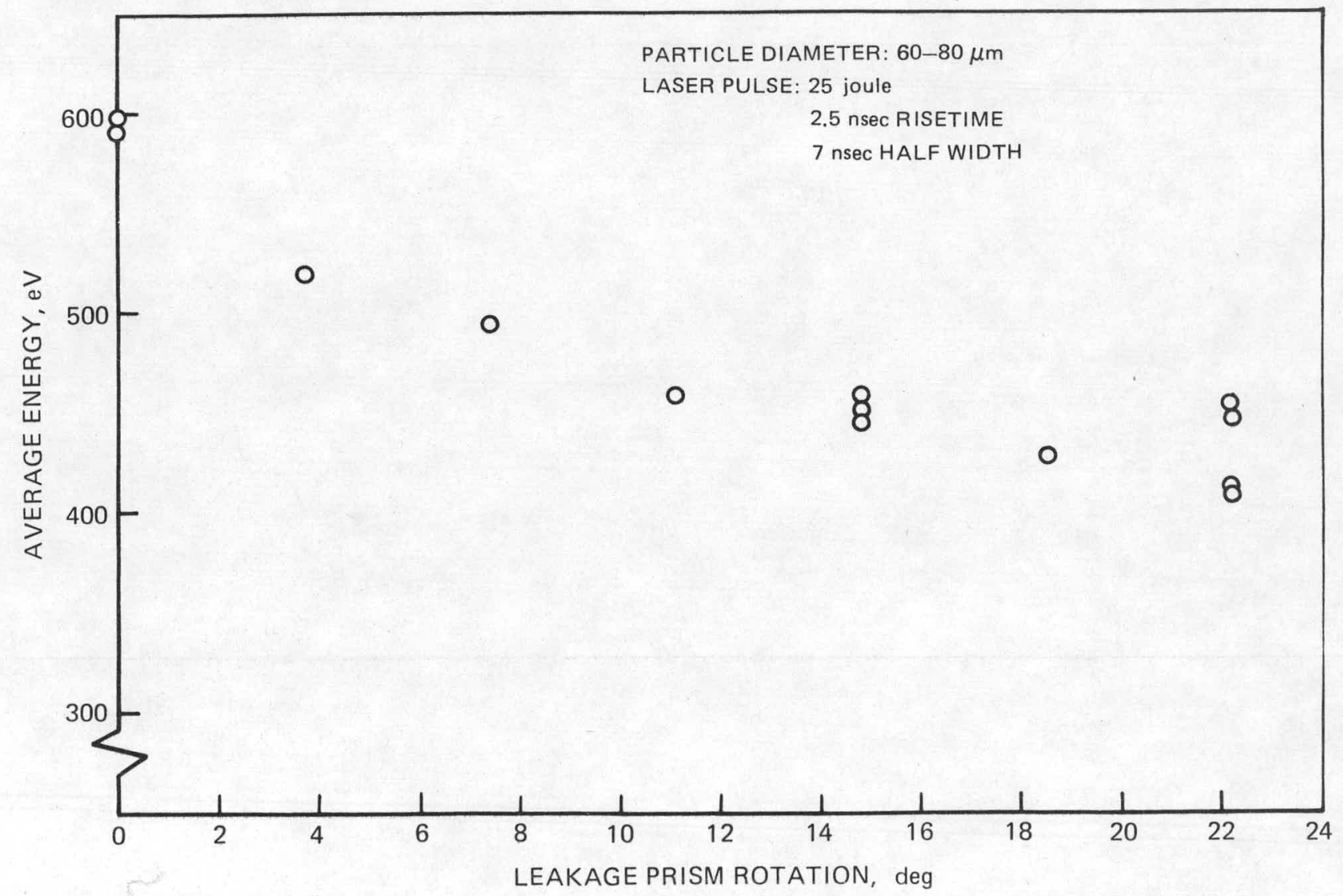


ELEVATION VIEW OF LITE ENERGETIC NEUTRAL BEAM LINE FOR BEAM EVALUATION WITHOUT EXPERIMENT CHAMBER

$$
\text { ニニニ LIQUID N } 2 \text { DEWARS }
$$

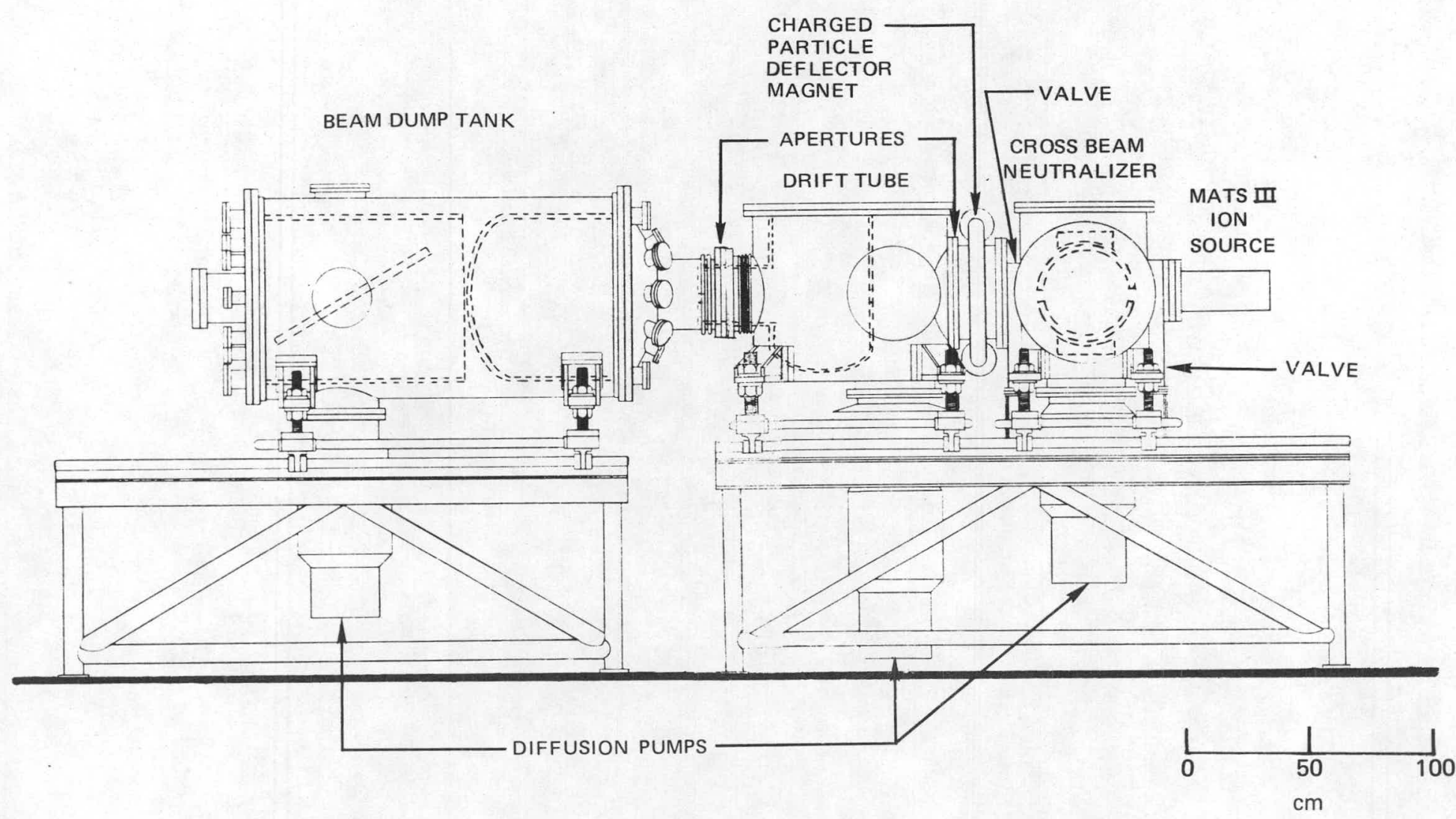


LITE NEUTRAL BEAM LINE WITHOUT EXPERIMENT CHAMBER

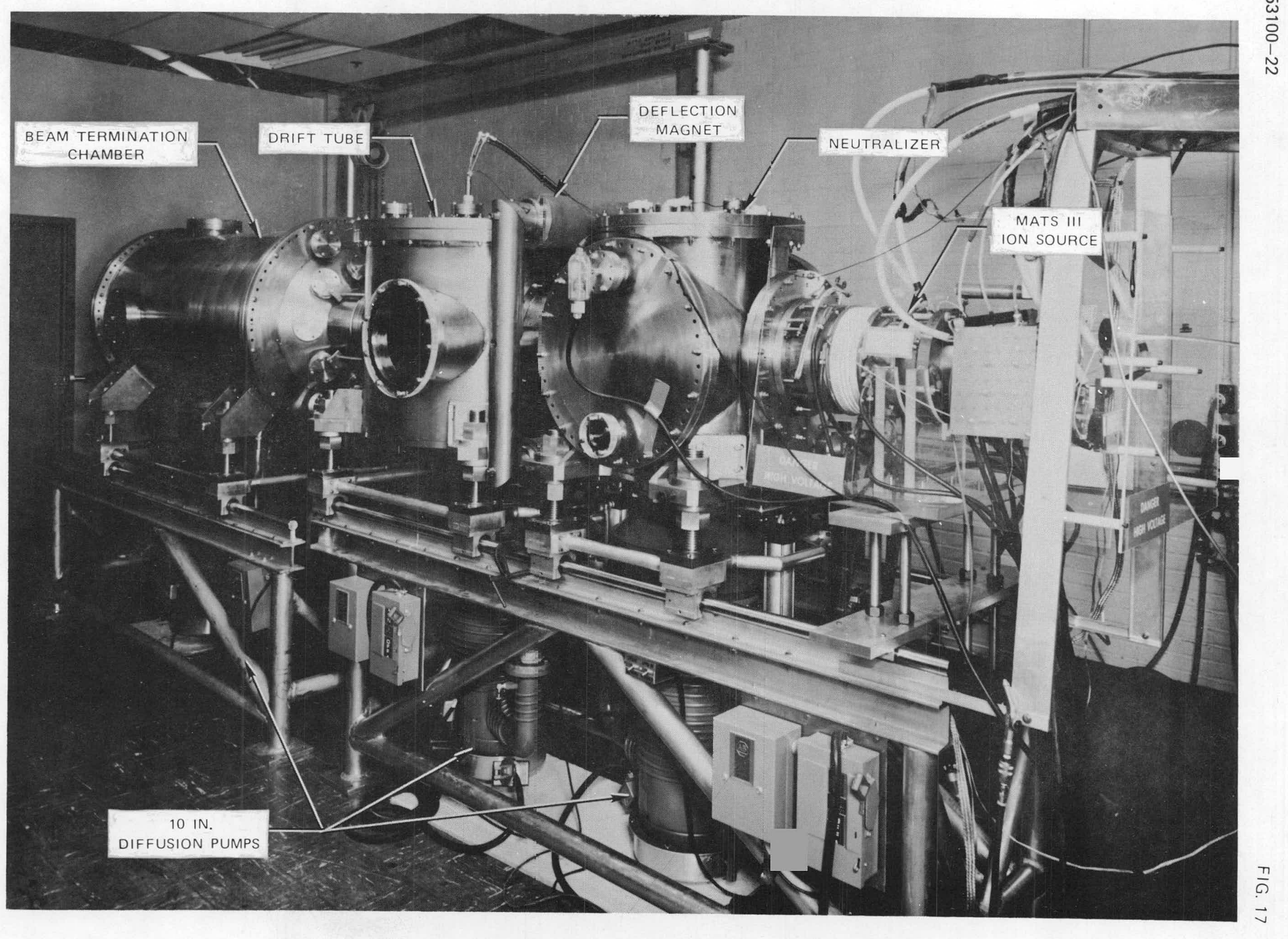




\section{MEASURED PERFORMANCE OF MATS-III ION SOURCE - UARL COPY}

O TESTED AT LLL; $100 \mathrm{~cm}^{2}$ TARGET, $225 \mathrm{~cm}$ FROM SOURCE

$\triangle$ TESTED AT UARL; $182 \mathrm{~cm}^{2}$ TARGET, $170 \mathrm{~cm}$ FROM SOURCE, CORRECTED FOR $75 \%$ ELECTRODE APERTURING

BEAM CURRENT DETERMINED CALORIMETRICALLY

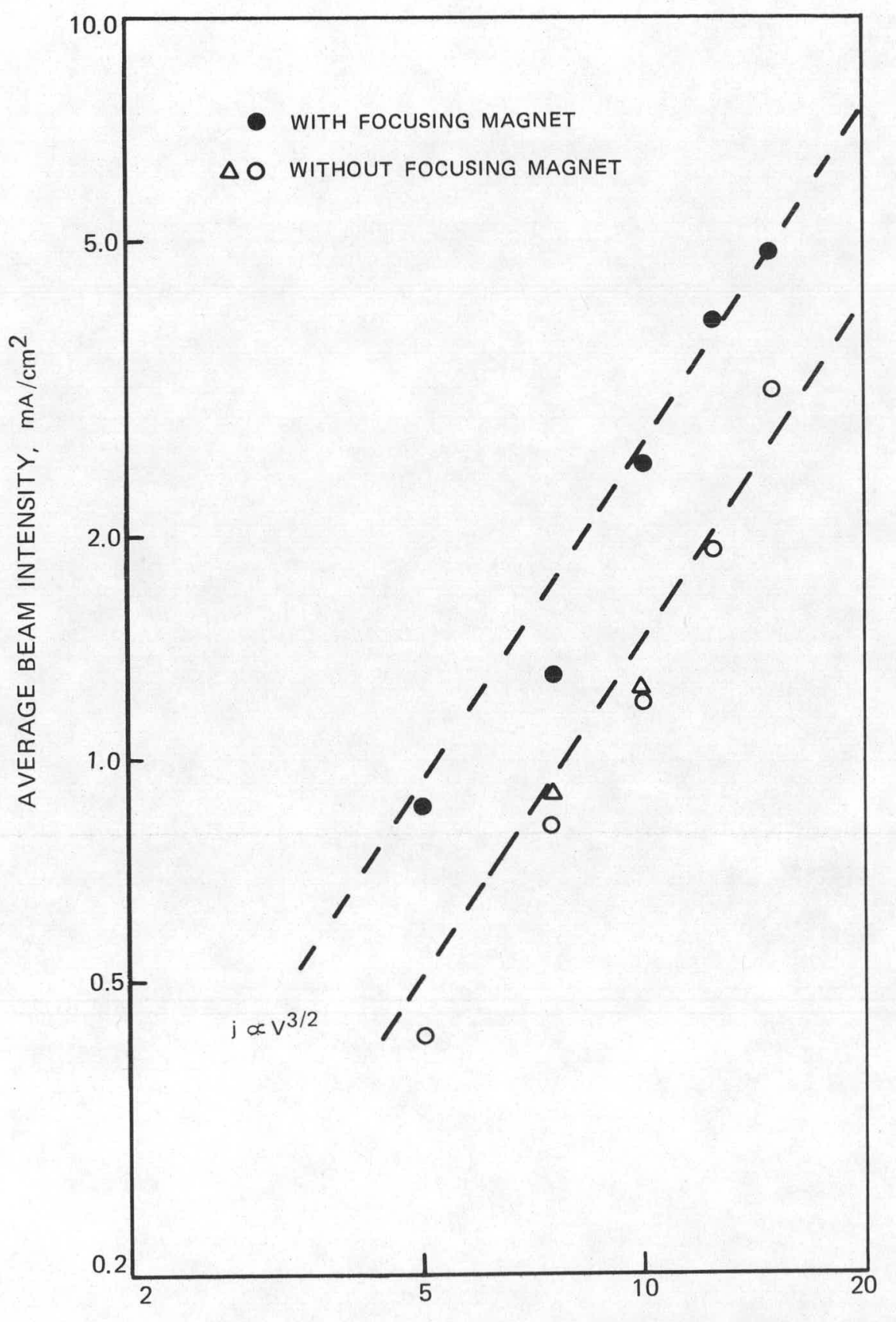

ACCELERATION POTENTIAL, kV 


\section{ELEVATION VIEW OF ION SOURCE COUPLED TO BEAM TERMINATION CHAMBER}

EXPERIMENTAL SET-UP FOR INITIAL ION SOURCE TESTS
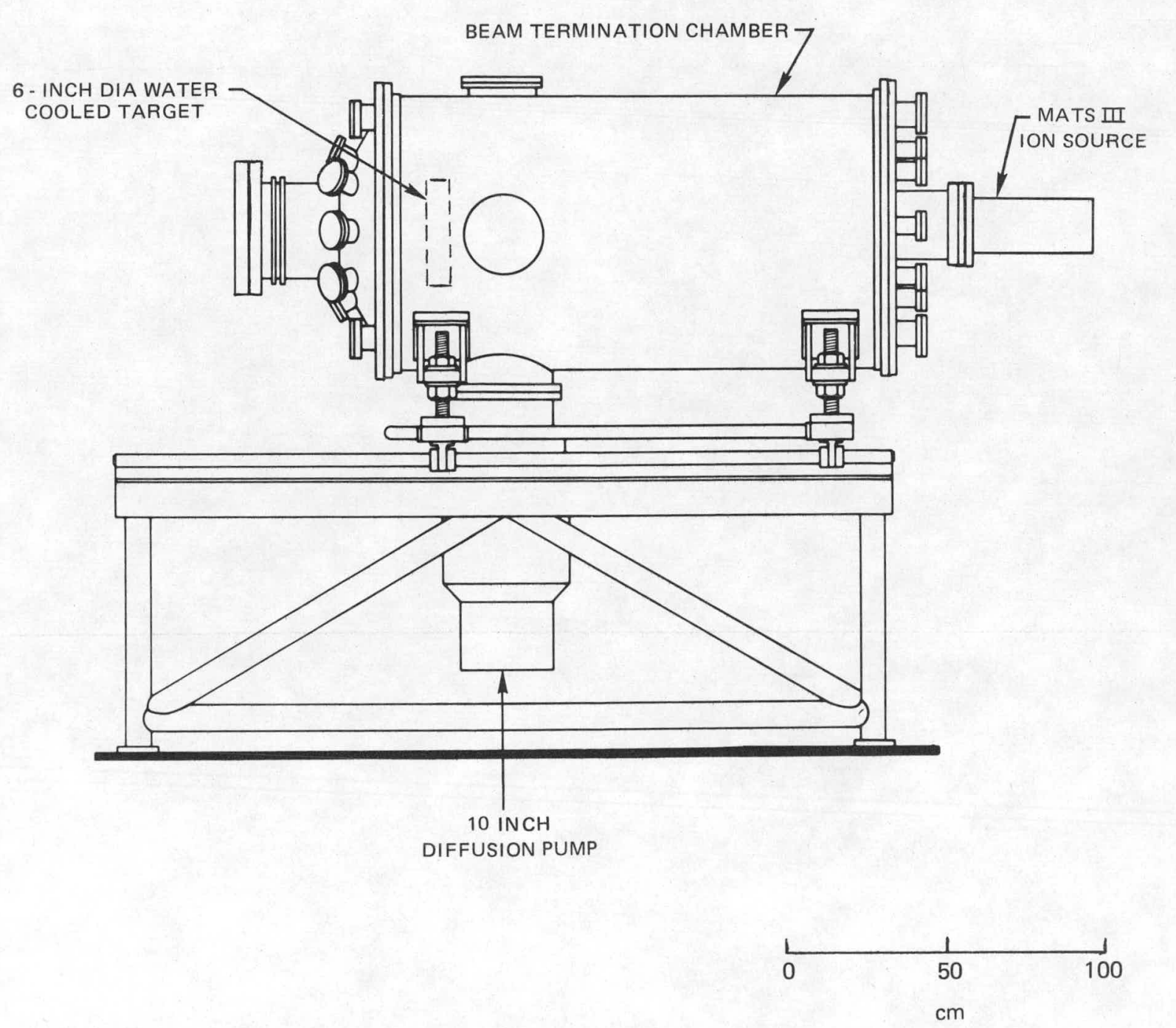


\section{TIME DEPENDENT RESPONSE OF ARC POWER SUPPLY AND ION BEAM}

ACCELERATING VOLTAGE $\simeq 8 \mathrm{kV}$ (UNSWITCHED)

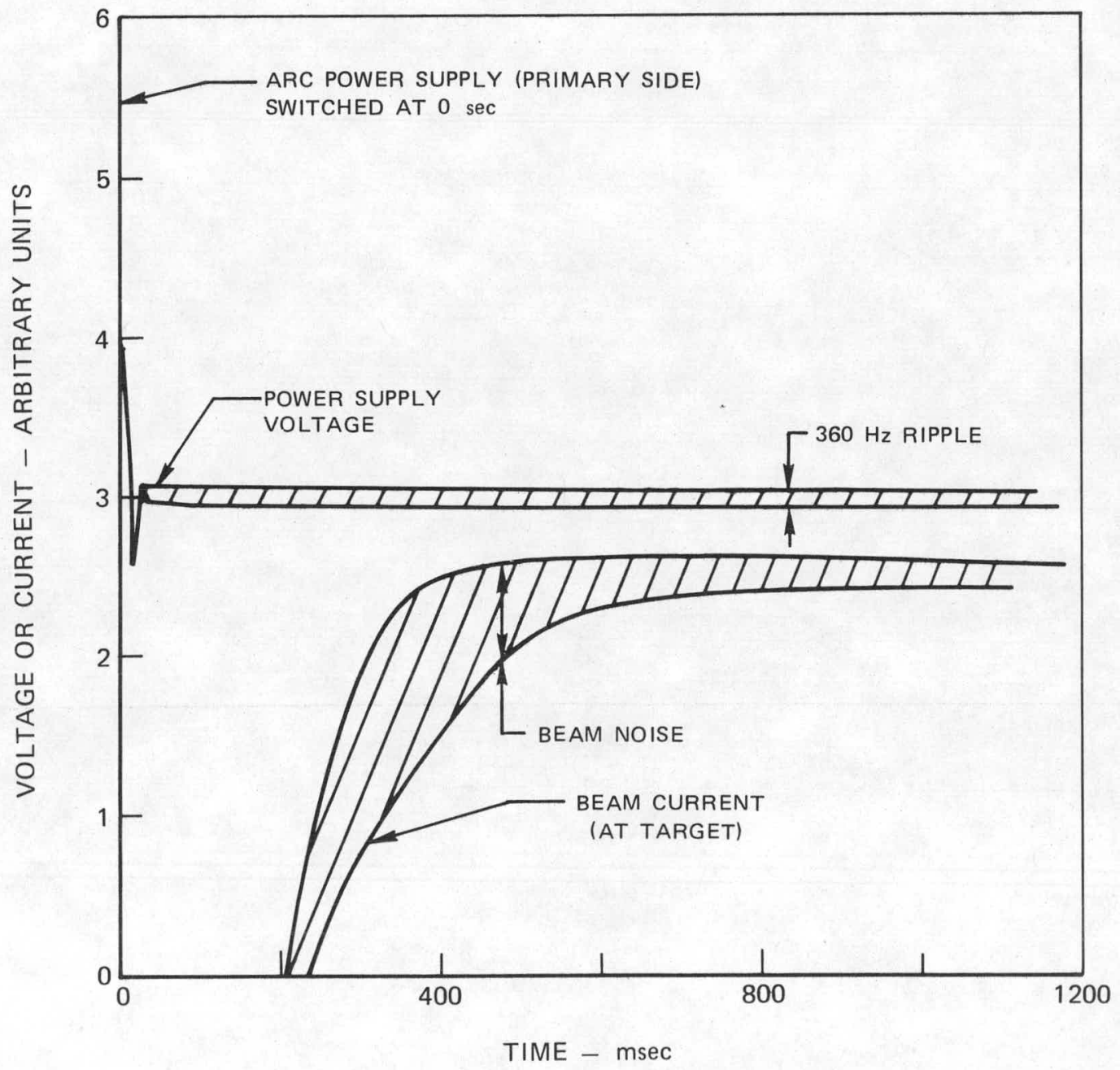




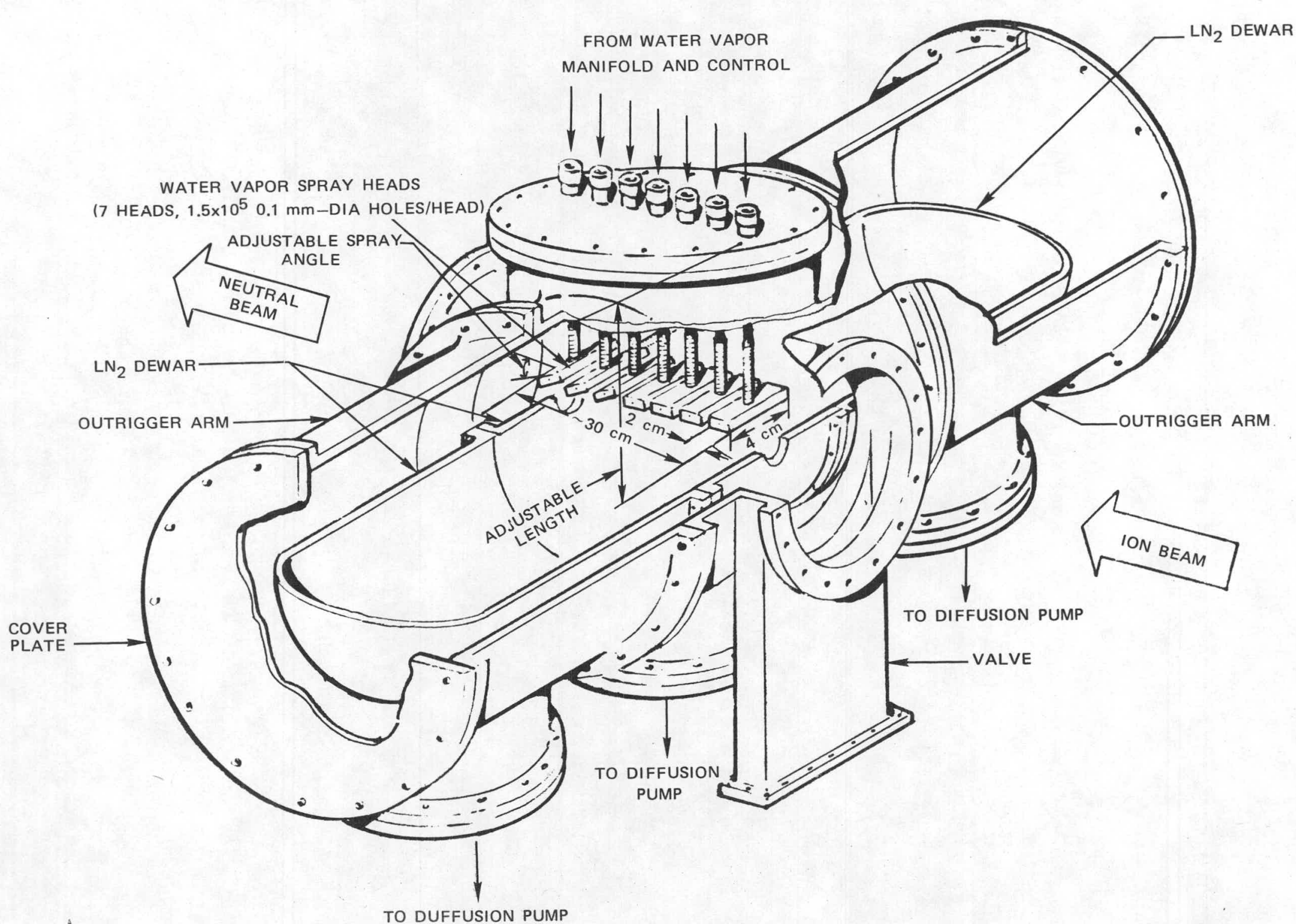


ASSEMBLED NEUTRALIZER SPRAY HEADS AND DEWARS

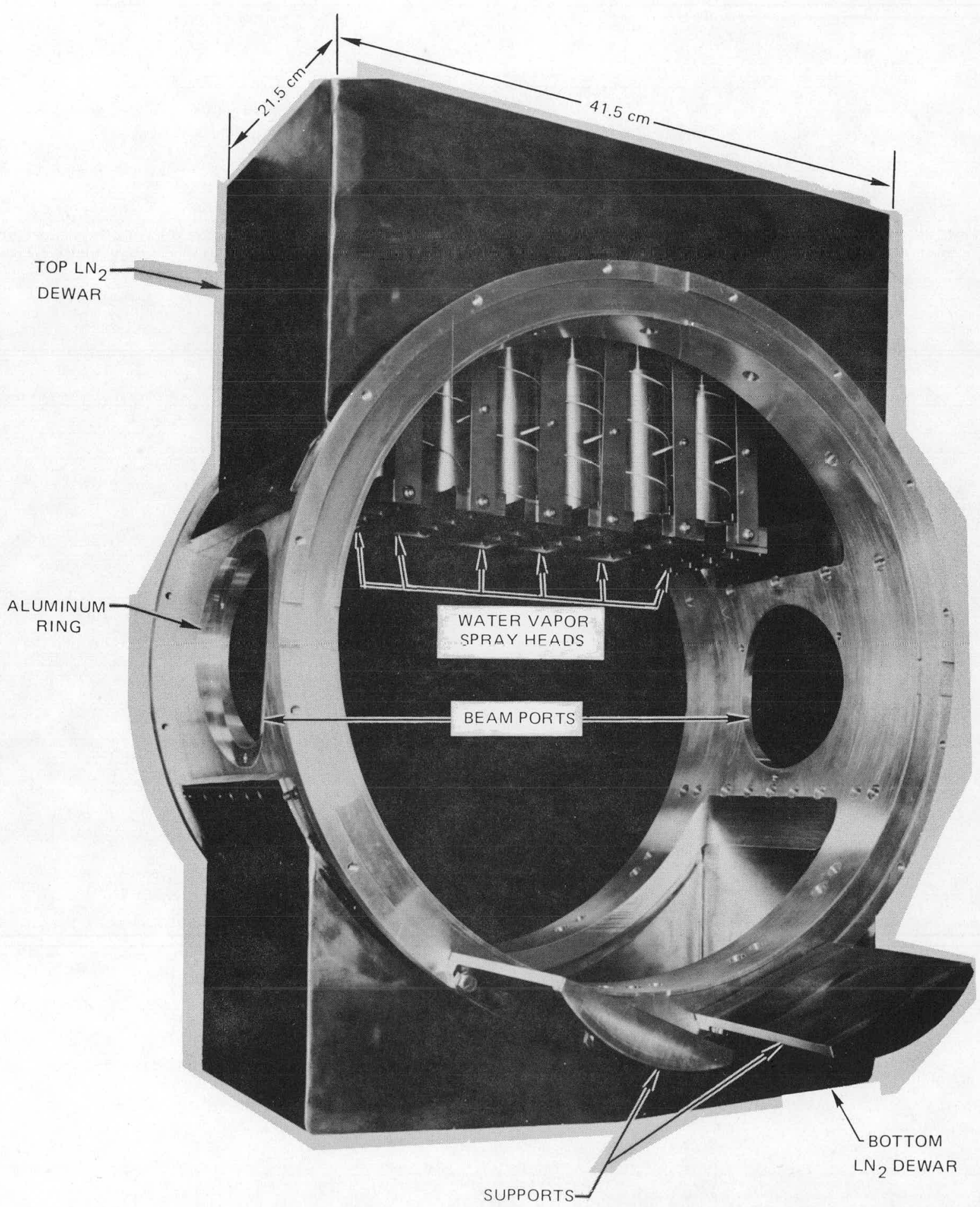




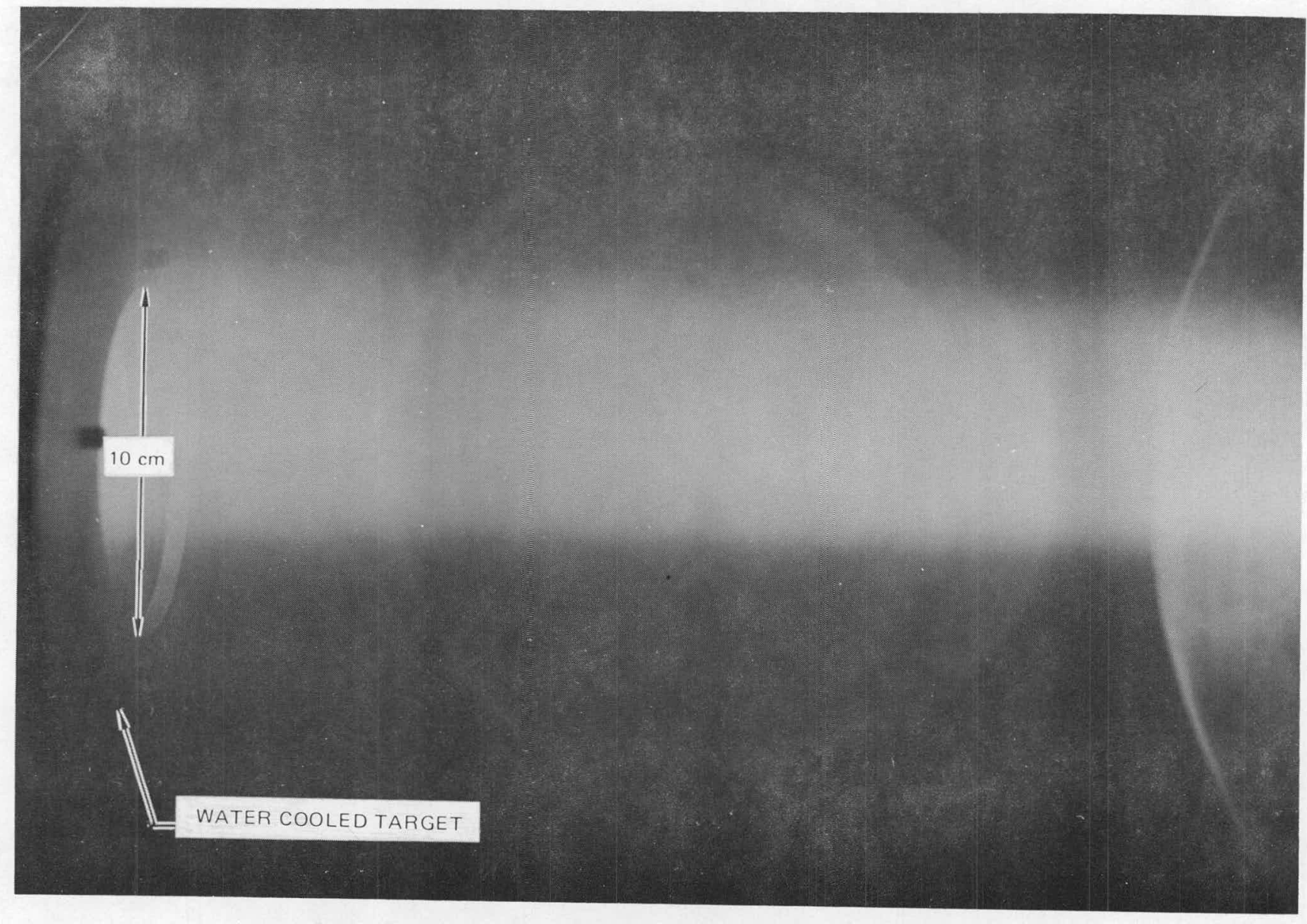




\section{BEAM DEFLECTED BY MAGNET}

VIEW AT 45 DEG FROM BEAM, TOWARD DEFLECTION MAGNET

ACCELERATING VOLTAGE $9 \mathrm{kV}$

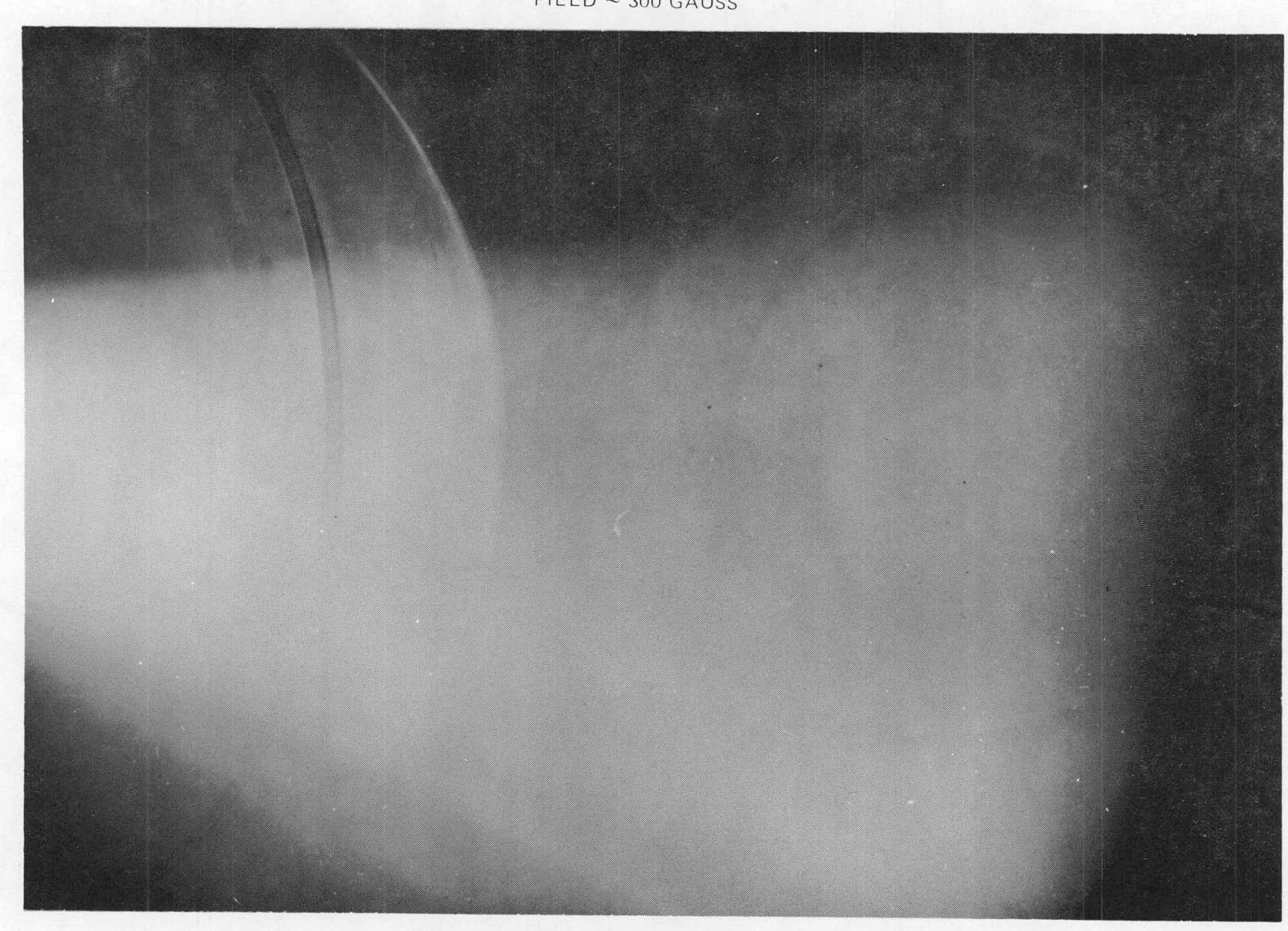


SCHEMATIC OF FAST ATOM ENERGY ANALYSER

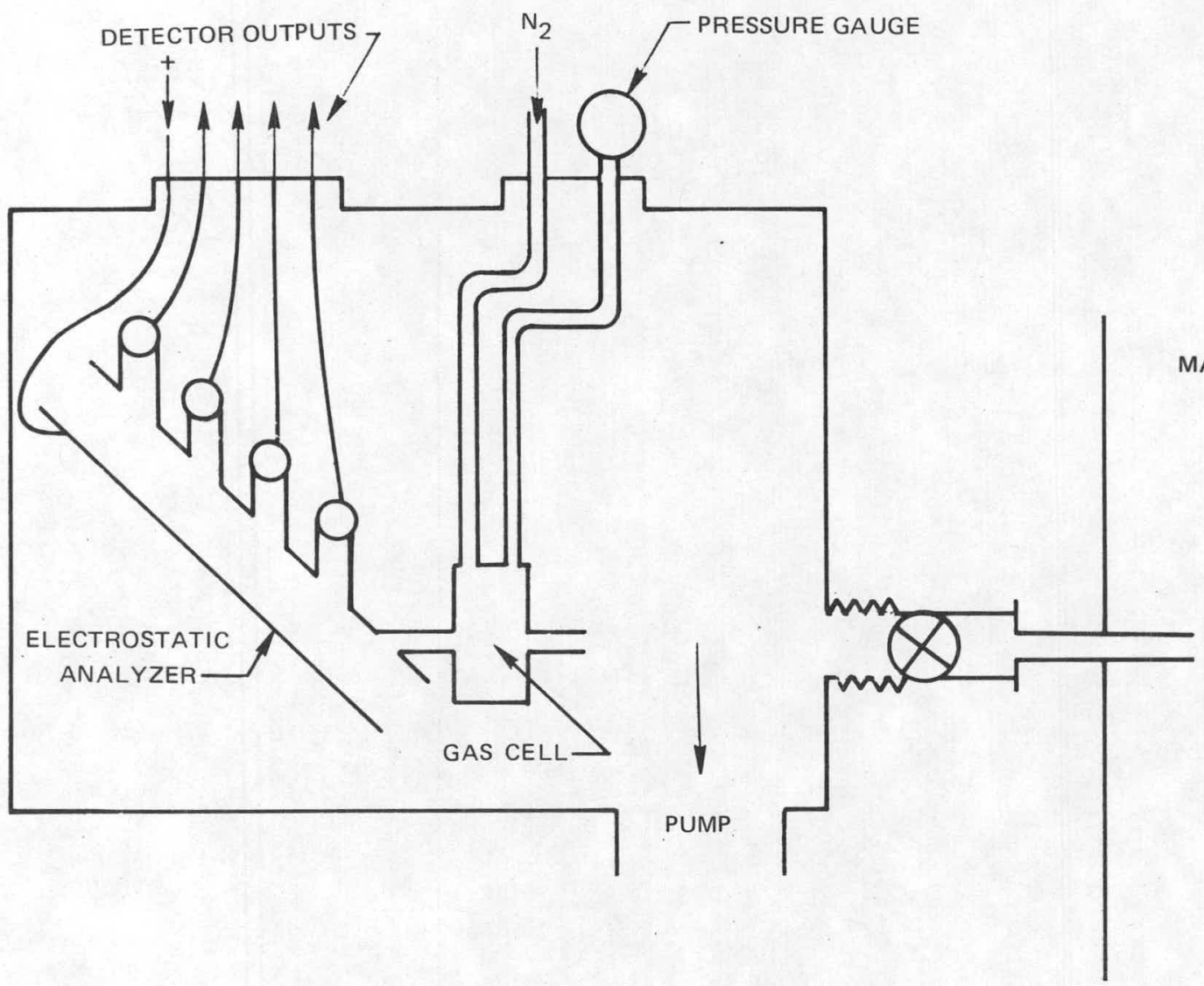

MAIN VACUUM SYSTEM

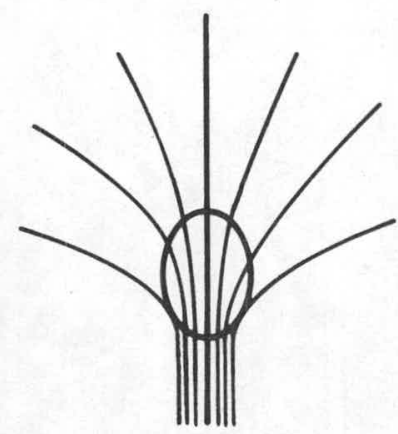


BLOCK DIAGRAM OF FAST ATOM ENERGY ANALYSER ELECTRONICS

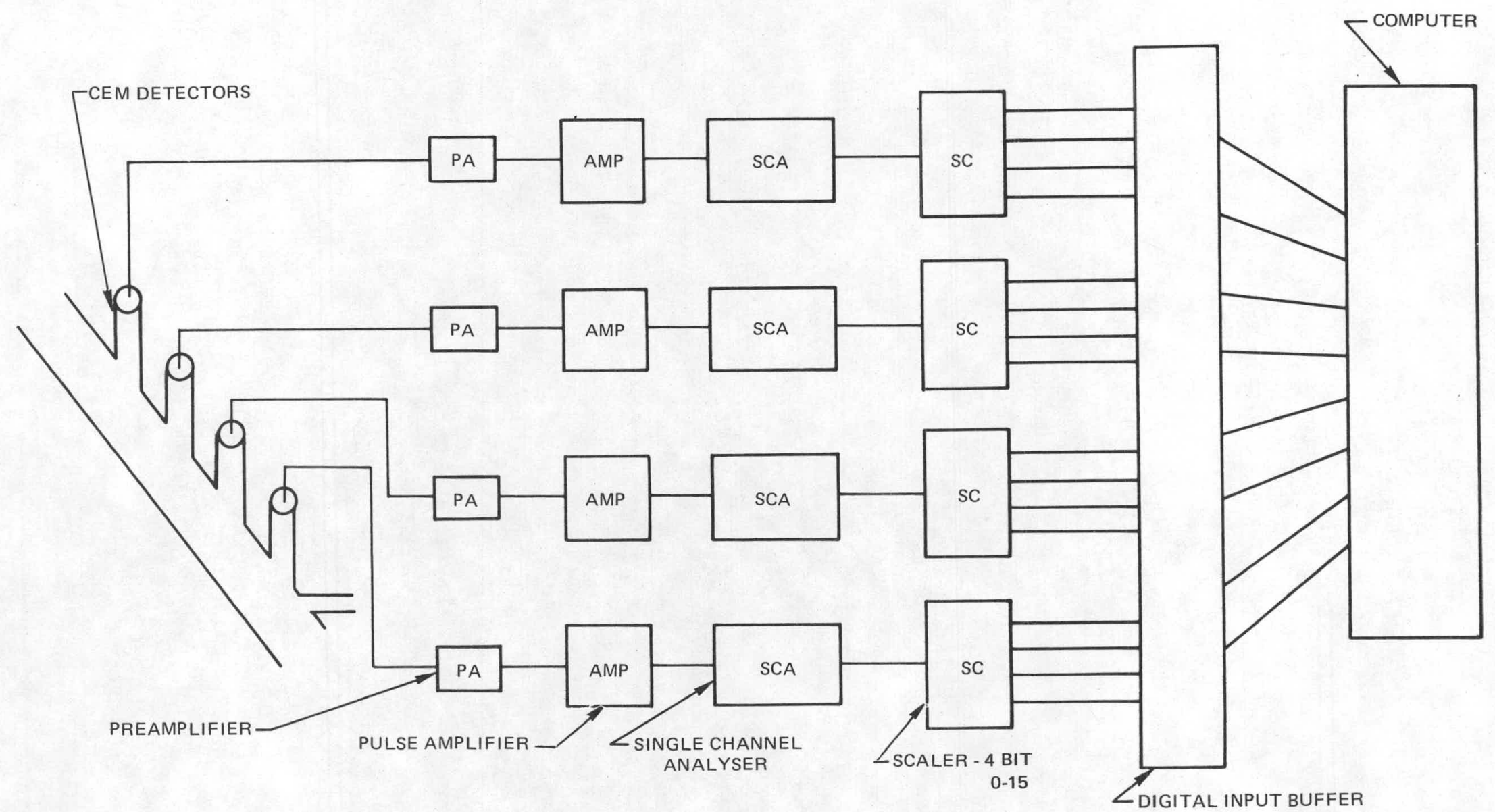




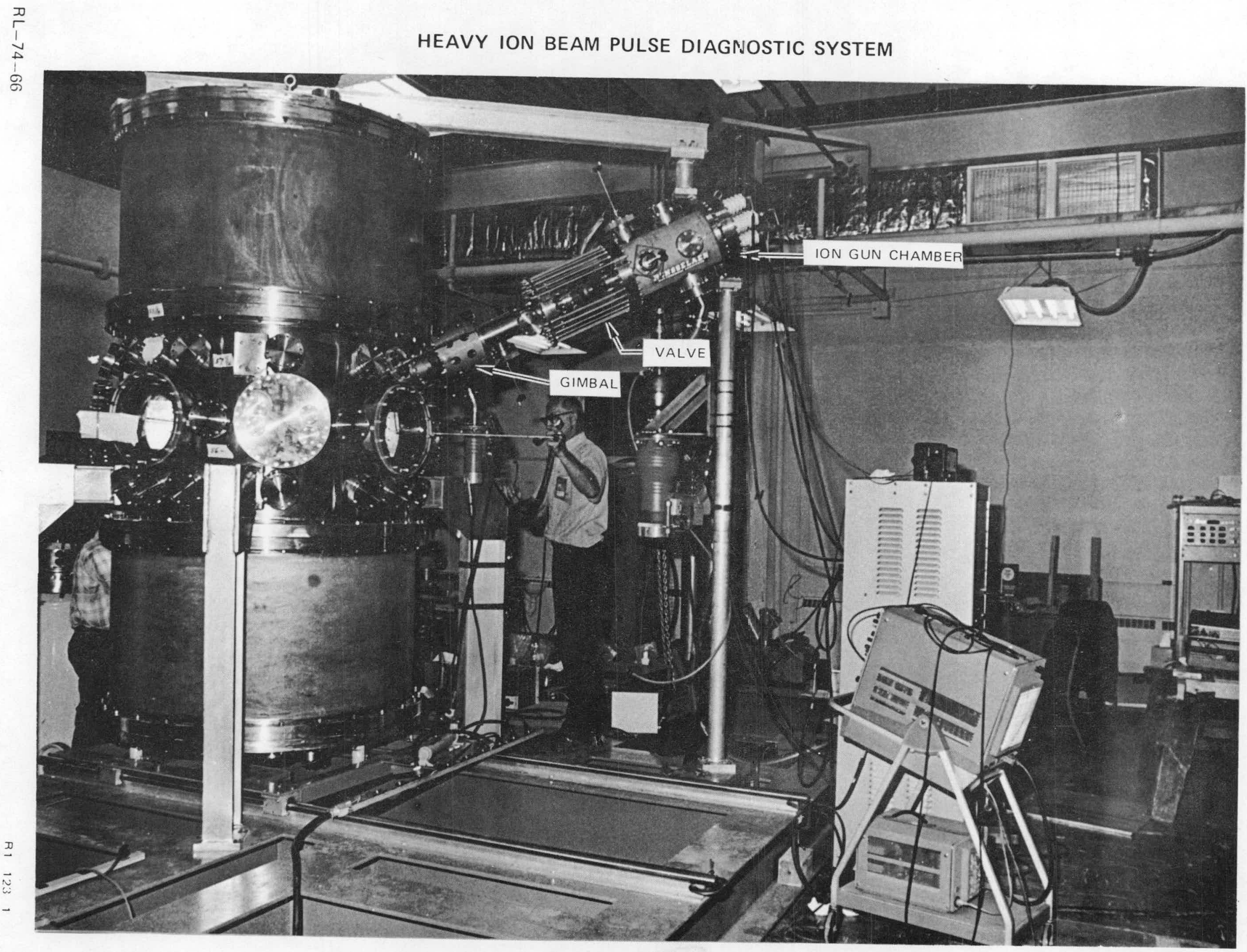


MICROWAVE INTERFEROMETER
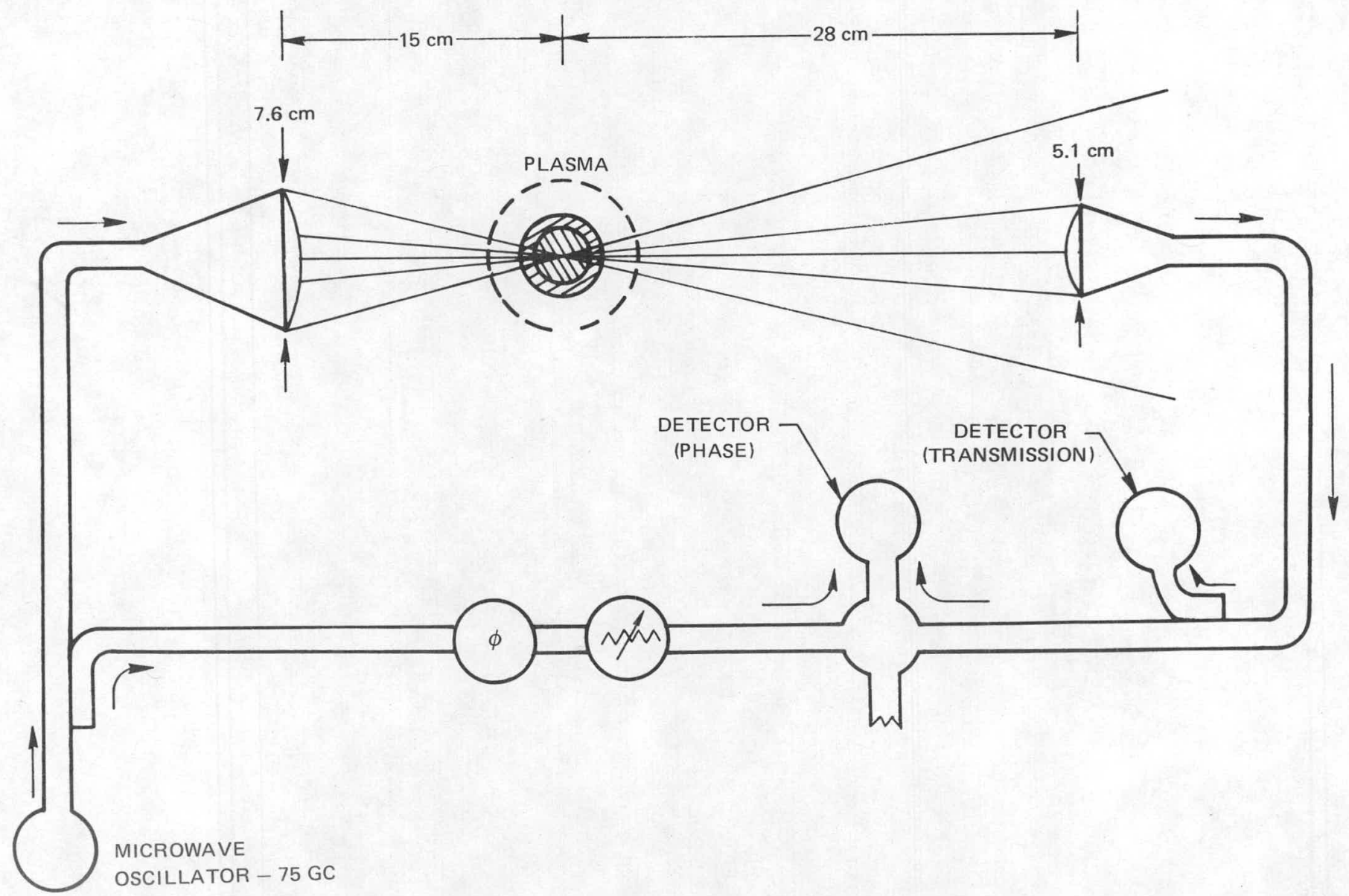


\section{COMPUTER DISPLAY OF DATA FROM CURRENT COLLECTORS}

FREE EXPANSION EXPERIMENTS

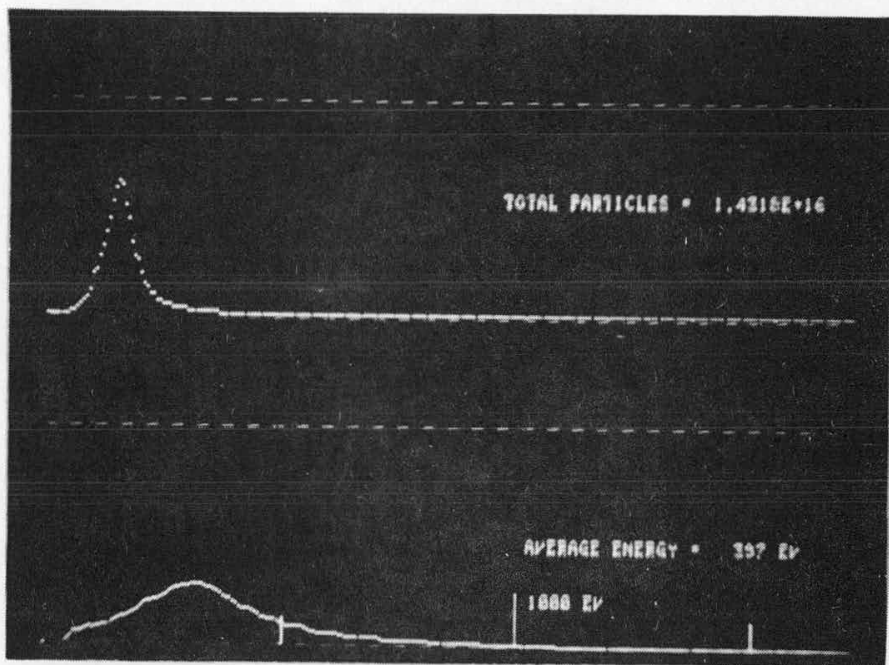

a. COLLECTORI

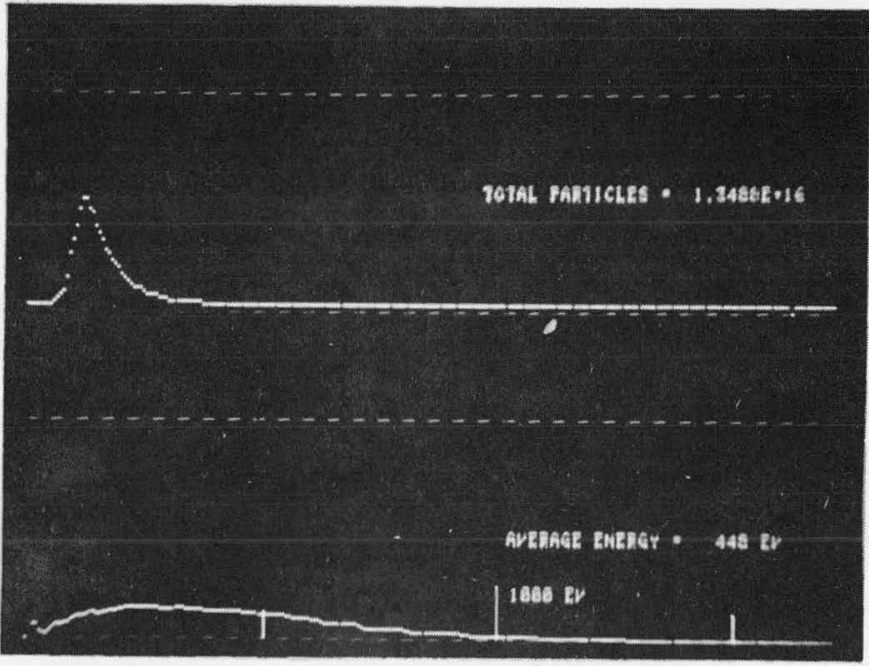

b. COLLECTOR II 
RADIAL DEPENDENCE OF THE OPTICAL EMISSION AT THE PLASMA MIDPLANE

a. SHOT 1

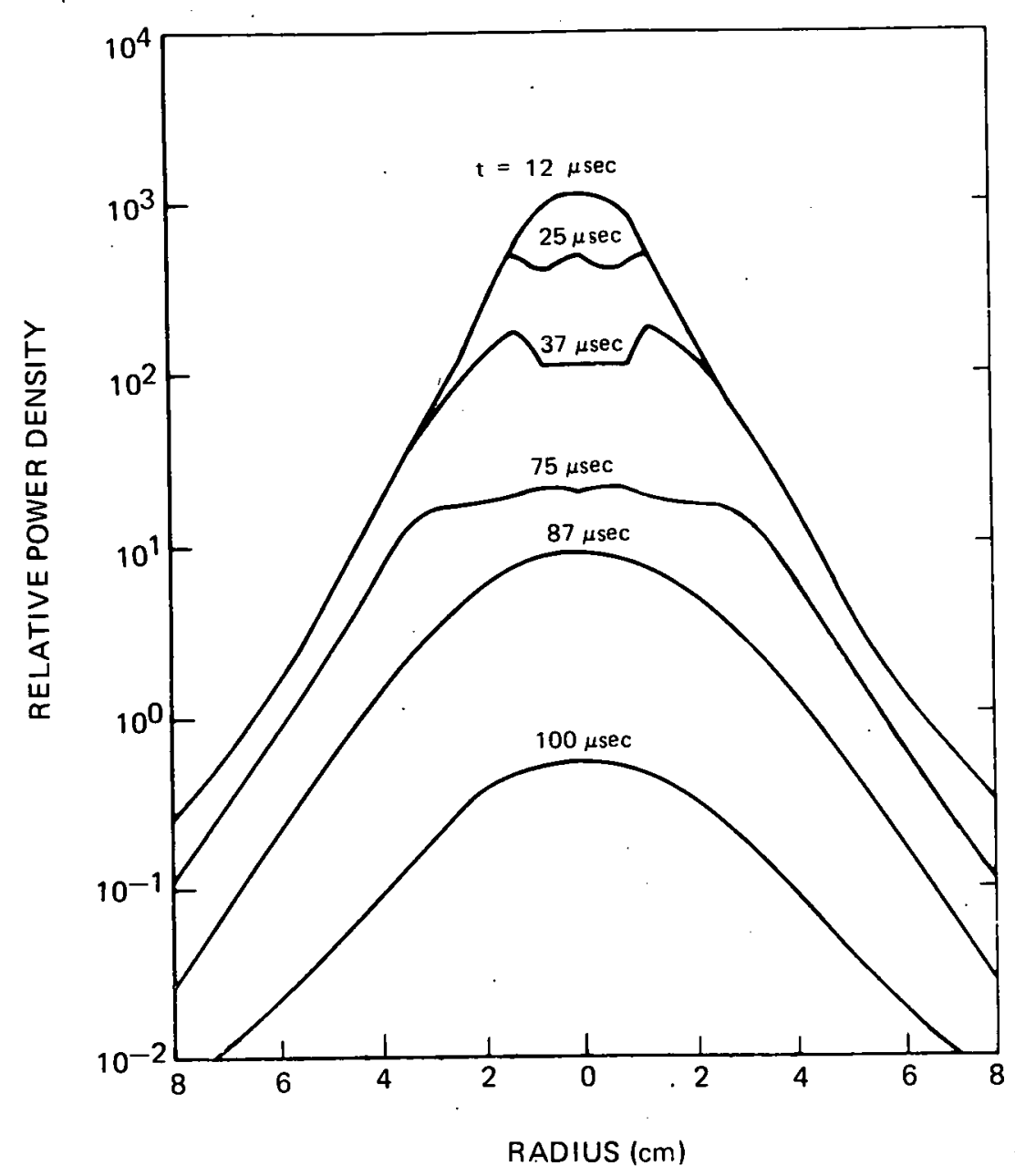

b. SHOT 2

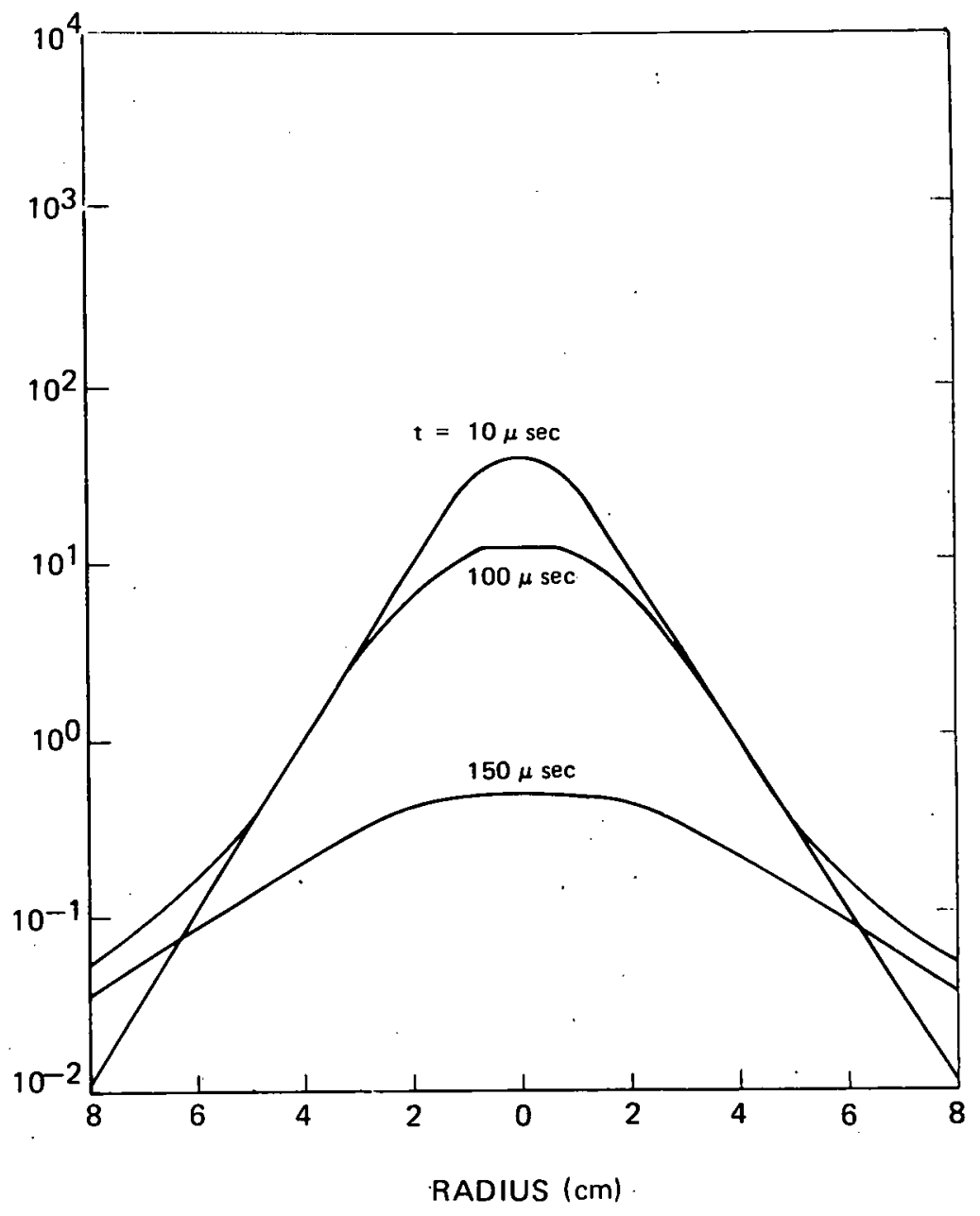


IONIZATION AND CHARGE EXCHANGE CROSS SECTIONS
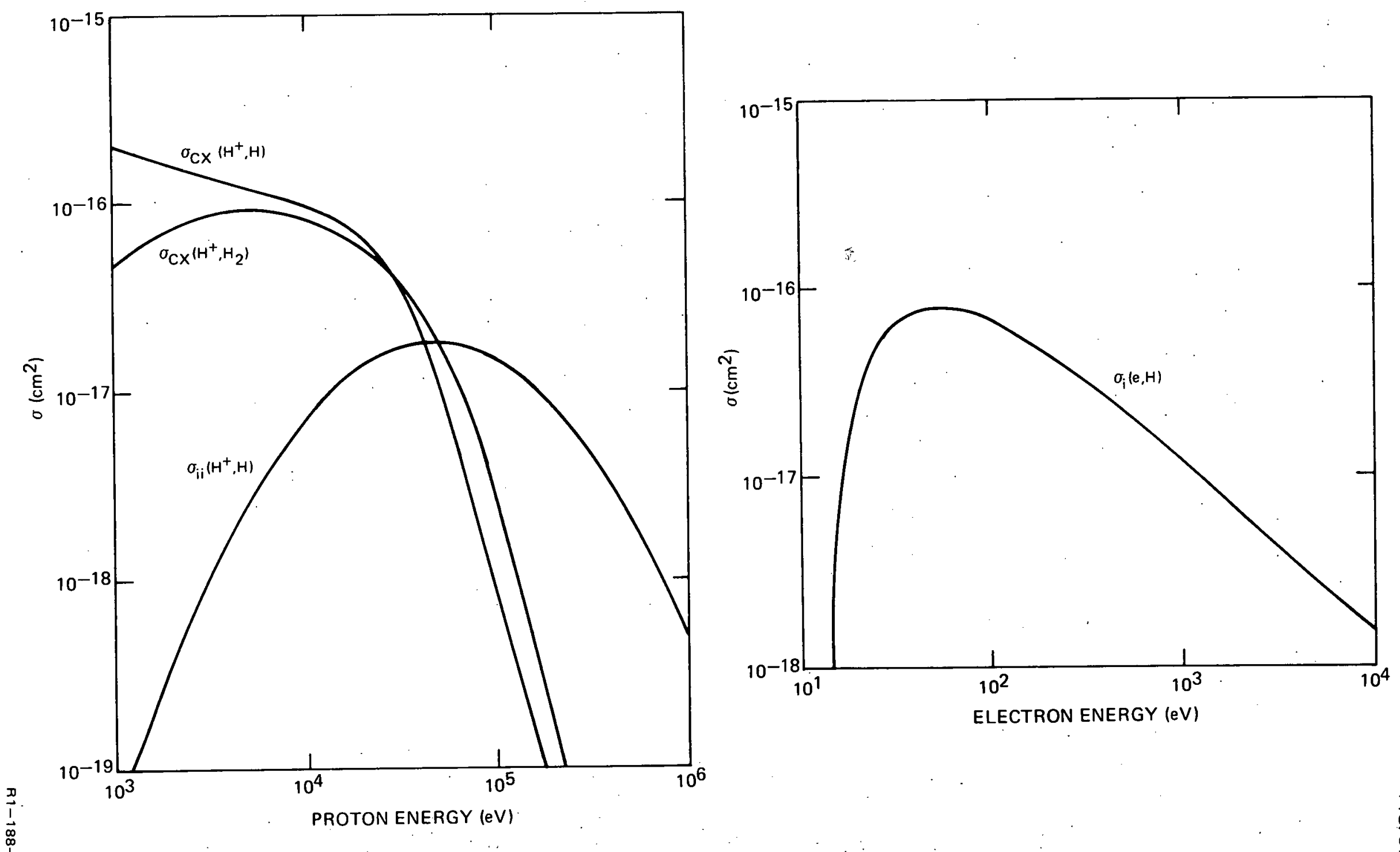

$\frac{\pi}{0}$ 


\section{LITE PLASMA DENSITIES}

$\begin{array}{lccccc}\mathrm{H}^{+} & \mathrm{e} & \mathrm{Li}^{3+} & \mathrm{H}_{\mathrm{B}}^{+} & \text {INJECTION BEAM } \\ \mathrm{n}(0)=2.5 \times 10^{13} & 10^{14} & 2.5 \times 10^{13} & 0 & \mathrm{~cm}^{-3} & 1=1.0 \mathrm{amp} \\ \mathrm{E}(0)=500 & 500 & 500 & -\mathrm{eV} & \mathrm{E}_{\mathrm{b}}=10 \mathrm{keV}\end{array}$

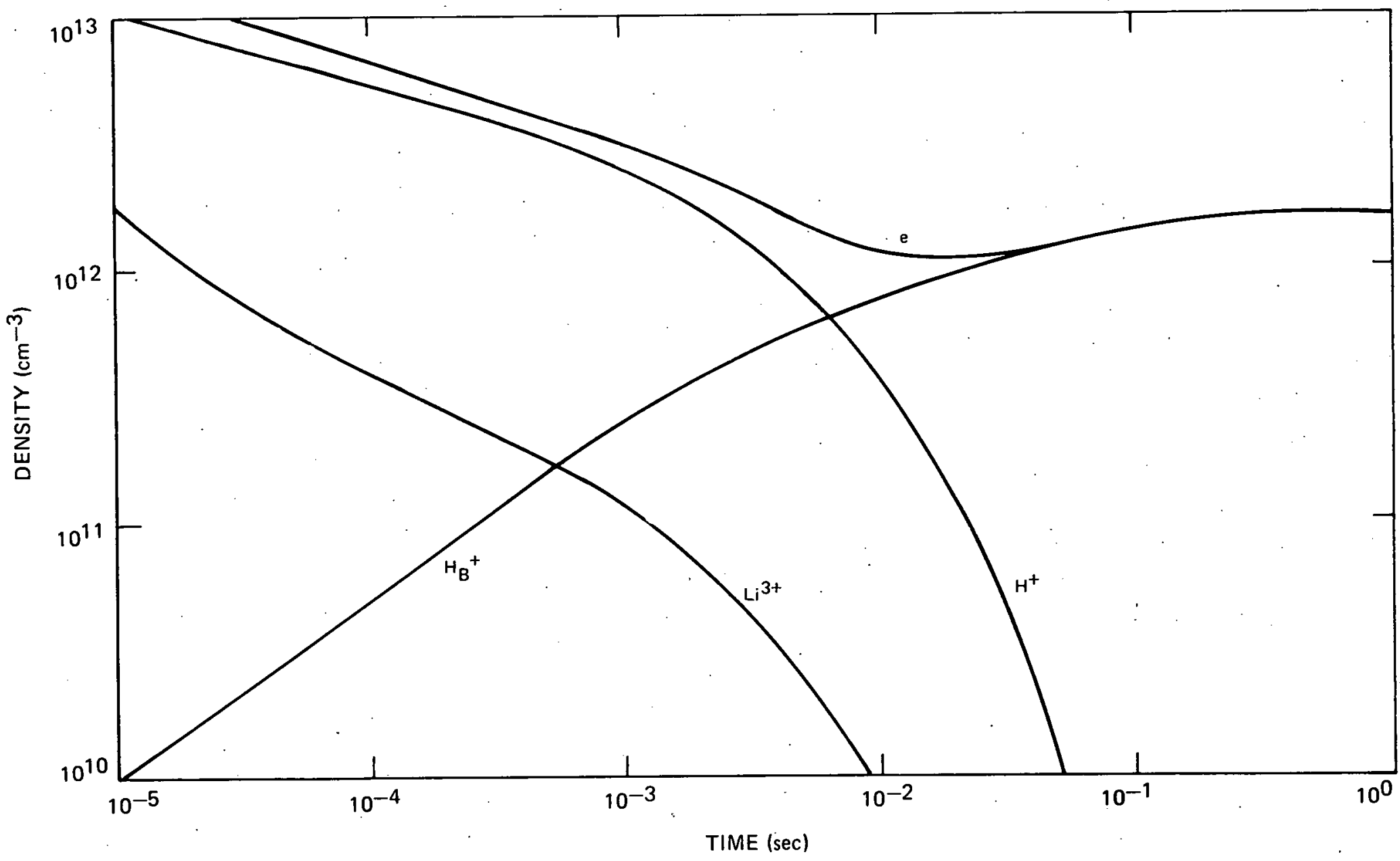




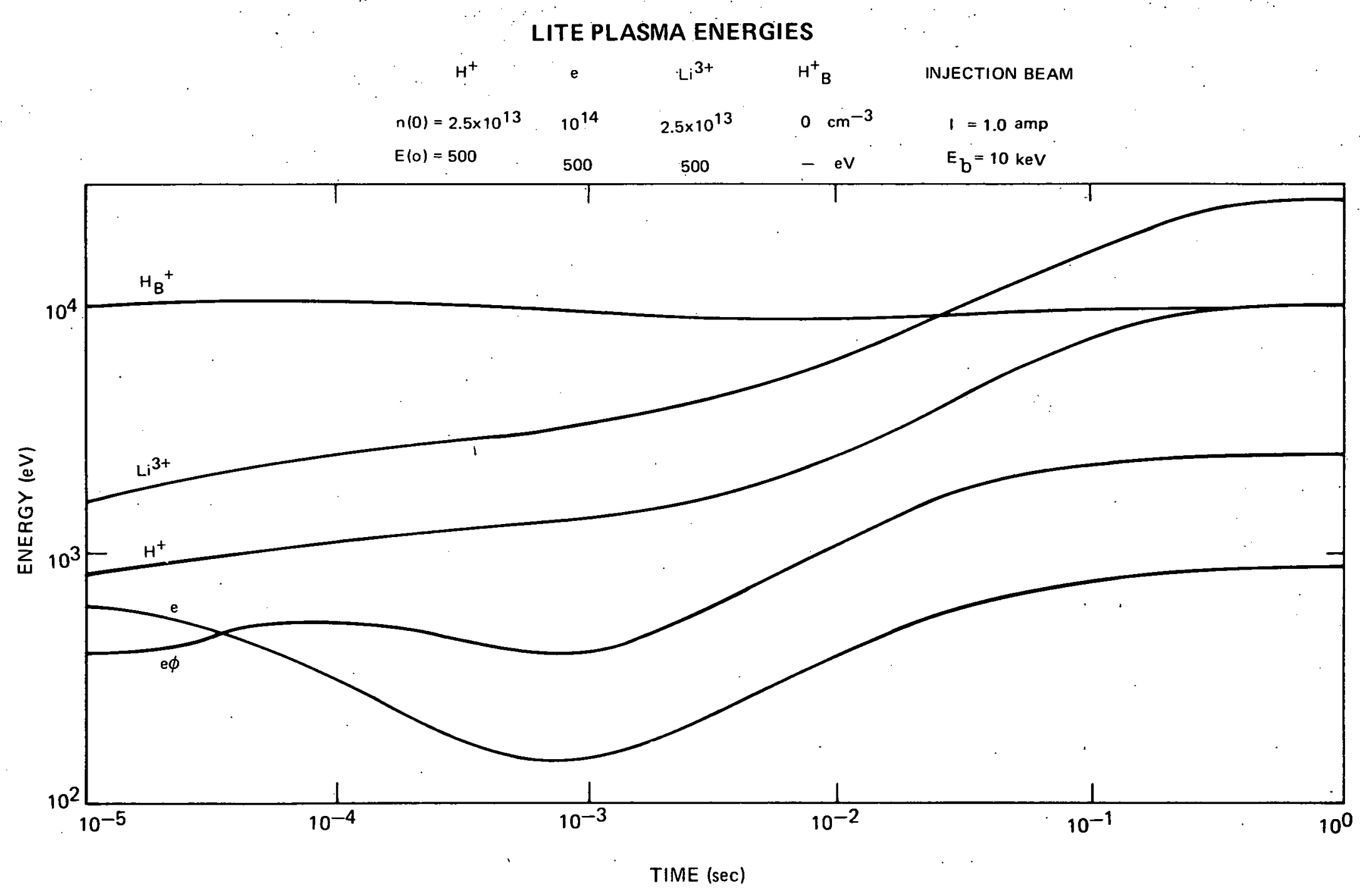


LITE PLASMA DENSITIES LID

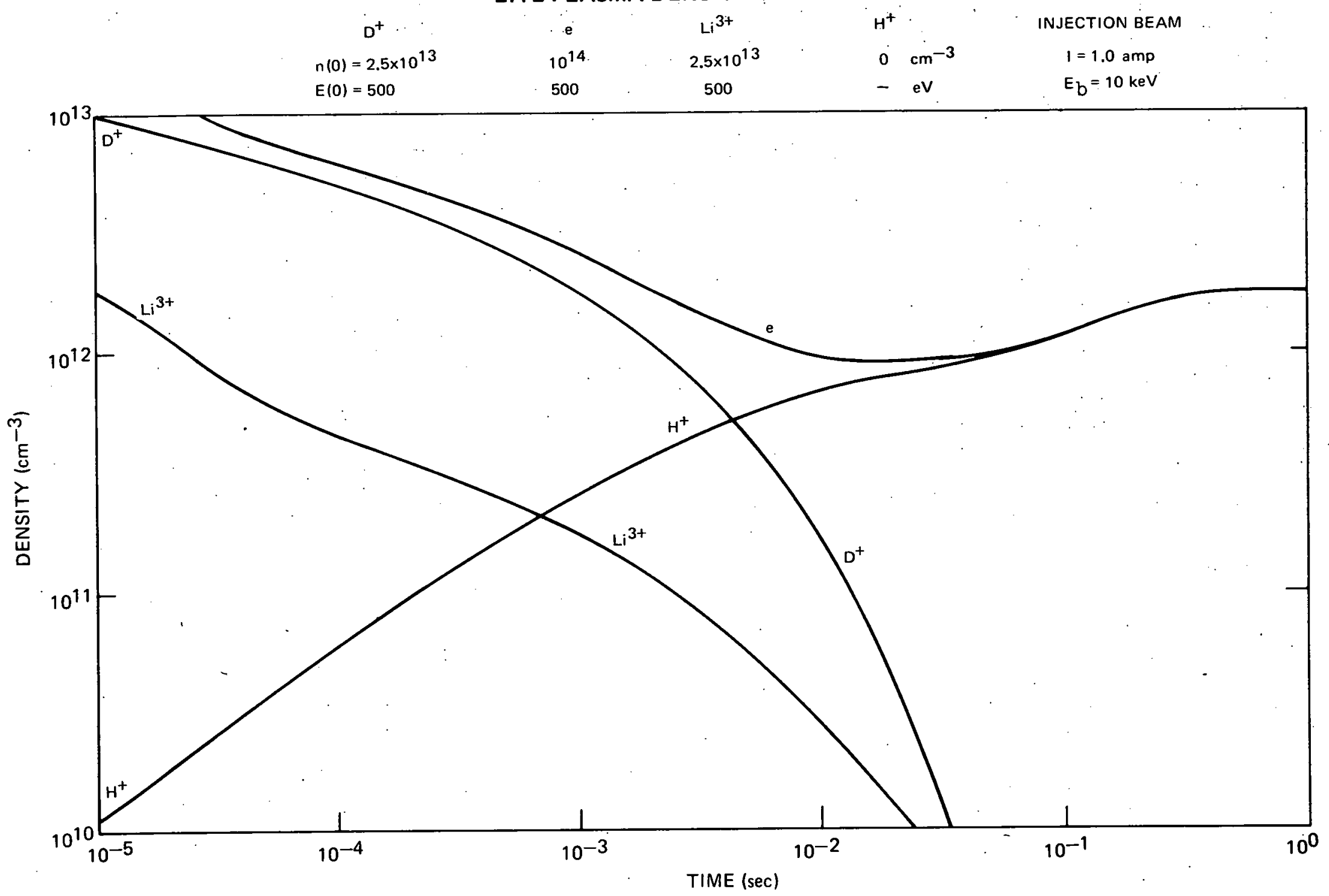




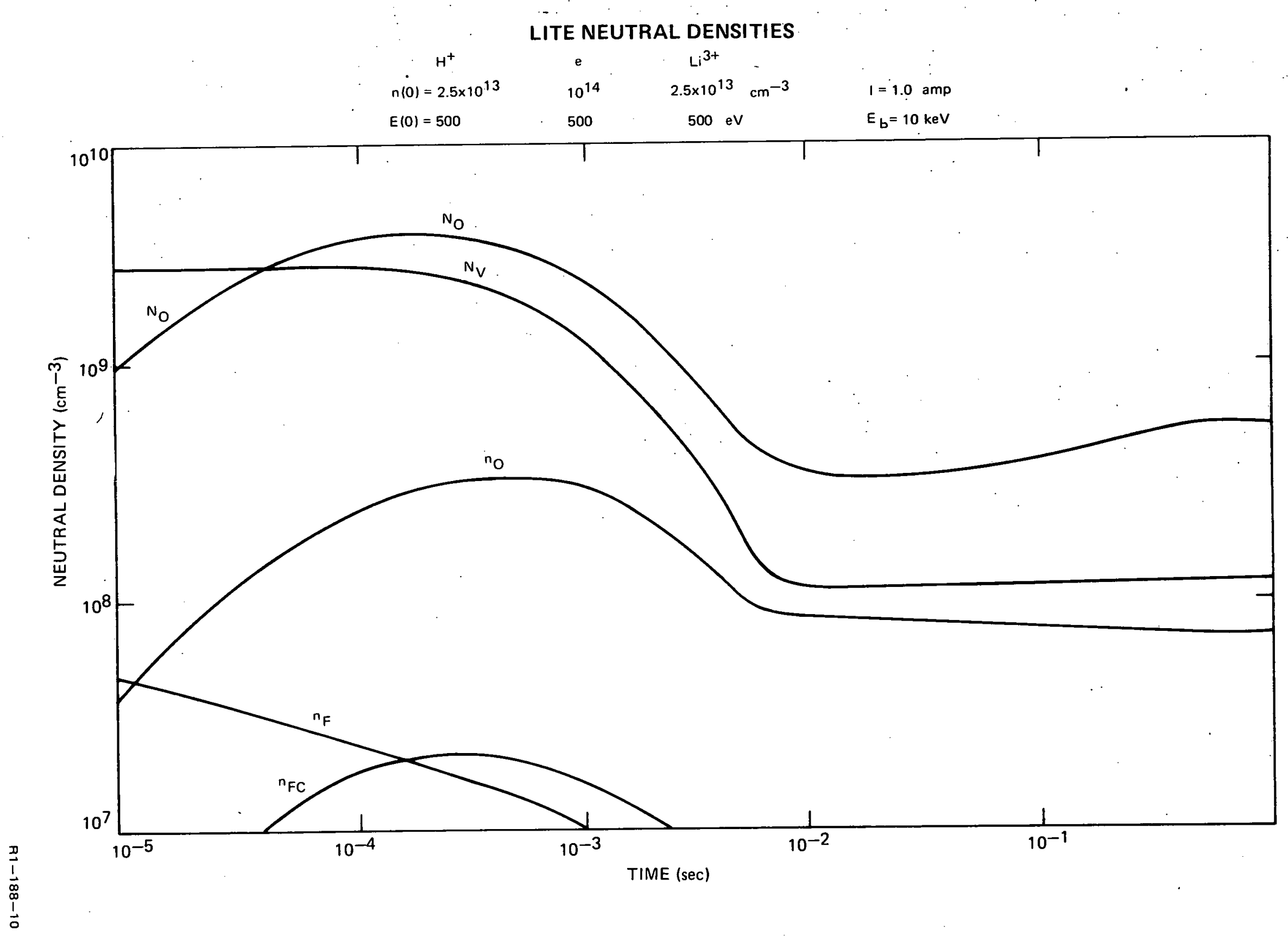




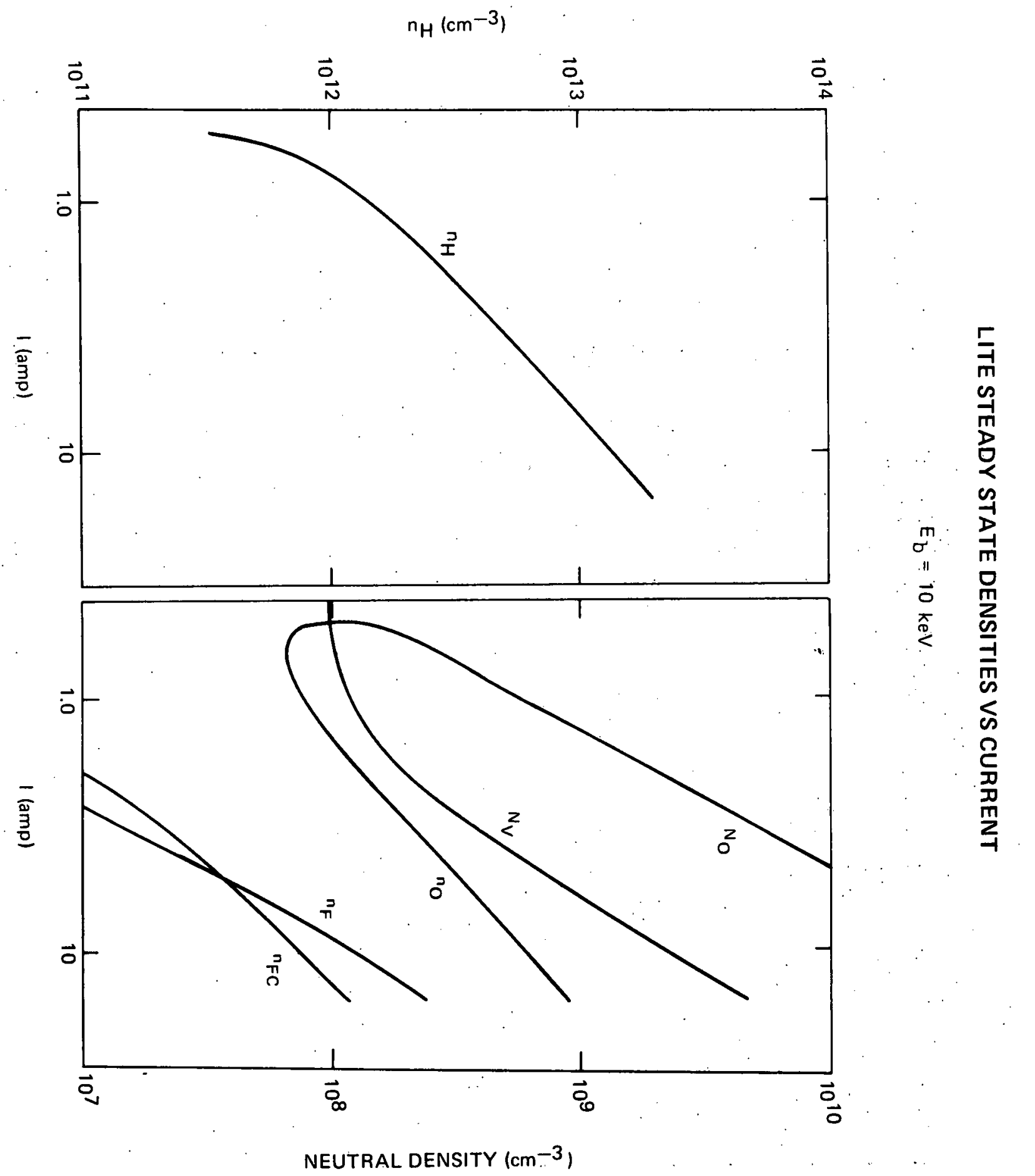




\section{LITE PLASMA DENSITIES}

$\begin{array}{lccc}\quad \mathrm{H}^{+} & \mathrm{e} & \mathrm{Li}^{3+} & \text { INJECTION BEAM } \\ n(0)=2.5 \times 10^{13} & 10^{14} & 2.5 \times 10^{13} \mathrm{~cm}^{-3} & \mathrm{I}=1.0 \mathrm{amp} \\ \mathrm{E}(0)=500 & 500 & 500 \mathrm{eV} & E_{\mathrm{b}}=10 \mathrm{keV}\end{array}$

is

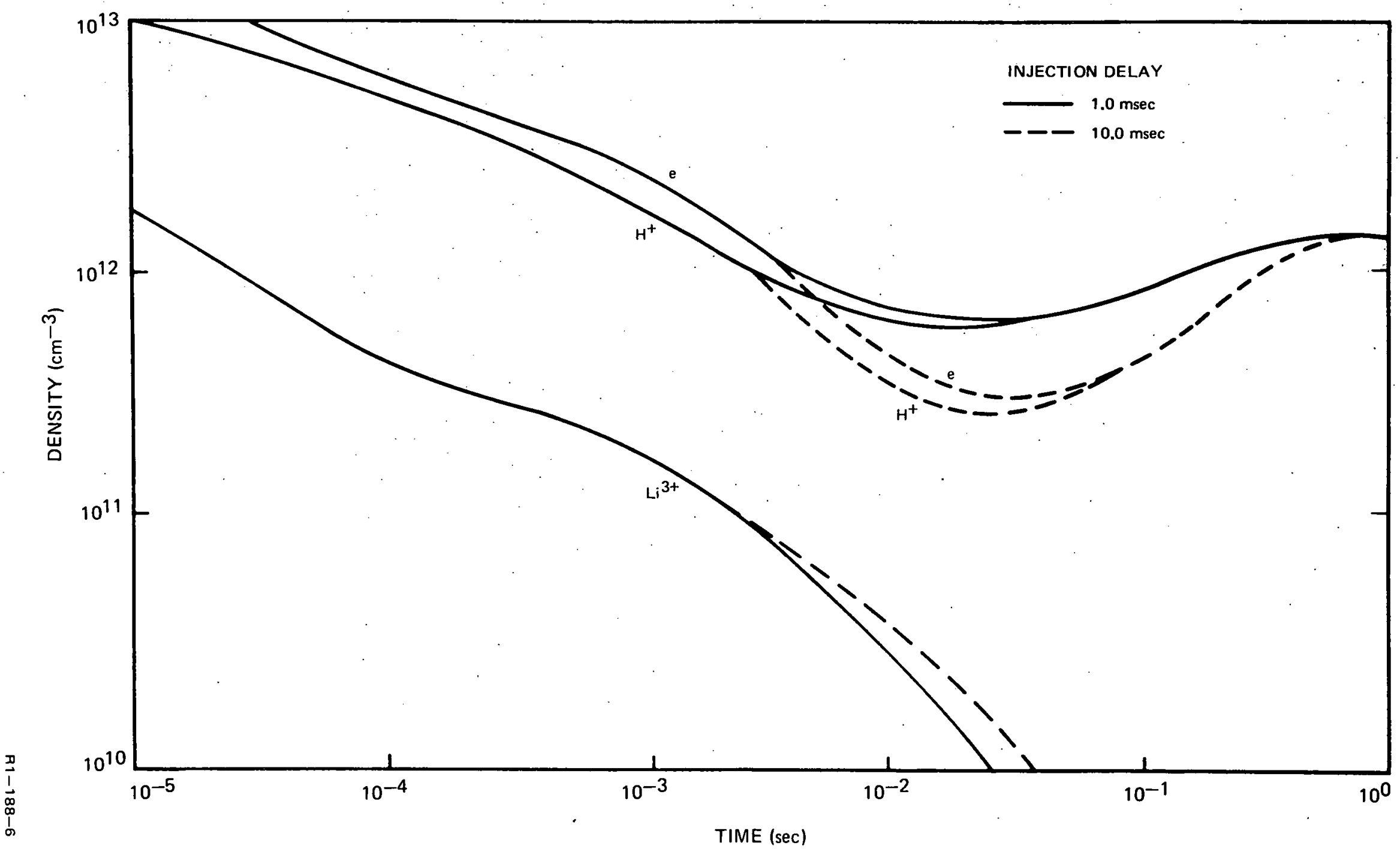




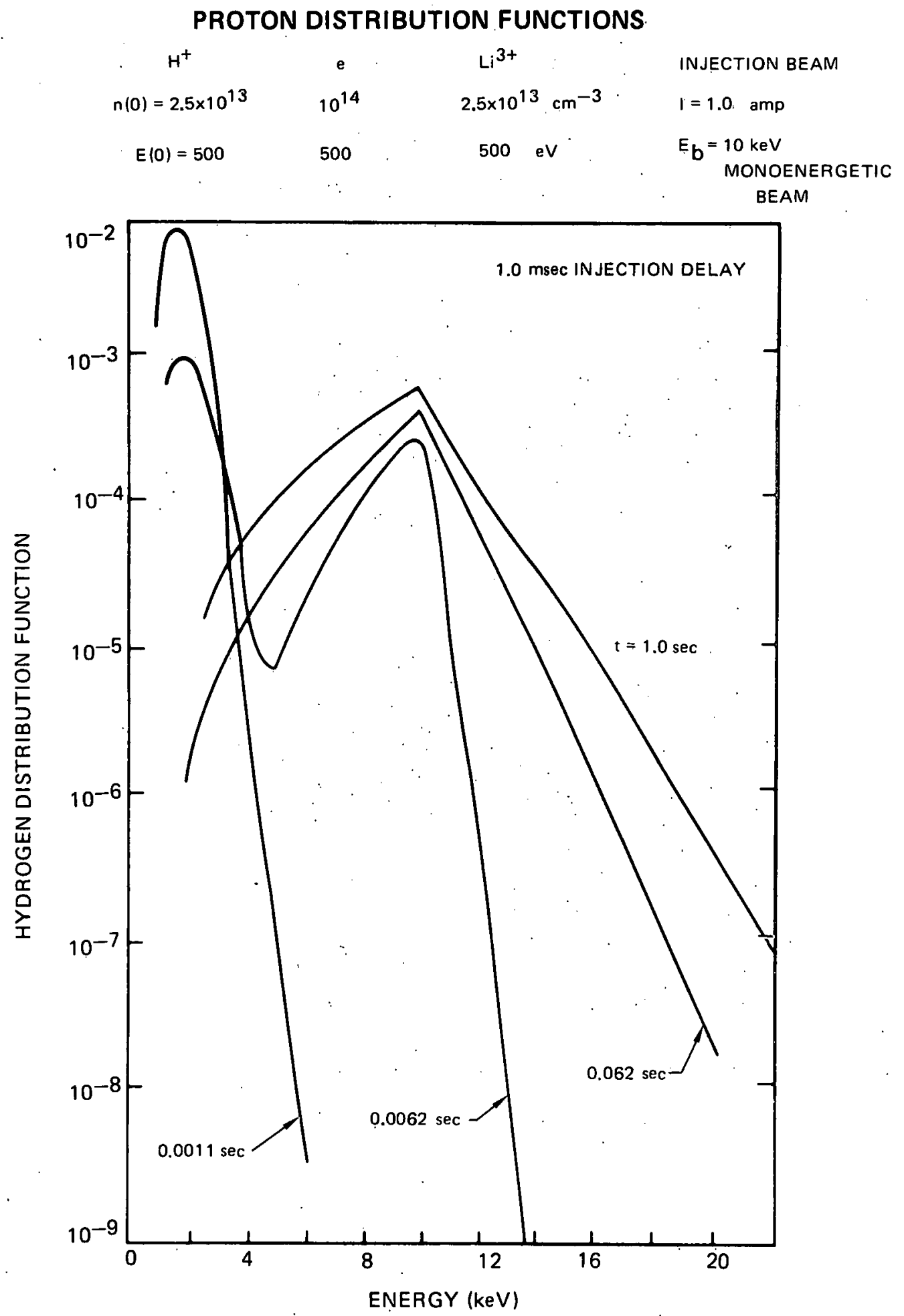


PROTON DISTRIBUTION FUNCTIONS

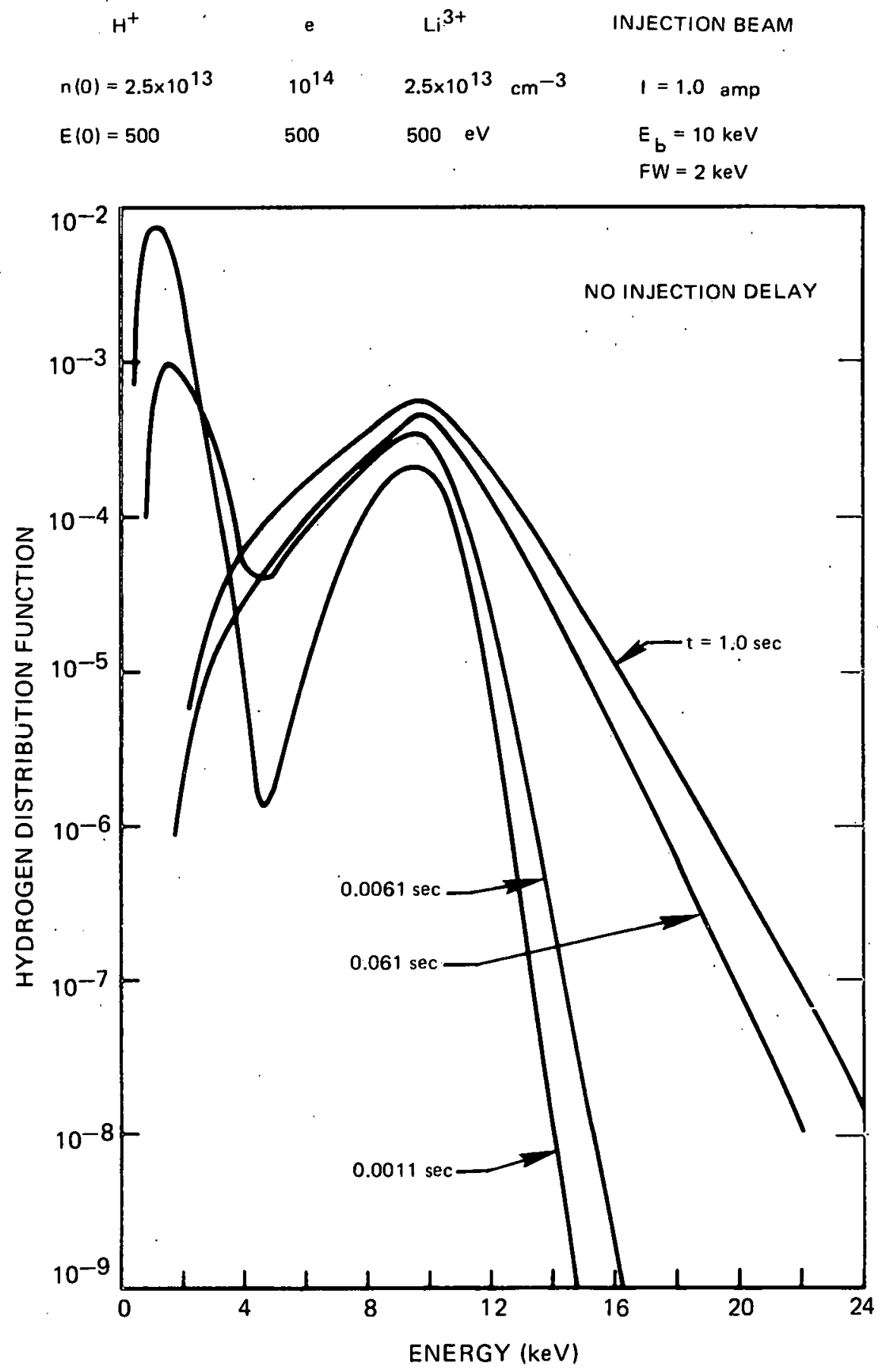




\section{PROTON DISTRIBUTION FUNCTIONS}

$\begin{array}{cccc}\mathrm{H}^{+} & \mathrm{e} & \mathrm{Li}^{3+} & \text { INJECTION BEAM } \\ \mathrm{n}(0)=2.5 \times 10^{13} & 10^{14} & 2.5 \times 10^{13} \mathrm{~cm}^{-3} & 1=1.0 \mathrm{amp} \\ \mathrm{E}(0)=500 & 500 & 500 \mathrm{eV} & \begin{array}{l}E_{\mathrm{b}}=10 \mathrm{keV} \\ \end{array} \\ & & & \mathrm{FW}=10 \mathrm{keV}\end{array}$

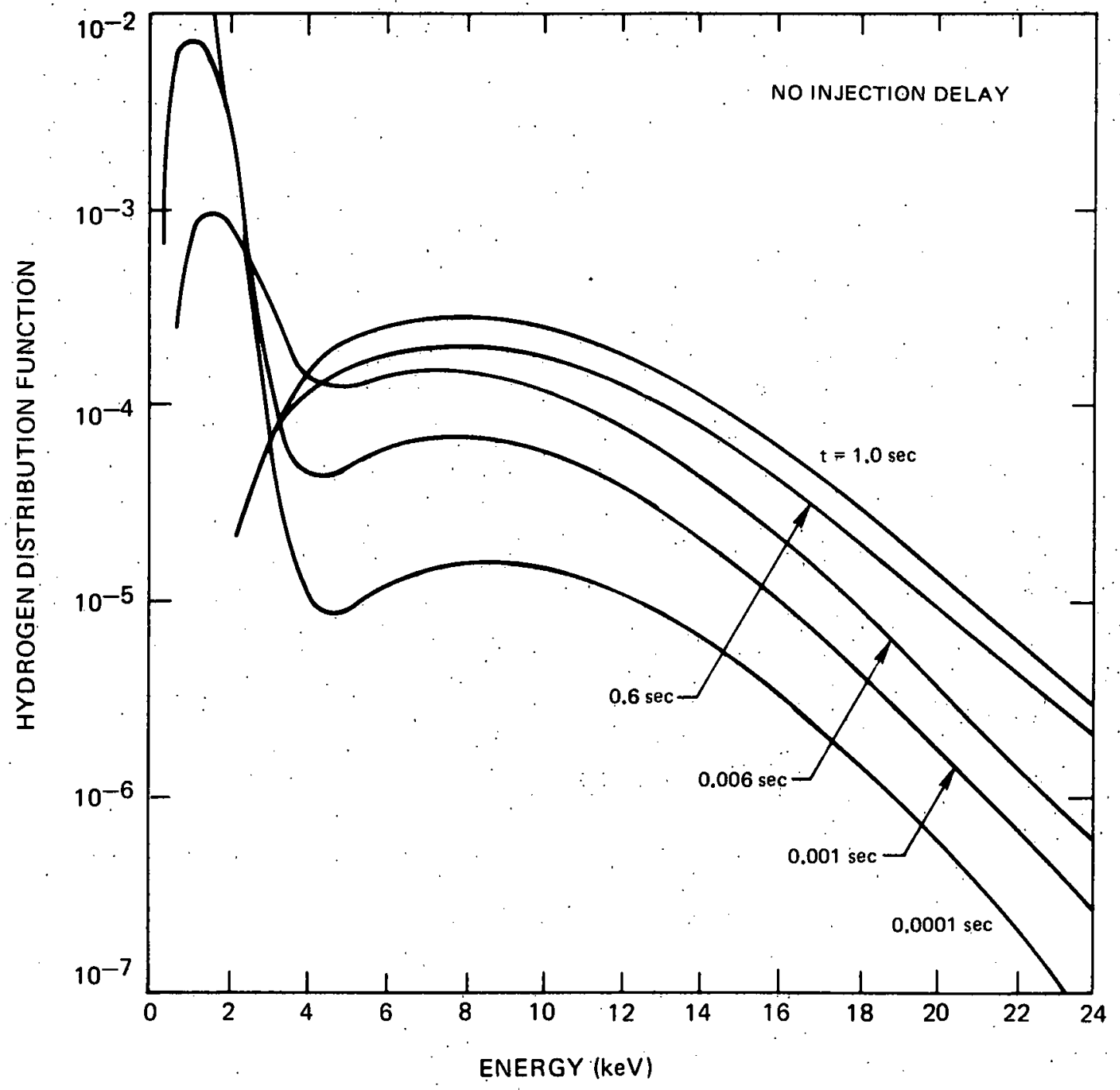




\section{LITE PLASMA DENSITIES}

INITIAL CONDITIONS: $\beta=1, B=10^{14} \mathrm{G}, \mathrm{R}(0)=1 \mathrm{~cm} \quad(54 \mu, 2.5 \mathrm{~J})$

$\begin{array}{cccc}H^{+} & \mathrm{e} & \mathrm{Li}^{3+} & \text { INJECTION BEAM } \\ n(0)=1.26 \times 10^{15} & 5.05 \times 10^{15} & 1.26 \times 10^{15} \mathrm{~cm}^{-3} & I=1.0 \mathrm{amp} \\ \mathrm{E}(0)=368.2 & 0.200 & 2583 \mathrm{eV} & E_{\mathrm{b}}=10 \mathrm{keV}\end{array}$
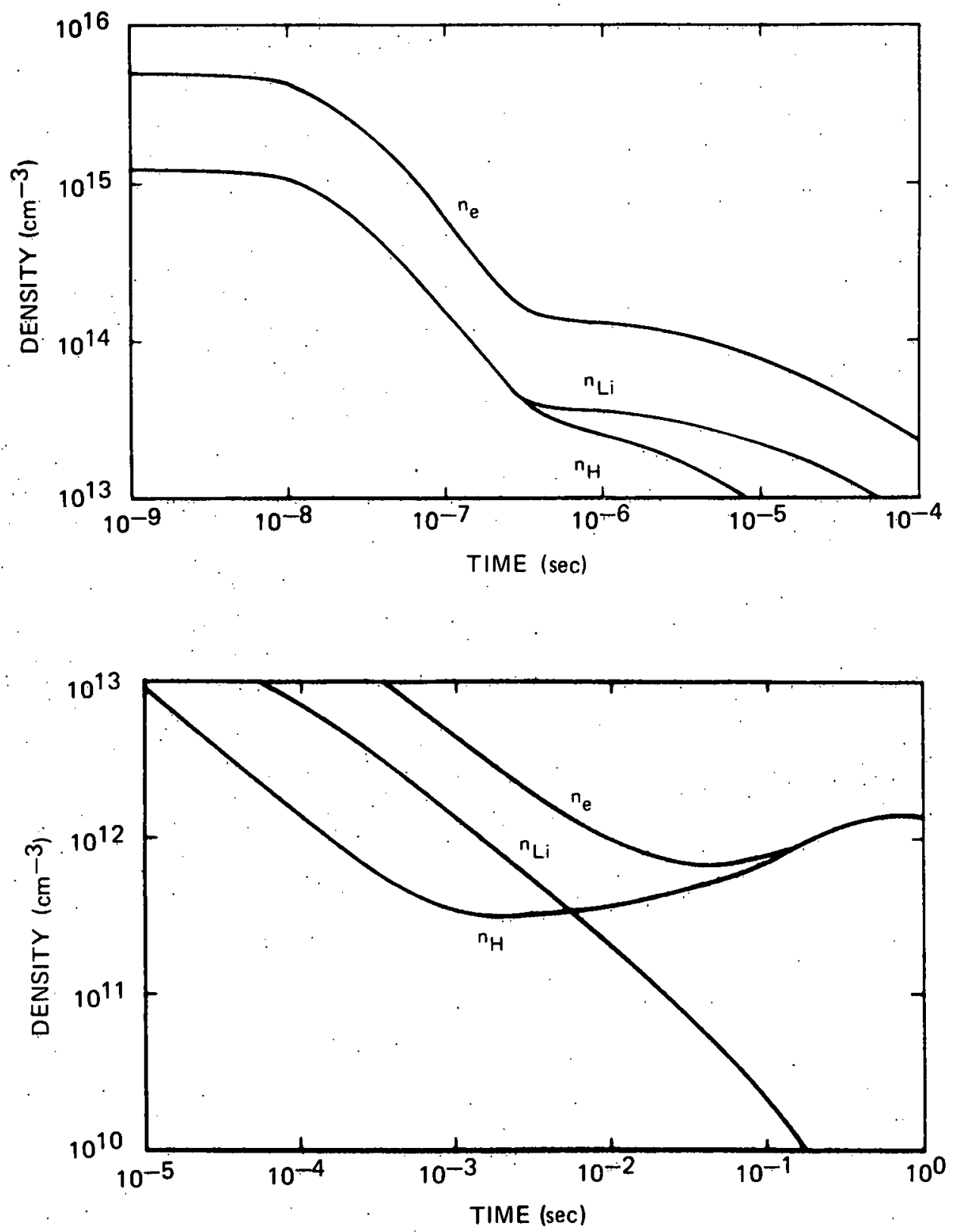


\section{LITE PLASMA ENERGIES}

INITIAL CONDITIONS: $\beta=1, B=10^{4} \mathrm{G}, \mathrm{R}(0)=1 \mathrm{~cm}(54 \mu, 2.5 \mathrm{~J})$
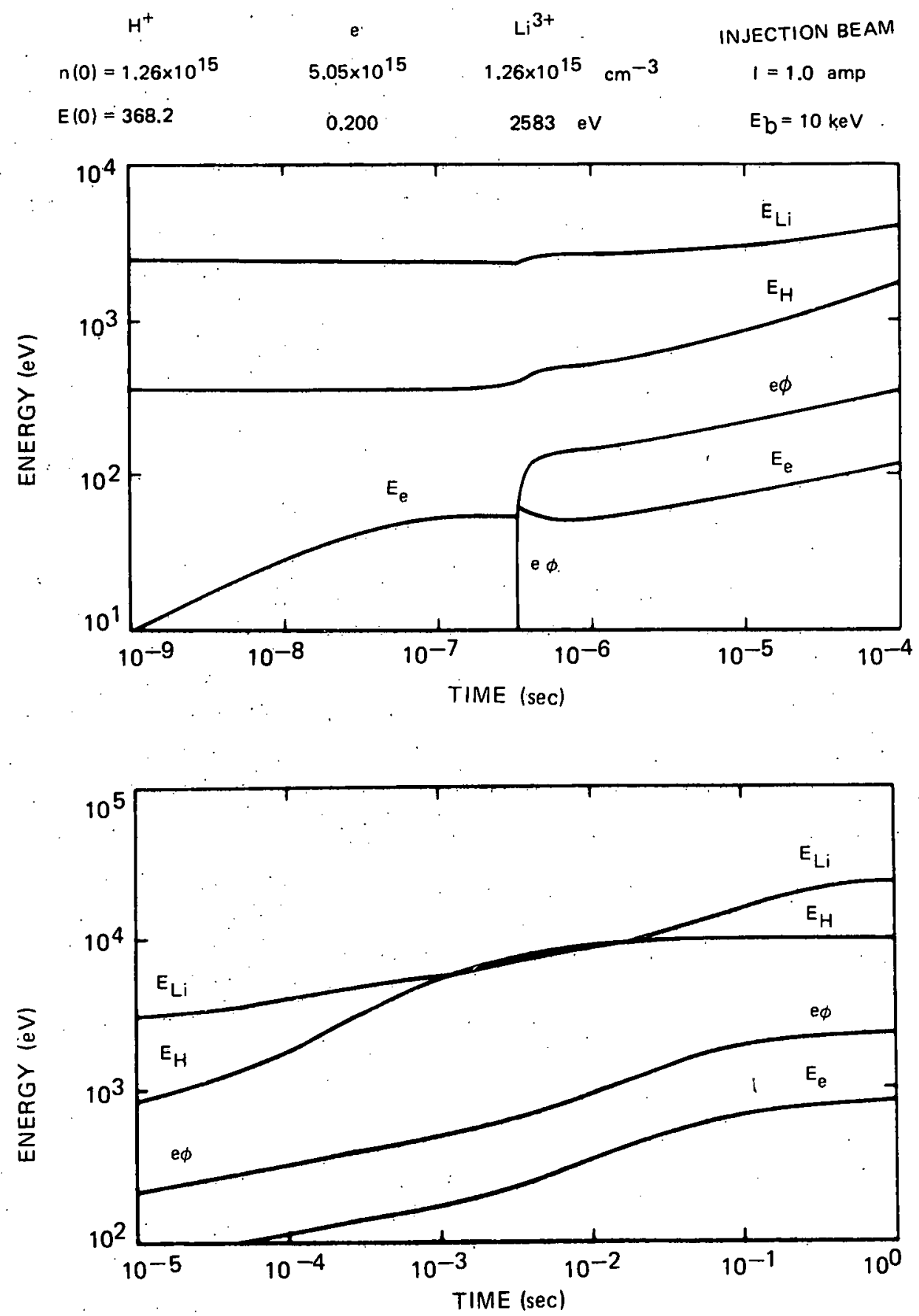
COMPUTATIONAL MODEL FOR NEUTRAL INJECTION INTO TARGET PLASMA

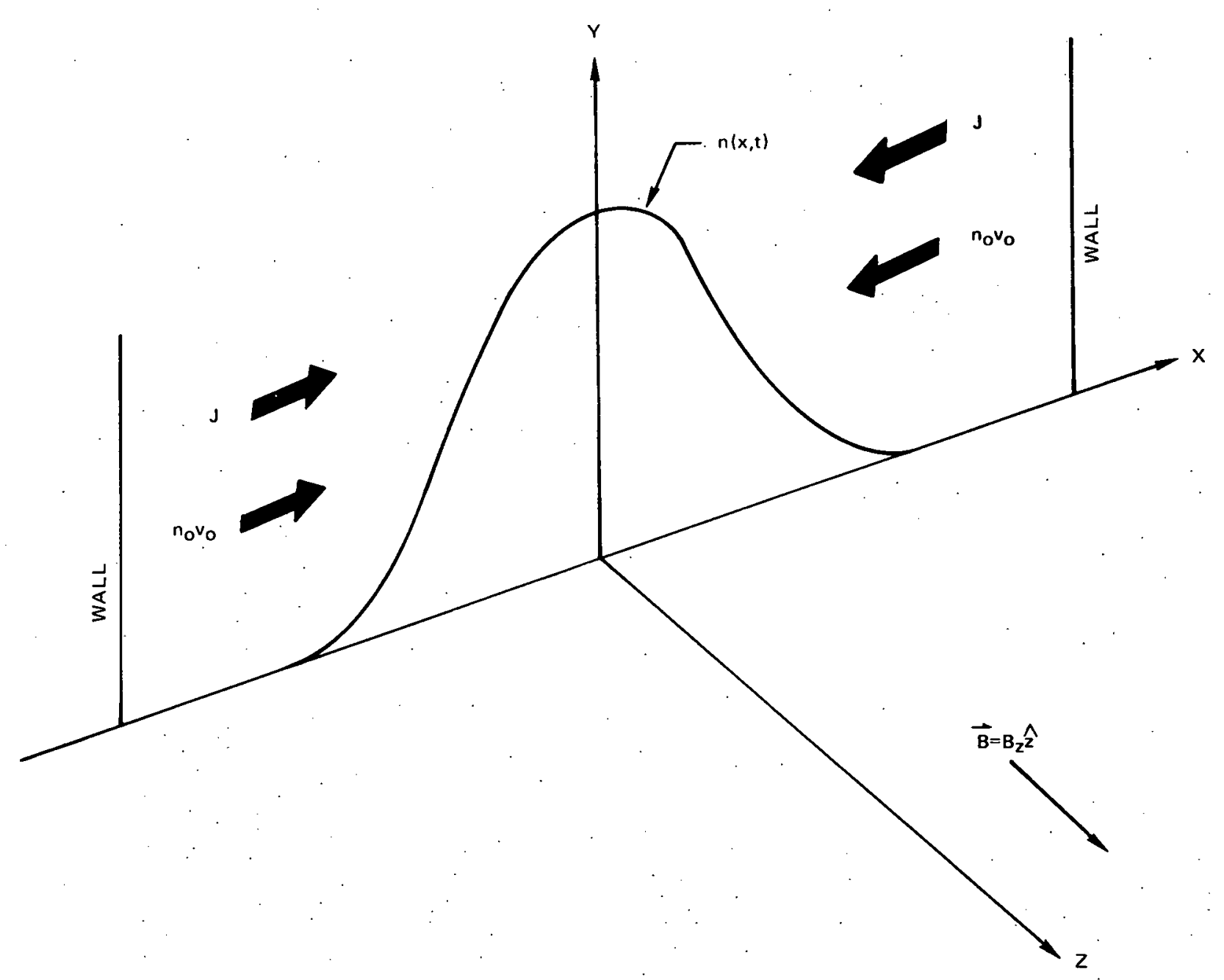


$R \mid 953100-2 \cdot 2$

FIG. 44

MECHANISM FOR CHARGE EXCHANGE DIFFUSION PROCESS

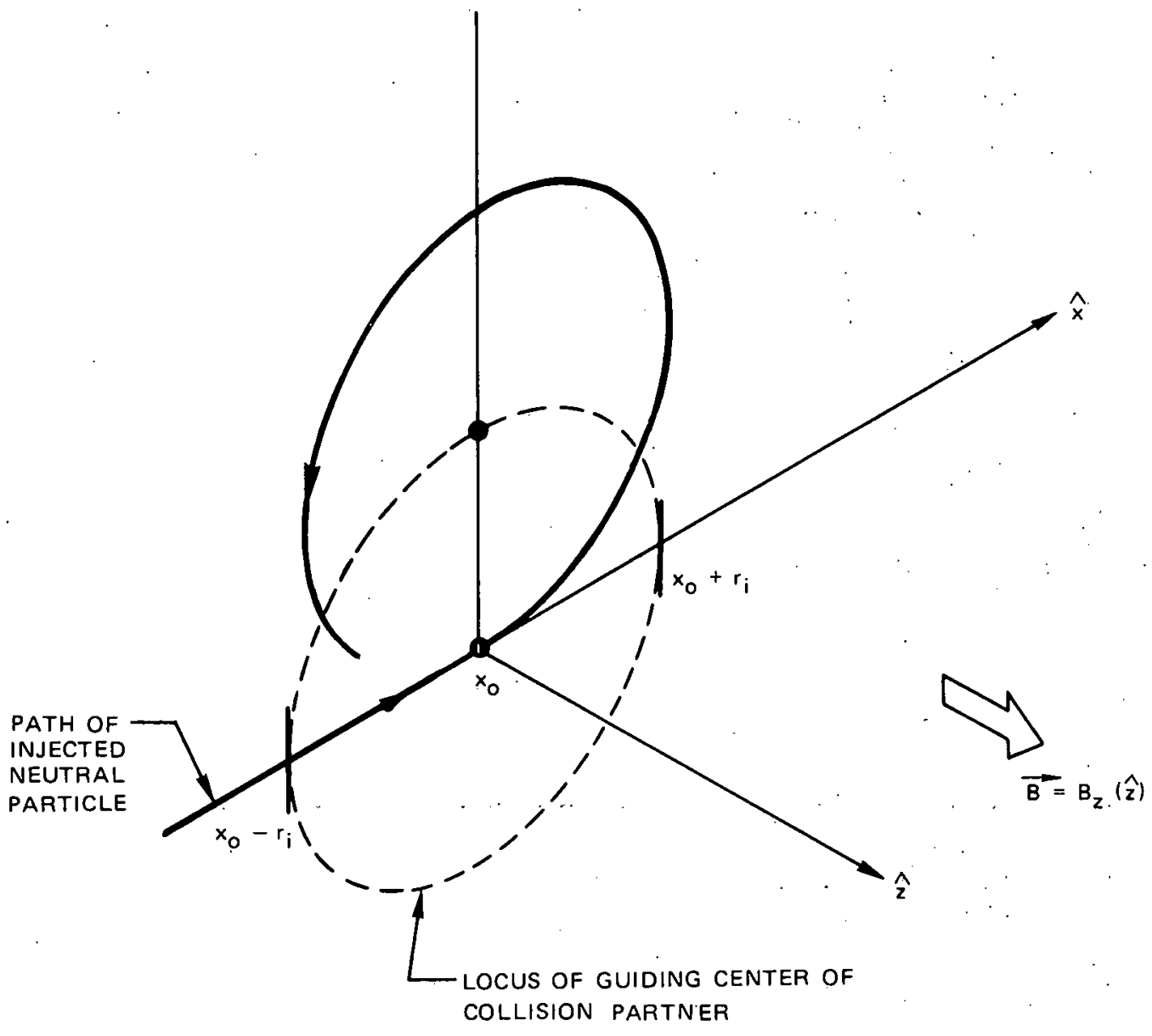

R1-152-1 


\section{SIMULATION OF BASIC PROCESSES IN PLASMA DECAY AND INJECTION SUSTENANCE}

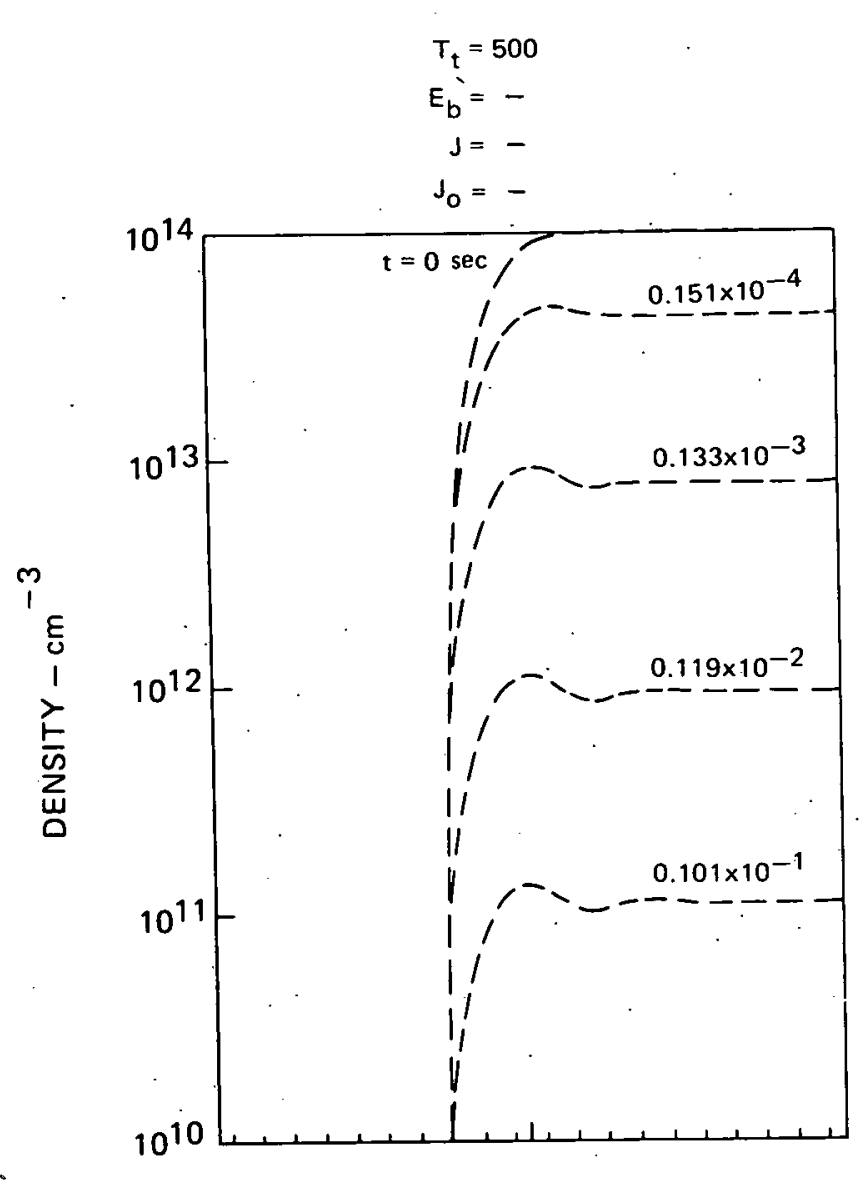

COMPUTATIONAL GRID POSITION

a. TARGET PLASMA DECAY IN PERFECT VACUUM
500

$-$

$10^{16}$

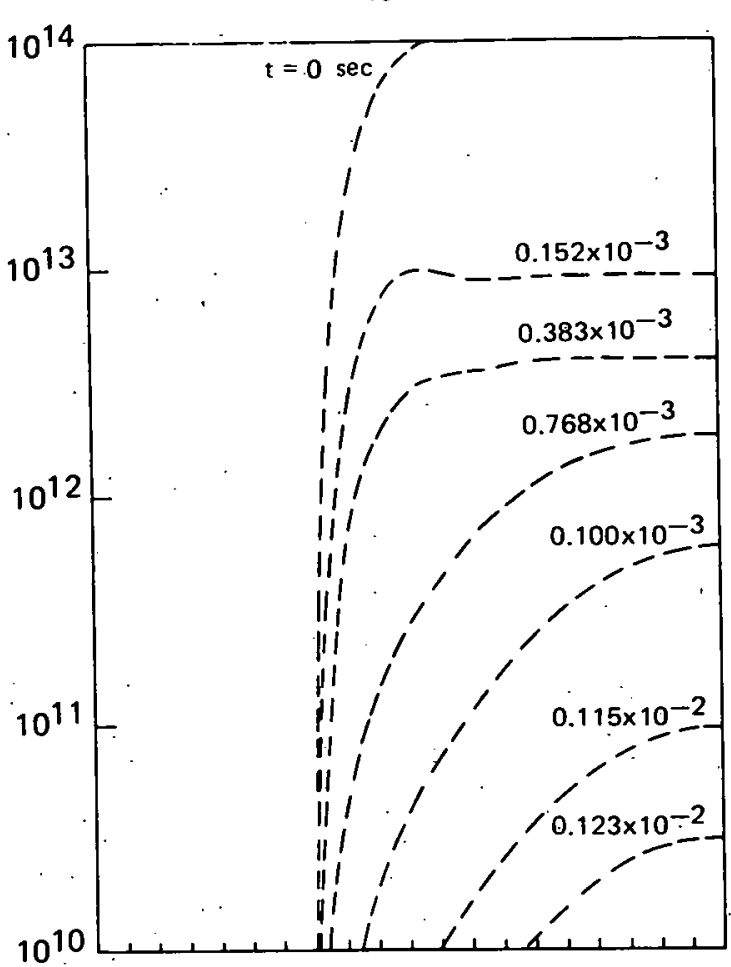

COMPUTATIONAL GRID POSITION

b. TARGET PLASMA DECAY IN BACKGROUND GAS
$500 \mathrm{eV}$

$10 \mathrm{keV}$

$16 \mathrm{~mA} / \mathrm{cm}^{2}$

$10^{16} \# / \mathrm{cm}^{2}-\mathrm{sec}$

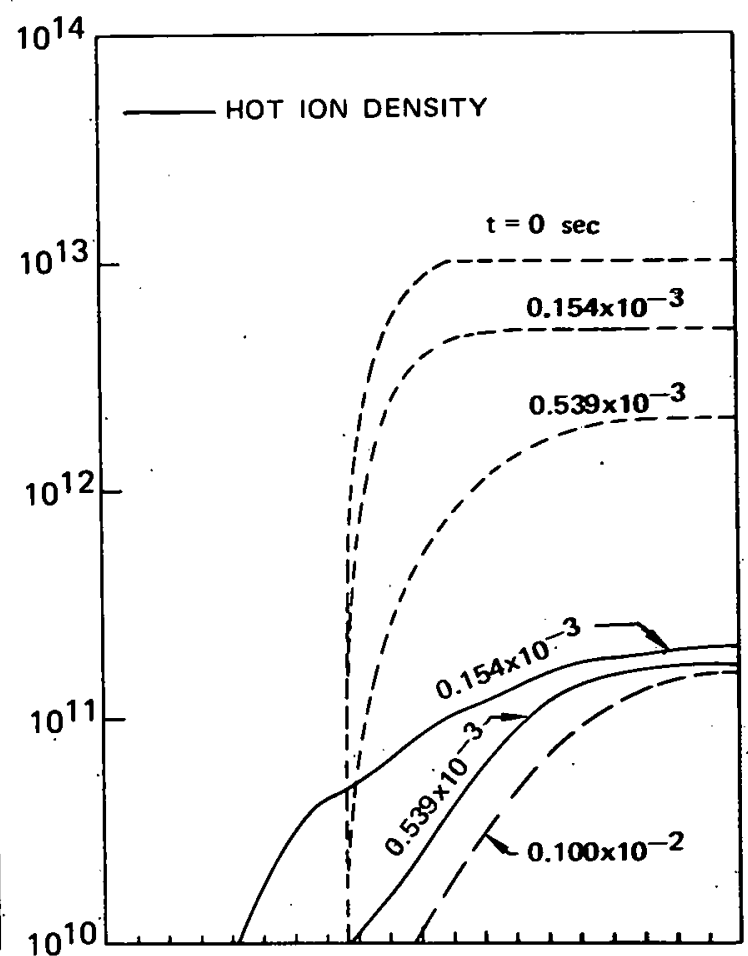

COMPUTATIONAL GRID POSITION

c. INJECTION INTO TARGET PLASMA IN BACKGROUND GAS 


\section{SUSTENANCE IN PERFECT VACUUM}

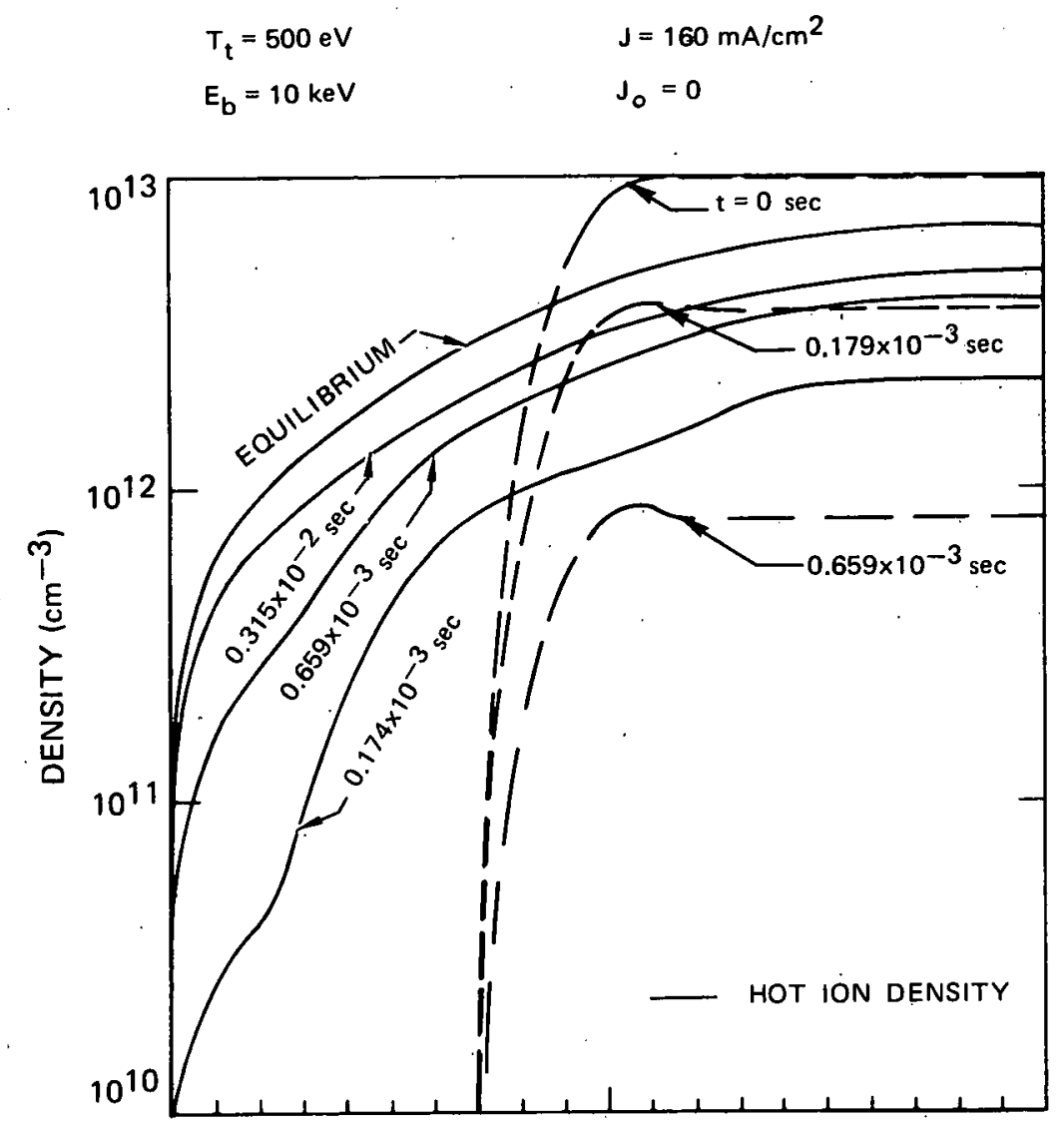

COMPUTATIONAL GRID POSITION

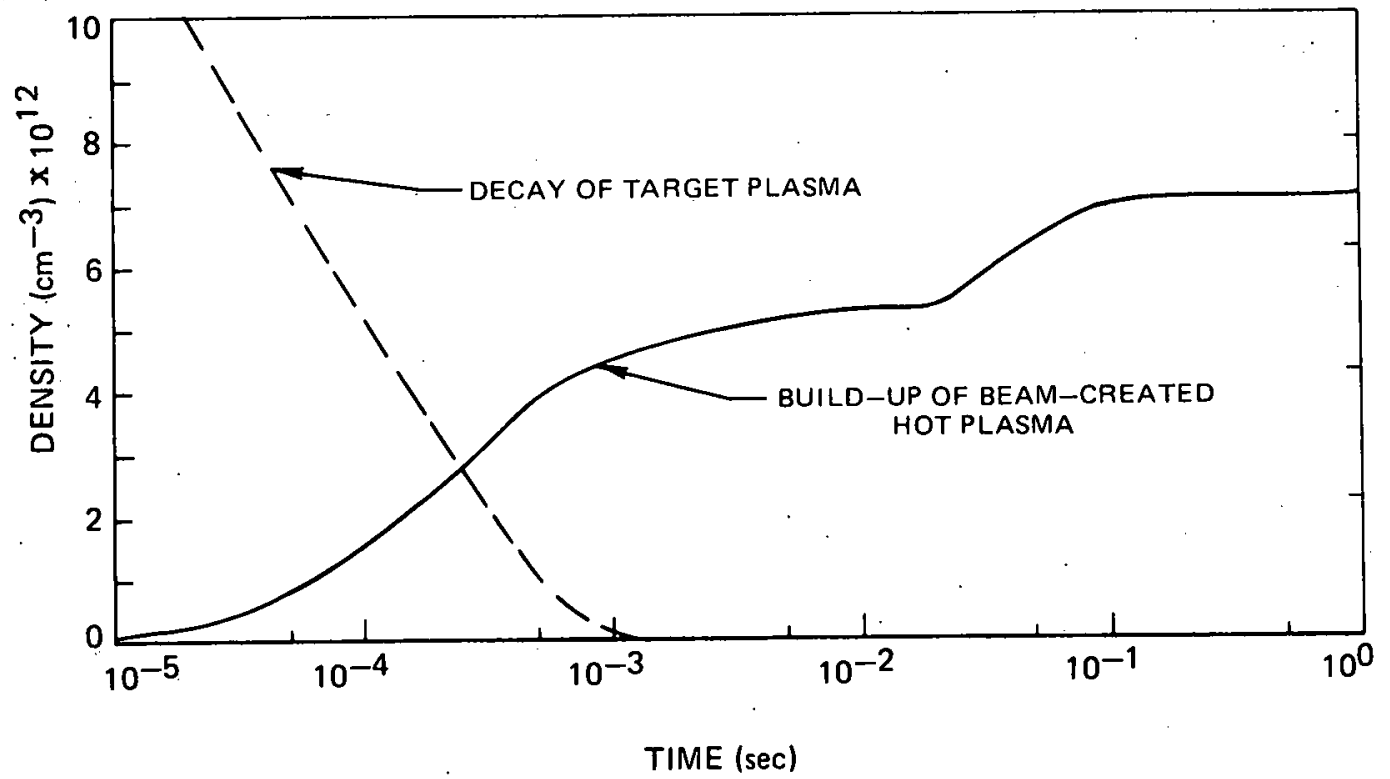




\section{SUSTENANCE IN HIGH VACUUM}
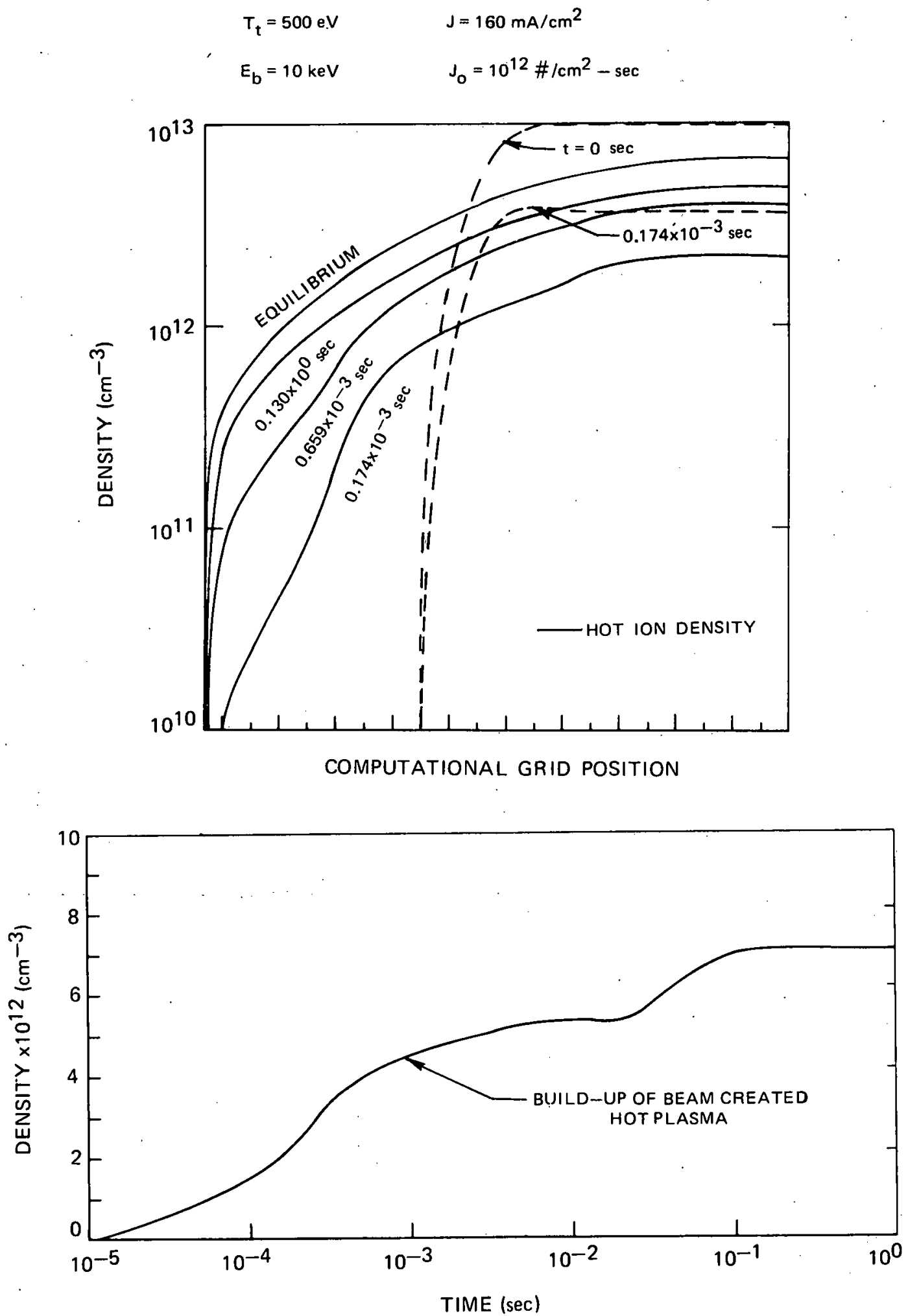


\section{INJECTION INTO TARGET PLASMA IN POOR VACUUM}
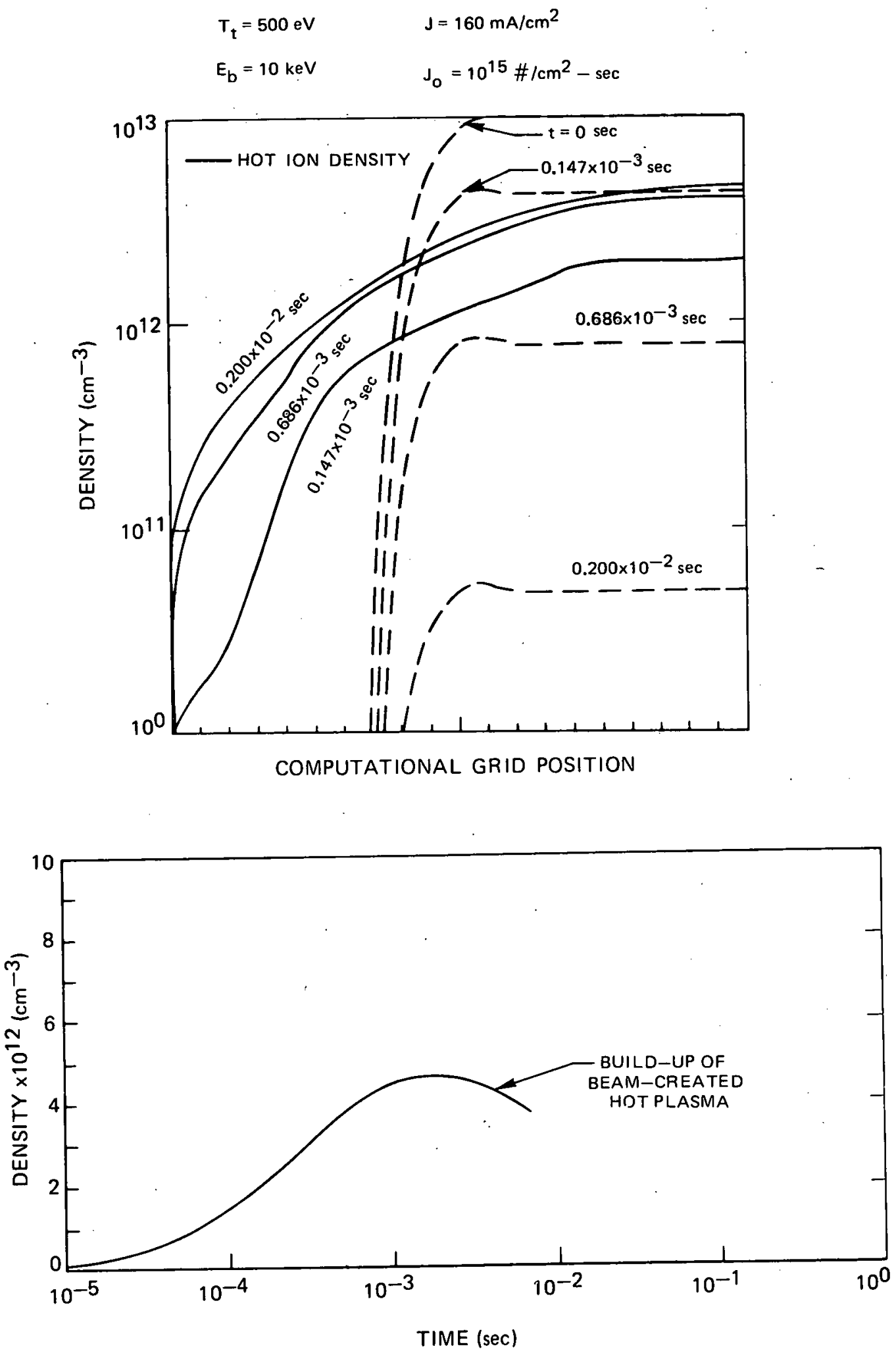